\title{
The inner structure of early-type galaxies in the Illustris simulation
}

\author{
Dandan $\mathrm{Xu}^{1 \star}$, Volker Springel ${ }^{1,2}$, Dominique Sluse $^{3}$, Peter Schneider ${ }^{4}$ \\ Alessandro Sonnenfeld ${ }^{5}$, Dylan Nelson ${ }^{6}$, Mark Vogelsberger ${ }^{7}$, Lars Hernquist ${ }^{8}$ \\ 1 Heidelberg Institute for Theoretical Studies, Schloss-Wolfsbrunnenweg 35, 69118 Heidelberg, Germany \\ 2 Zentrum für Astronomie der Universität Heidelberg, Astronomisches Recheninstitut, Mönchhofstr. 12-14, 69120 Heidelberg, Germany \\ 3 STAR Institute, Quartier Agora - Allée du six Août, 19c B-4000 Liège, Belgium \\ 4 Argelander-Institut für Astronomie, Universität Bonn, Auf dem Hügel 71, 53121 Bonn, Germany \\ 5 Kavli Institute for the Physics and Mathematics of the Universe of Tokyo, 5-1-5 Kashiwanoha Kashiwa, 277-8583 Japan \\ 6 Max Planck Institute for Astrophysics, Karl-Schwarzschild-Str. 1, Postfach 1317, D-85741 Garching, Germany \\ 7 Department of Physics, Massachusetts Institute of Technology, 77 Massachusetts Avenue, Cambridge, United States \\ 8 Harvard Astronomy Department, 60 Garden Street MS 46, Cambridge, MA 02138, United States
}

12 April 2017

\begin{abstract}
Early-type galaxies provide unique tests for the predictions of the cold dark matter cosmology and the baryonic physics assumptions entering models for galaxy formation. In this work, we use the Illustris simulation to study correlations of three main properties of early-type galaxies, namely, the stellar orbital anisotropies, the central dark matter fractions and the central radial density slopes, as well as their redshift evolution since $z=1.0$. We find that lower-mass galaxies or galaxies at higher redshift tend to be bluer in rest-frame colour, have higher central gas fractions, and feature more tangentially anisotropic orbits and steeper central density slopes than their higher-mass or lowerredshift counterparts, respectively. The projected central dark matter fraction within the effective radius shows a very mild mass dependence but positively correlates with galaxy effective radii due to the aperture effect. The central density slopes obtained by combining strong lensing measurements with single aperture kinematics are found to differ from the true density slopes. We identify systematic biases in this measurement to be due to two common modelling assumptions, isotropic stellar orbital distributions and power-law density profiles. We also compare the properties of early-type galaxies in Illustris to those from existing galaxy and strong lensing surveys, we find in general broad agreement but also some tension, which poses a potential challenge to the stellar formation and feedback models adopted by the simulation.
\end{abstract}

Key words: gravitational lensing: strong - galaxies: haloes - galaxies: structure cosmology: theory - dark matter.

\section{INTRODUCTION}

Cosmic structures grow in a hierarchical fashion whereby small halos merge to form larger ones. This prevailing picture of structure assembly is predicted by the cold dark matter (CDM) cosmological theory. Early-type galaxies are in some sense the end products of the corresponding galaxy merging and accretion processes (e.g., White \& Rees 1978; Davis et al. 1985; Kauffmann et al. 1993; Cole et al. 1994; Kauffmann 1996), and thus provide an interesting testing ground of the CDM cosmological model.
Due to their association with mergers, early-type galaxies tend to live in high-density environments and have quite old stellar populations. Though traditionally thought to be structureless and "red and dead", mounting evidence has shown over the past decades that there is considerable richness and complexity in the origin and evolution of their mass-size relations, star formation activities, and central density profiles, etc. Utilizing for example their fundamental plane relations and/or their gravitational lensing effects, early-type galaxies are also widely used as probes of the high-redshift Universe, making them a powerful and important tool in modern astrophysics and cosmology. 
their central dark matter fraction and central density profiles are particularly closely tied to their formation and evolution histories. The CDM model predicts universal NFW (Navarro et al. 1997) profiles for the dark matter distribution in halos over a wide range of mass scales. However, on galaxy-scales, baryonic matter strongly dominates the central regions of dark matter halos. As a result of dissipation, baryons follow more centrally concentrated density distributions, which in turn changes the central mass fraction of dark matter (e.g., Treu \& Koopmans 2004; Koopmans et al. 2006; Napolitano et al. 2010; Barnabè et al. 2011; Ruff et al. 2011; Cappellari et al. 2013; Sonnenfeld et al. 2015; Oguri et al. 2014) and also modifies the inner dark matter slopes, making them steeper than the NFW prediction (e.g., Sonnenfeld et al. 2012; Grillo 2012; Johansson et al. 2012; Remus et al. 2013; Cappellari et al. 2013; Oguri et al. 2014).

Regarding the total density profiles in the central regions, the most intriguing fact is that the sum of dark and baryonic matter approximately follows an isothermal profile, i.e. $\rho(r) \propto r^{-2}$, even though neither the dark nor the baryonic matter exhibit an isothermal distribution individually. Evidence for such a profile comes from stellar kinematical studies (e.g., Binney \& Tremaine 2008; Cappellari et al. 2015), strong and weak lensing observations (e.g., Rusin et al. 2003; Koopmans et al. 2006, 2009; Gavazzi et al. 2007; Barnabè et al. 2009, 2011; Auger et al. 2010b; Ruff et al. 2011; Bolton et al. 2012; Sonnenfeld et al. 2013), as well as X-ray studies of early-type galaxies (e.g., Humphrev et al. 2006; Humphrev \& Buote 2010). Theoretically, the formation of such a total density distribution is speculated to occur through a two-phase process, where active (central) star formation and adiabatic contraction in an early stage is followed by dissipationless mergers and accretion later on. The former steepens the central density slopes while the latter in general makes them shallower. The observed central density slopes and their evolutionary trend, therefore, put stringent constraints on both the CDM structure formation model and models for baryonic physics processes.

Galaxy-scale strong gravitational lensing is among the major tools to probe galaxies out to high redshifts. It robustly measures the projected mass within the Einstein radius, typically at within a few kpc from the centre of a lensing galaxy. Traditionally, the strong lensing technique has also been combined with stellar kinematics, which provides the mass measured within some different aperture radius (Romanowsky \& Kochanek 1999). The combination thus allows measurements of the radial density slopes and dark matter fractions. The method has been put into good use for many existing strong lensing surveys, e.g., the Lenses Structure and Dynamics Survey (LSD; e.g., Treu \& Koopmans 2004); the Sloan Lens ACS Survey (SLACS; Koopmans et al. 2006, 2009; Bolton et al. 2008a; Auger et al. 2010b), the BOSS Emission-Line Lens Survey (BELLS; Brownstein et al. 2012; Bolton et al. 2012), and the Strong Lensing Legacy Survey (SL2S; Ruff et al. 2011; Gavazzi et al. 2012; Sonnenfeld et al. 2013, 2015). To date, hundreds of strong-lensing early-type galaxies have been well studied out to redshift $z=1.0$. The average central density slopes have been found to be approximately consistent with isothermal radial profiles with a small intrinsic scatter. This isothermal behaviour seems to have evolved very little in the past 7 Gyrs (i.e. since $z=1.0$ ).

To date various theoretical approaches, including semianalytical models and N-body simulations have been exploited in order to address open issues regarding the masssize relations, the central dark matter fractions, as well as the total density slopes of early-type galaxies (e.g., Nipoti et al. 2009a, b; Johansson et al. 2012; Remus et al. 2013; Dubois et al. 2013; Sonnenfeld et al. 2014). These studies have made important progress in finding the missing links in the framework of the formation and evolution theories and in understanding potential systematic biases of the observational techniques. However, these modelling techniques often lacked a self-consistent treatment of baryonic physics in a cosmological context, which limited their predictive power.

In this regard, the latest generation of cosmological hydrodynamical simulations of galaxy formation represents a significant step forward and enables a much closer comparison between theoretical predictions and observations (e.g., Wellons et al. 2015, 2016; Remus et al. 2016). Among these new simulations is the Illustris Project (Vogelsberger et al. 2014a, b; Genel et al. 2014; Sijacki et al. 2015; Nelson et al. 2015), which provides an ideal tool for such purposes. Run with the accurate moving-mesh hydro solver AREPO (Springel 2010), the Illustris simulation took into account a wide range of baryonic processes, resolved the formation of 40000 galaxies of different morphology types, and managed to reproduce many fundamental properties of observed galaxies.

In this paper, we report a variety of properties of earlytype galaxies in the highest resolution simulation of the Illustris project 1 In particular, we investigate the dependencies and the redshift evolution since $z=1.0$ of (1) the stellar orbital anisotropies, (2) the central dark matter mass fractions, and (3) the central radial density slopes over the past $\sim 7$ Gyrs. The main aim of this work is to unveil correlations between these galaxy properties and to link them to underlying physical processes. Also, we are interested in identifying differences between simulation predictions and observations, through which one can establish systematic biases of observational techniques and the interpretations of the measurements.

The paper is organized as follows. In Sect.2, we describe in detail how the light distributions of the simulated galaxies were determined and how the observed galaxy properties were measured. In Sect. 3, we report general properties of the selected galaxies, including galaxy and total matter morphologies (Sect.3.1), the mass-size-velocity dispersion relations (Sect. 3.2), and fundamental plane properties (Sect.3.3). We then present the dependencies and the redshift evolution of the stellar orbital anisotropies in Sect. 4, those of the central dark matter fraction in Sect. 5 and those of the central density slopes in Sect.6. Finally, a discussion and our conclusions are given in Sect.7. We note that

1 The highest resolution Illustris run covers a cosmological volume of $(106.5 \mathrm{Mpc})^{3}$ and has a dark matter mass resolution of $6.26 \times 10^{6} \mathrm{M}_{\odot}$ and an initial baryonic mass resolution of $1.26 \times 10^{6} \mathrm{M}_{\odot}$, resolving gravitational dynamics down to a physical scale of $\epsilon=710 \mathrm{pc}$. 
all the galaxy properties reported in this paper have been made publicly available from the Illustris website. In the Appendix, we give a brief summary of the content of this online catalogue.

In this work, we adopted the same cosmology as used in the Illustris simulation, i.e., a matter density of $\Omega_{\mathrm{m}}=0.27$, a cosmological constant of $\Omega_{\Lambda}=0.73$, a Hubble constant $h=H_{0} /\left(100 \mathrm{~km} \mathrm{~s}^{-1} \mathrm{Mpc}^{-1}\right)=0.70$ and a linear fluctuation amplitude $\sigma_{8}=0.81$. These values are consistent with the Wilkinson Microwave Anisotropy Probe (WMAP)-9 measurements (Hinshaw et al. 2013).

\section{ILLUMINATING GALAXIES AND MEASURING GALAXY PROPERTIES}

The galaxies simulated in Illustris are identified as gravitationally bound structures of gas cells, dark-matter particles, and stellar particles using the SUBFIND algorithm (Springel et al. 2001; Dolag et al. 2009). In this section, we describe how we calculated in a post-processing procedure observational properties for the simulated galaxies.

It is noteworthy to recall that when a smaller halo is accreted onto a bigger structure, its dark and baryonic matter at the outskirt can be tidally stripped while sinking to the centre of the host. As a result, a (galaxy) halo in a group or cluster environment can be composed of a tightly-bound central region and a loosely-bound outskirt that extends to large radii. Observationally, the measured "galaxy" properties are only accounting for the former. However, the latter component, which in the context of galaxy clusters is also known as "intracluster light", can make up for $\leqslant 50 \%$ of the total stellar mass and luminosity (e.g., Lin \& Mohr 2004; Zibetti et al. 2005; Puchwein et al. 2010). This stellar mass is still bound gravitationally to the galaxy and hence normally included in the raw measurement of SUBFIND.

Often, a simple radial cut-off radius has been used in numerical simulations to deal with this problem and to calculate properties that are associated with the tightly-bound galaxy component. For example, in Puchwein et al. (2010, see their eq.1), a halo mass-dependent radial cut was applied to the central brightest cluster galaxies (BCGs) more massive than $2 \times 10^{13} M_{\odot}$. Schaye et al. (2015) applied a three-dimensional (3-D) radial cut of 30 physical kpc to all galaxies from the EAGLE project. Such a choice was found to significantly affect the measured properties of the tightlybound galaxy component that has a stellar mass more than $10^{11} M_{\odot}$. Such a radial cut was also able to reproduce the observed galaxy stellar mass function that was derived using the frequently applied Petrosian apertures (see $§ 5.1 .1$ in Schaye et al. (2015) for detailed discussion). In this work, we used a similar strategy. In calculations of projectionindependent intrinsic galaxy properties, such as the stellar mass, a $3-\mathrm{D}$ radial cut of $30 \mathrm{kpc}$ is adopted. For the luminosity-based and thus projection-dependent properties, we used a 2-D radial cut of $30 \mathrm{kpc}$ from the centre of light for a given galaxy projection. Any resolution element still bound to the system but located or projected outside this radius is excluded from the corresponding calculations.

\subsection{From stellar particles to galaxy light}

We calculated the emission properties of individual simulated galaxies from the luminosities of their constituent stellar particles. Each stellar particle of $\sim 10^{6} h^{-1} \mathrm{M}_{\odot}$ is treated as a coeval single stellar population that has the Chabrier (2003) initial mass function (IMF). Given the star formation time and metallicity of each stellar particle, a "raw" luminosity $L_{\text {raw }}$ in a given bandpass can thus be derived using the Bruzual \& Charlot (2003) stellar population synthesis (SPS) model GALAXEV.

This raw galaxy light can be processed by dust through absorption and scattering at shorter wavelengths and reemission at longer wavelengths. We implemented such a dust attenuation process through a simple semi-analytical approach as follows. Assuming that a galaxy can be approximated as a uniformly mixed slab of stars, gas and dust, the amount of extinction of each stellar particle is given by

$$
\frac{L_{\mathrm{obs}}}{L_{\mathrm{raw}}}=\frac{1-\exp \left(-\tau_{\lambda}\right)}{\tau_{\lambda}},
$$

where $L_{\text {obs }}$ and $L_{\text {raw }}$ are the "observed" (dust-attenuated) and the raw (dust-free) luminosities, respectively. $\tau_{\lambda}$ is the optical depth, which depends on the wavelength $\lambda$ and is caused by both dust absorption and scattering along the line of sight.

In order to predict the amount of dust extinction for each stellar particle, a $100 \times 100$ mesh that covers the (projected) central region of a simulated galaxy, from -3 to +3 times the half-stellar mass radius in either dimension, was used to tabulate the distribution of $\tau_{\lambda}$. The $\tau_{\lambda}$-mesh can be derived from the neutral hydrogen (HI) distribution of the gas cells using a semi-analytical prescription as follows.

A redshift- and metallicity-dependent optical depth $\tau_{\lambda}^{\mathrm{a}}$ due to the dust absorption process is traditionally modelled as (e.g., Guiderdoni \& Rocca-Volmerange 1987; Devriendt et al. 1999; Devriendt \& Guiderdoni 2000)

$$
\tau_{\lambda}^{\mathrm{a}}=\left(\frac{A_{\lambda}}{A_{\mathrm{v}}}\right)_{Z_{\odot}}(1+z)^{\beta}\left(\frac{Z_{\mathrm{g}}}{Z_{\odot}}\right)^{s} \frac{\left\langle N_{\mathrm{H}}\right\rangle}{2.1 \times 10^{21} \mathrm{~cm}^{-2}},
$$

where $\left(A_{\lambda} / A_{\mathrm{V}}\right)_{Z_{\odot}}$ is the solar-neighbourhood extinction curve (Cardelli et al. 1989), $Z_{\mathrm{g}}$ is the gas metallicity (of the galaxy) and $Z_{\odot}=0.02$ is the measured value for the Sun. $\left\langle N_{\mathrm{H}}\right\rangle$ is the average neutral hydrogen column density. A power-law index $s=1.35$ for $\lambda<$ $2000 \AA$ and $s=1.6$ for $\lambda>2000 \AA$ was found by Guiderdoni \& Rocca-Volmerange (1987) for the metallicity dependence. In addition, Kitzbichler \& White (2007) found that $\beta=-0.5$ can reproduce measurements of Lyman-break galaxies at $z \sim 3$. We also adopted this value for $\beta$.

To also account for dust scattering, we used an approximate solution from Calzetti et al. (1994), in which the total effective optical depth $\tau_{\lambda}$ is given by

$$
\tau_{\lambda}=h_{\lambda} \sqrt{1-\omega_{\lambda}} \tau_{\lambda}^{\mathrm{a}}+\left(1-h_{\lambda}\right)\left(1-\omega_{\lambda}\right) \tau_{\lambda}^{\mathrm{a}},
$$

where $\omega_{\lambda}$ is the albedo, defined as the ratio between the scattering and the extinction coefficients, and $h_{\lambda}$ and $1-h_{\lambda}$ are the weighing factors for the isotropic and the forwardonly scattering, respectively.

For each cell $(i, j)$ of the $\tau_{\lambda}$-mesh, a mean neutral hydrogen column density $\left\langle N_{\mathrm{H}}^{(i, j)}\right\rangle$ was first calculated by scattering the (fractional) cold hydrogen masses of each gas cell 
onto the mesh using a SPH smoothing technique. $\left\langle N_{\mathrm{H}}^{(i, j)}\right\rangle$ was then converted into $\tau_{\lambda}^{(i, j)}$ via Eqs. (2) and (3), assuming $\lambda$ being the effective wavelength of a given bandpass. For stellar particles that are projected within the mesh coverage, the exact $\tau_{\lambda}$ (at the position of a given stellar particle) was then interpolated from the $\tau_{\lambda}$-mesh. For those outside, $\tau_{\lambda}=0$ was assumed. The total light distribution of a simulated galaxy in its viewing direction in a given bandpass was then computed from $L_{\text {obs }}$, the "observed" (dust-attenuated) luminosities of the constituent stellar particles.

\subsection{Measurement of galaxy centres, ellipticities and orientation angles}

Each simulated galaxy (together with its dark matter halo) has a "centre" that was calculated as the position of the particle with the minimum gravitational potential found using SubFind. The observed galaxy centre is lightbased and projection-dependent, we therefore defined a twodimensional $(2-\mathrm{D})$ galaxy centre $\left(x_{\mathrm{gc}}, y_{\mathrm{gc}}\right)$ as:

$$
\begin{aligned}
& x_{\mathrm{gc}}=\sum_{i} L_{i} x_{i}\left(\sum_{i} L_{i}\right)^{-1}, \\
& y_{\mathrm{gc}}=\sum_{i} L_{i} y_{i}\left(\sum_{i} L_{i}\right)^{-1},
\end{aligned}
$$

where $x_{i}$ and $y_{i}$ are the $x$ - and $y$-coordinates of the $i$-th stellar particle in the plane of a given galaxy projection. $L_{i}$ is the "observed" (dust-attenuated) luminosity, and $\sum_{i} L_{i}$ is the total luminosity within a given aperture.

The orientation and ellipticity (or axis ratio) of a galaxy were measured through luminosity-weighted second moments, which are defined as:

$$
\begin{aligned}
& M_{x x}=\sum_{i} L_{i}\left(x_{i}-x_{\mathrm{gc}}\right)^{2}\left(\sum_{i} L_{i}\right)^{-1}, \\
& M_{y y}=\sum_{i} L_{i}\left(y_{i}-y_{\mathrm{gc}}\right)^{2}\left(\sum_{i} L_{i}\right)^{-1}, \\
& M_{x y}=\sum_{i} L_{i}\left(x_{i}-x_{\mathrm{gc}}\right)\left(y_{i}-y_{\mathrm{gc}}\right)\left(\sum_{i} L_{i}\right)^{-1} .
\end{aligned}
$$

The axis ratio $b / a$ of a galaxy projection is then given by:

$$
b / a=\left(\frac{M_{x x}+M_{y y}-\sqrt{\left(M_{x x}-M_{y y}\right)^{2}+4 M_{x y}^{2}}}{M_{x x}+M_{y y}+\sqrt{\left(M_{x x}-M_{y y}\right)^{2}+4 M_{x y}^{2}}}\right)^{1 / 2} .
$$

The orientation angle $\phi_{\mathrm{PA}}$ is given by:

$$
\phi_{\mathrm{PA}}=\frac{1}{2} \tan ^{-1}\left(\frac{2 M_{x y}}{M_{x x}-M_{y y}}\right) .
$$

\subsection{Luminosity and effective radius measurement}

In order to calculate the galaxy luminosity and effective radius within non-circular apertures, we first assumed that the 2-D surface brightness distribution in a given viewing projection follows a series of elliptical isophotes that are well described by $b / a$ and $\phi_{\mathrm{PA}}$ measured using Eqs. (6) and (7) within three-times the half stellar-mass radius from the centre of the galaxy. A "summed" galaxy luminosity $L^{\text {sum }}$ is calculated by directly summing up $L_{\text {obs }}$ for all constituent stellar particles that are projected within $30 \mathrm{kpc}$.

A "direct" effective radius $R_{\mathrm{eff}}^{\mathrm{dir}}$ is determined as the geometric mean of the semi-major and semi-minor radii of the elliptical isophote which encloses half of $L^{\text {sum }}$. We used $L^{\text {sum }}$ and $R_{\text {eff }}^{\text {dir }}$ as approximate estimates of the intrinsic luminosity and size of a simulated galaxy. They are, however, different from those used in observations. In order to make a fair comparison, we followed one of the observational conventions to derive a "model" luminosity $L^{\text {mod }}$ and a "model" effective radius $R_{\text {eff }}^{\text {mod }}$, i.e., by fitting a Sersic profile (Sérsic 1963) to the radial distribution of the elliptical isophotes that are assumed to closely trace the 2-D surface brightness distribution of a given galaxy projection.

The surface brightness $I(R)$ of a Sersic profile at radius $R$ is given by:

$$
I(R)=I\left(R_{\text {eff }}\right) \exp \left\{-b_{m}\left[\left(R / R_{\text {eff }}\right)^{1 / m}-1\right]\right\},
$$

where $R_{\text {eff }}$ is the effective radius of the Sersic profile, and $m$ is the Sersic index. The factor $b_{m}$ can be determined by satisfying $\int_{0}^{R_{\text {eff }}} I(R) R \mathrm{~d} R=\frac{1}{2} \int_{0}^{\infty} I(R) R \mathrm{~d} R$. We adopted the values of $b_{m}$ from Ciotti \& Bertin (1999) for $m \geqslant 0.36$ and those of MacArthur et al. (2003) for $m<0.36$. The luminosity $L(R)$ of a Sersic distribution enclosed within a radius of $R$ is given by:

$$
L(R)=I\left(R_{\mathrm{eff}}\right) \exp \left(b_{m}\right) R_{\mathrm{eff}}^{2} \frac{2 \pi m}{b_{m}^{2 m}} \gamma\left(2 m, b_{m}\left(R / R_{\mathrm{eff}}\right)^{1 / m}\right),
$$

where $\gamma(a, x)$ is the incomplete gamma function, $\gamma(a, x) \equiv$ $\int_{0}^{x} \exp (-t) t^{a-1} \mathrm{~d} t$. Note that in the formulae above, the radius $R$ is defined as the geometric mean of the semi-major and semi-minor radii of the elliptical isophotes.

For each galaxy projection, the binned radial profile between $0.05 R_{\text {eff }}^{\text {dir }}$ and $3.0 R_{\text {eff }}^{\text {dir }}$ was fitted by the Sersic model using a minimum $\chi^{2}$ fitting approach. In general, the radial surface brightness distributions of the simulated galaxies well follow Sersic profiles. The model effective radius $R_{\text {eff }}^{\bmod }$ of a given galaxy projection was set to be the best-fitting Sersic effective radius. The model luminosity $L^{\text {mod }}$ was then given by the best-fitting Sersic luminosity within $7 R_{\mathrm{eff}}^{\mathrm{mod}}$.

We measured the light distribution of each simulated galaxy in a variety of optical filter bandpasses in order to compare with latest observations. Note that galaxy luminosities (magnitudes) and effective radii are band-dependent (also see La Barbera \& de Carvalho 2009). Hereafter, the effective radii $R_{\text {eff }}$ specifically refer to the Sersic model effective radius that was derived in the rest-frame Johnson $V$-band. For galaxies at $z=0.3$ measured in this band, the luminosity ratios have a (stellar-mass weighted) mean of $\left\langle L^{\mathrm{mod}} / L^{\text {sum }}\right\rangle=1.06$ with a standard deviation of 0.16 , and the effective radii ratios have a (stellar-mass weighted) mean of $\left\langle R_{\text {eff }}^{\text {mod }} / R_{\text {eff }}^{\text {dir }}\right\rangle=1.13$ with a 0.33 scatter. These ratios vary little across the investigated redshift range.

In Fig. 1 we present the dust-corrected luminosity functions (LF) of the Illustris galaxies at intermediate redshifts $(0.2<z<0.4)$, measured in the Johnson- $B$ band. Plotted in the same panel are the Schechter function fits to the observed LFs from DEEP2 and COMBO-17 surveys (Faber et al. 2007). The red and blue galaxy samples were selected from the simulation according to eq. 1 in Faber et al. (2007). The Illustris galaxy LFs have been found to roughly match observational results in various bands and within a wide range of redshifts (also see Vogelsberger et al. 2014b; Hilbert et al. 2016). We note, in particular, that the effect of dust attenuation is significant especially for blue 


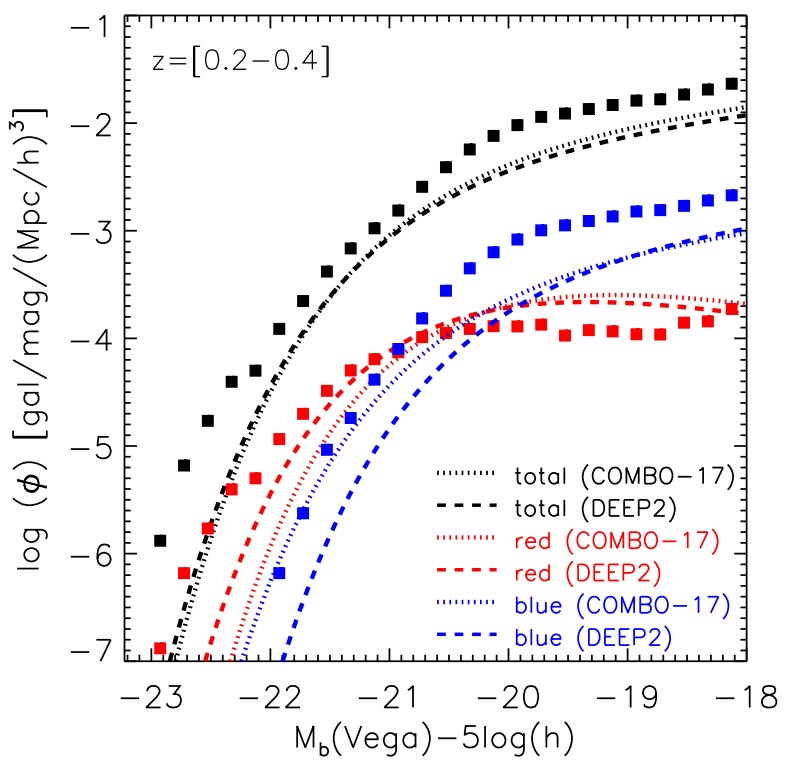

Figure 1. Galaxy luminosity functions at intermediate redshifts $(0.2<z<0.4)$, measured in Johnson- $B$ band. Simulation data are given by square symbols, where black, red and blue colours represent the total, "red", and "blue" galaxy samples, respectively. The latter two are rescaled by a factor of 0.1 for graphical clarity. The red and blue galaxy samples were selected from the simulation according to eq. 1 in Faber et al. (2007). The dotted and the dashed lines give single power-law Schechter function fits to the observed galaxy luminosity functions from the COMBO-17 and DEEP2 surveys, respectively.

galaxy sample, which, without taking dust into account, would contribute to at least half of the total galaxy count at any given luminosity up to $M \sim-22$. In comparison, the observed LFs have this transition at two magnitudes higher, where the blue and red galaxy samples have equal contributions to the total LF and below which (in the brighter end), red galaxies would dominate the total LF. As can be seen, applying dust attenuation to the simulated galaxies has pushed this transition magnitude much closer to the observation. At the bright end, there are larger measurement uncertainties, and the derived magnitudes can differ strongly depending on the assumed light profiles (e.g., Bernardi et al. 2013).

\subsection{Galaxy type classification}

The method that we used for galaxy classification is similar to the practice of the Sloan Digital Sky Survey (SDSS). Using the rest-frame SDSS $g, r$ and $i$ filters simultaneously, we fitted both de Vaucouleurs profiles (de Vaucouleurs 1948) and exponential profiles to the radial surface brightness distributions of the elliptical isophotes (between $0.05 R_{\text {eff }}^{\text {dir }}$ to $\left.3.0 R_{\text {eff }}^{\text {dir }}\right)$. If the former provides a better fit, then the galaxy is classified as an early-type (elliptical) galaxy, whereas if the latter fits better, then it is considered a late-type (disk) galaxy.

Fig. 2] shows histograms of the best-fitting Sersic indices of the simulated early- and late-type galaxies at redshift $z=0.3$. As expected, the former have larger $(m \gtrsim 2)$ Ser-

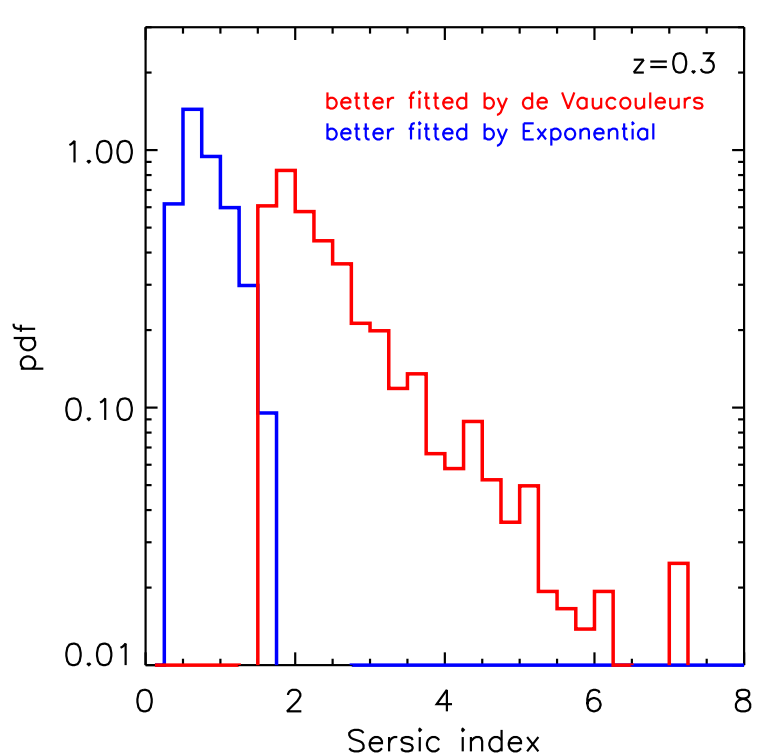

Figure 2. Histograms of the best-fitting Sersic indices of galaxies whose radial surface brightness distributions are better fitted by de Vaucouleurs profiles (red) and of those whose radial distributions are better fitted by exponential profiles (blue).

sic indices, while the latter have $m<2$. As an illustration, we select two typical galaxies with different type classifications and show their synthesized light distributions in the top panel of Fig. 3, where the left and right sub-panels display an elliptical and a disk galaxy at $z=0.3$, respectively. The image was made by combining the surface brightness distributions in the rest-frame SDSS $g, r$ and $i$ filter bandpasses, within a $3 R_{\text {eff }} \times 3 R_{\text {eff }}$ region from the light centres of the corresponding galaxy projections.

The lower panels of Fig. 3 show the radial surface brightness distributions of the early- (middle) and late-type (bottom) galaxies above. In these panels, black dots are the binned data measured in the SDSS $r$ band, blue lines give the best-fitting Sersic profiles (Sersic indices of 2.35 and 1.27, respectively), while red lines show the best-fitting de Vaucouleurs and exponential profiles for the two galaxies, respectively.

\section{GENERAL PROPERTIES OF THE GALAXY CATALOGUE}

Apart from the above mentioned luminous properties, we derived a wide range of projection-dependent lensing and dynamical properties, such as the Einstein radii $R_{\mathrm{E}}$, dark matter fractions $f_{\mathrm{dm}}$, stellar velocity dispersions $\sigma$, orbital anisotropies $\beta$, and various mass density slope estimators $\gamma$. All these calculations were carried out for galaxies (regardless of galaxy types) with stellar masses $M_{*} \gtrsim 10^{10} \mathrm{M}_{\odot}$ (corresponding to more than $\sim 10000$ stellar particles) at redshifts in the range $z \in[0.1,1.0]$ with an interval spacing $\Delta z=0.1$. We defined artificial source redshifts at $z_{\mathrm{s}}=\left[\begin{array}{llllllll}0.5, & 0.6 & 0.7, & 0.9, & 2.0 & 2.0 & 2.0 & 2.0,2.0,2.0\end{array}\right] \mathrm{ac}-$ 

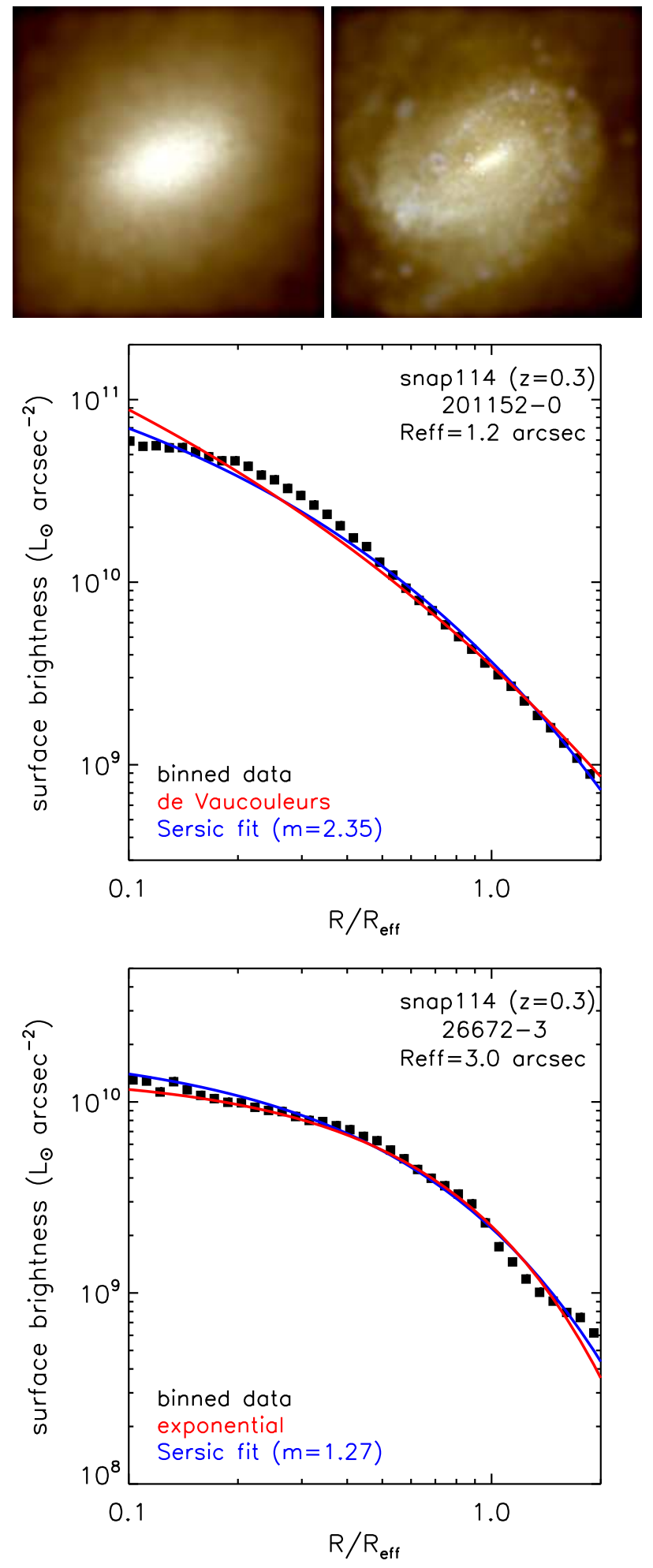

Figure 3. The top row presents synthesized images of an elliptical (left panel) and a disk (right panel) galaxy from the simulation at redshift $z=0.3$. The image was made by combining the surface brightness distributions in the rest-frame SDSS $g, r$ and $i$ filter bandpasses, within a $3 R_{\text {eff }} \times 3 R_{\text {eff }}$ region from the galaxy centres. The middle and bottom panels show radial surface brightness distributions of the early- and late-type galaxies, respectively. In these panels, black dots are the binned data measured in the SDSS $r$ band, blue lines give the best-fitting Sersic profiles (Sersic indices of 2.35 and 1.27 , respectively), and red lines show the bestfitting de Vaucouleurs and exponential profiles of the two galaxies, cordingly, in order to calculate the Einstein radi:2. These choices for $z_{\mathrm{s}}$ are motived by the observed lens-source redshift distributions. All the calculated properties have been catalogued and are publicly available from the Illustris website (www.illustris-project.org), and a detailed description of the different catalogue fields can be found in the Appendix.

In the following part of the paper, we aim at presenting the statistical properties of the simulated early-type galaxies and comparing them to those resulted from the SDSS early-type galaxy survey (Hyde \& Bernardi 2009a), and to those from recent strong lensing surveys, i.e., the SLACS and SL2S surveys, as well as those from the Cosmological Monitoring of Gravitational Lenses projects (COSMOGRAIL; Sluse et al. 2012). These surveys provide comprehensive observational samples predominantly composed of isolated early-type galaxies within a wide redshift range, similar to the one studied here.

We are aware that the lensing selection effect has always been a complication when comparing simulation samples to observations (or using observed samples to interpret physical properties of galaxies). Detailed work in this regard can be found in, e.g., Mandelbaum et al. (2009). For the present study, we do not apply any sophisticated selection criteria. We selected Illustris galaxies at each given redshift according to the following two simple criteria: (1) central and early-type galaxies, and (2) the stellar line-of-sight velocity dispersions $\sigma_{e / 2}$ (measured within $0.5 R_{\text {eff }}$ ) satisfies $\sigma_{e / 2} \in[160,400] \mathrm{km} \mathrm{s}^{-1}$. These criteria are mainly observationally motivated by the strong lensing galaxy surveys. As will be seen in Sect.3.2, the criteria above resulted in galaxy samples that roughly reproduced the observed masssize-velocity dispersion relations.

It is worth noting that when calculating properties of a central galaxy, any self-gravitationally-bound substructures (satellite galaxies) identified by SUBFIND were excluded. To increase the sample size, we treated galaxies that are viewed along the three principal directions of the simulation box as independent. The selection criteria resulted in $\sim 600$ independent galaxy projections at each of the simulation redshifts investigated. In this section, we present the measured shapes of the luminous and dark matter distributions (Sect. 3.1), mass-size-velocity dispersion relations (Sect. 3.2) and fundamental plane relations (Sect.3.3) of the selected galaxy samples at various redshifts.

\subsection{Shape of luminous and total matter}

It has always been a critical question "how well the light follows the matter". In central regions of early-type galaxies, the projected total matter distributions are in general rounder 3 than the luminous (stellar) distributions. This can

2 The Einstein radius $R_{\mathrm{E}}$ is found as the radius within which the mean surface density is equal to the lensing critical density $\Sigma_{\mathrm{cr}}=\left(\frac{c^{2}}{4 \pi G}\right)\left(\frac{D_{\mathrm{s}}}{D_{\mathrm{ds}} D_{\mathrm{d}}}\right)$, where $D_{\mathrm{d}}, D_{\mathrm{s}}$ and $D_{\mathrm{ds}}$ are the angular diameter distances to the lens, to the source, and from the lens to the source, respectively.

3 The situation is the opposite at larger radii for which weak lensing technique can be applied. At those radii, dark matter dominates the total matter distribution, which appears to be flatter than the light profile (e.g., Hoekstra et al. 2004). 
be seen from the top panel of Fig. 4 which shows the ratio between the luminous axis ratio $(b / a)_{\text {gal }}$ and the total axis ratio $(b / a)_{\text {tot }}$, as a function of the central stellar velocity dispersion $\sigma_{e / 2}$ : the solid blue and red line indicates the median of the distribution measured within $0.5 R_{\text {eff }}$ and $2.0 R_{\text {eff }}$, respectively; the dashed lines show the $90 \%$ boundaries of the distributions. Note that the ellipticity ratio decreases as the aperture size increases from $0.5 R_{\text {eff }}$ to $2.0 R_{\text {eff }}$. This is mainly attributed to the fact that as the aperture size increases the total matter distribution becomes rounder, while the ellipticity of the stellar distribution only varies mildly. The ellipticity ratio distribution does not seem to evolve strongly with redshift, at higher redshift the scatter increases marginally.

Observationally, the shape of the total matter distribution of a (lensing) galaxy can be inferred via a lens modelling technique, while that of the stellar distribution can be obtained from direct imaging. In this regard, previous studies based on singular isothermal ellipsoidal (SIE) lens models found that the median ellipticity ratios of the galaxy samples from the SLACS, SL2S and COSMOGRAIL (see Koopmans et al. 2006; Gavazzi et al. 2012; Sluse et al. 2012; Shu et al. 2015) are very close to 1.0, which lies above both the median and the $90 \%$ upper boundary of the simulation distribution. Regarding this disagreement, we note that, as will be seen in Sect. 5, the central dark matter fractions in the Illustris early-type galaxies were found to be systematically higher compared to the observational results. This may also explain the systematically lower ellipticity ratios between the luminous and the total matter distributions of the simulated galaxies. However, it is also noteworthy to point out that recent lensing studies that have either applied non-parametric lens modelling (e.g., Bruderer et al. 2016) or adopted novel techniques to extract galaxy morphology data from adaptive optics observations (e.g., Rusu et al. 2016) found that the total matter distributions are systematically rounder than the stellar distributions in central regions of early-type galaxies, in agreement with our simulation results.

The orientation of the projected total matter distribution follows in general that of the light distribution very well. The bottom panel of Fig. 4 shows the misalignment angle $\Delta \phi_{\mathrm{RA}}$ between the orientation angles $\phi_{\text {gal }}$ of the light distribution and $\phi_{\text {tot }}$ of the total matter distribution, as a function of the galaxy axis ratio $(b / a)_{e 1}^{\text {gal }}$ that was measured within $R_{\text {eff }}$. Larger scatter of $\Delta \phi_{\mathrm{RA}}$ for rounder galaxies is due to less clean measurements of their orientation angles. A marked evolution exists for the selected early-type galaxy samples at different redshifts, e.g., for a sub-sample of galaxies that have $(b / a)_{e 1}^{\text {gal }} \in[0.75,0.85]$, the standard deviation of $\Delta \phi_{\mathrm{RA}}$ measured within $R_{\text {eff }}$ decreases from $\sim 12^{\circ}$ at $z=1.0$ to $\sim 5^{\circ}$ at $z=0.6$, and finally to $\lesssim 2^{\circ}$ at $z \leqslant 0.3$. This evolution coincides with a mild increase of the (projected) central baryonic fraction towards lower redshifts (see Fig.(19). In the intervening period, stars and dark matter become more mixed and thus better aligned with cosmic time. These results are consistent with strong lensing observations (e.g., Sluse et al. 2012; Dye et al. 2014, Rusu et al. 2016). In particular, for galaxies that have stellar axis ratios falling within similar ranges as above, Gavazzi et al. (2012) measured an rms scatter of $18^{\circ}$ for the SL2S sample $(0.3<z<1.0)$ and Koopmans et al. (2006) reported an rms deviation of $3^{\circ}$ for the SLACS galaxy sample $(z<0.3)$.
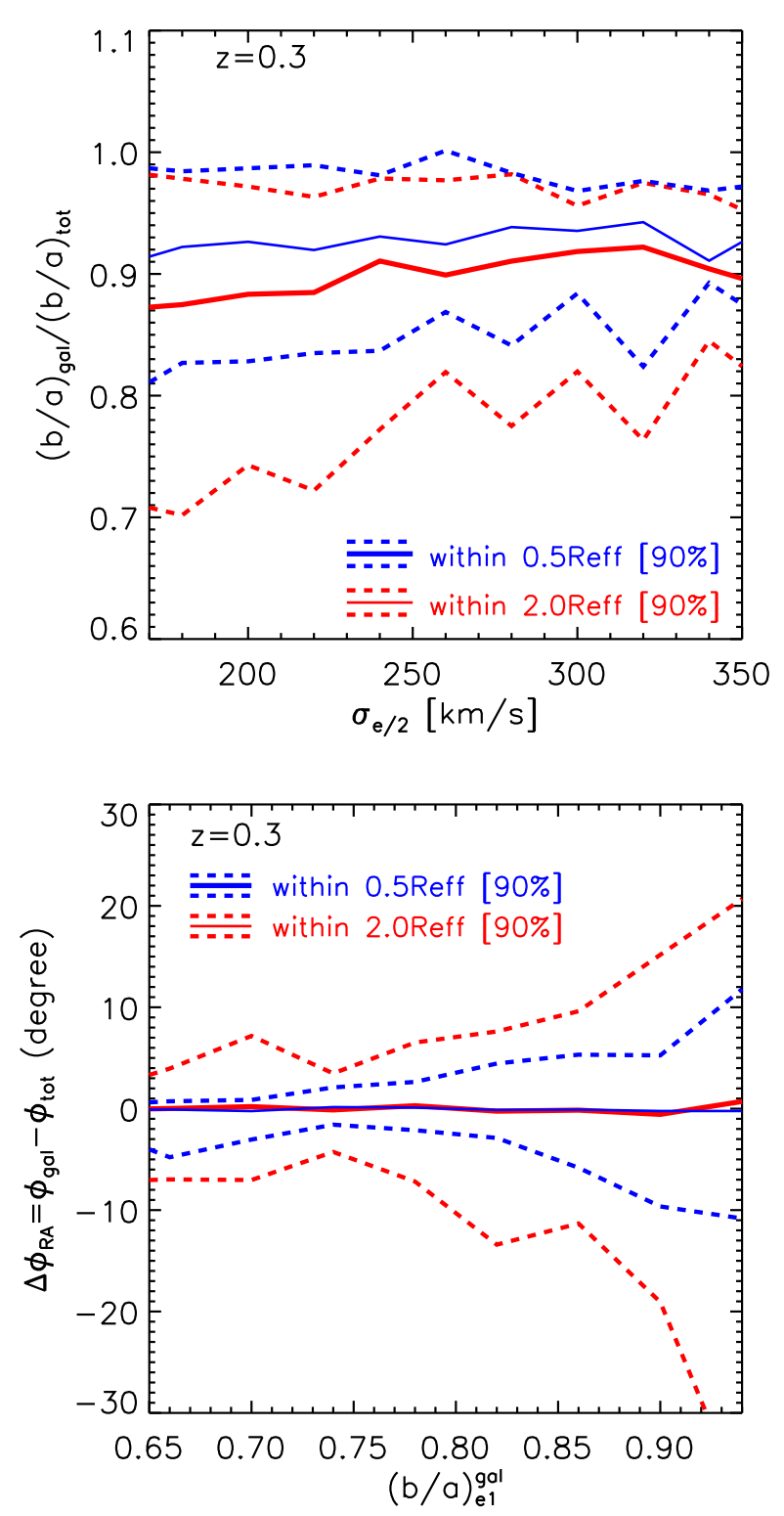

Figure 4. Top panel: the ratio between the axis ratio $b / a$ measured for the light distribution and that for the total matter distribution, as a function of the central stellar velocity dispersion $\sigma_{e / 2}$, for the selected early-type galaxy sample at $z=0.3$. Bottom panel: the difference between the orientation angle of the light distribution and that of the total matter distribution, as a function of the galaxy axis ratio $b / a_{e 1}^{\mathrm{gal}}$ measured within $R_{\mathrm{eff}}$, for the same galaxy sample. In both panels, blue and red represent the distributions measured within $0.5 R_{\text {eff }}$ and $2.0 R_{\text {eff }}$, respectively. The solid and the dashed lines indicate the median and the $90 \%$ boundaries of the distributions, respectively.

\subsection{Galaxy mass, size, and velocity dispersion}

The relations between galaxy size, velocity dispersion and stellar mass are among the most basic scaling relations. Fig. 5 shows the $\sigma_{e / 2}-M_{*}$ and $R_{\text {eff }}-M_{*}$ relations measured for the selected early-type galaxies at $z=0.1-0.4$. In either panel, the black squares mark the simulation data, and the solid and the dashed red lines indicate the best linear 
fit to the data and the $90 \%$ boundaries of the distribution, respectively. Note that the lower cut in the stellar velocity distribution (in the upper panel) is due to our sample selection criteria.

For comparison, the solid blue and cyans lines represent the best linearly fitted relations for the SLACS galaxies (Auger et al. 2010b), assuming either Salpeter IMFs (Salpeter 1955) or Chabrier IMFs (Chabrier 2003), respectively. Recent studies suggest that a Salpeter IMF is more compatible with observational inferences for massive earlytype galaxies (e.g., Auger et al. 2010a; Treu et al. 2010; Grillo \& Gobat 2010; Barnabè et al. 2011; Spiniello et al. 2011; Oguri et al. 2014; Sonnenfeld et al. 2015), while a Chabrier IMF is more suitable for those at the lower end of the spectrum (e.g., Shu et al. 2015). In addition, the linearly fitted relations for the observed early-type galaxies from the SDSS survey (Hvde \& Bernardi 2009a) are also given, as indicated by the solid orange lines.

As can be seen, there exists some slight underestimation of the central velocity dispersions for galaxies at the lower stellar-mass end. However, we note that the level of the disagreement between the simulation and the observations is comparable to that the systematic divisions among the observational relations themselves, which can be attributed to the uncertainty in the IMF and/or different sample biasing etc. Overall, the simulation broadly reproduces the observed $\sigma_{e / 2}-M_{*}$ and $R_{\mathrm{eff}}-M_{*}$ relations.

\subsection{The fundamental plane and the mass planes}

Elliptical galaxies are observed to tightly follow the socalled Fundamental Plane (hereafter FP; Faber et al. 1987; Diorgovski \& Davis 1987; Dressler et al. 1987) and its mass counterparts - the Stellar Mass Plane (hereafter $\mathrm{M}_{*} \mathrm{P}$; Hvde \& Bernardi 2009b) and Mass Plane (hereafter MP; Bolton et al. 2007). We examined how well the simulated galaxies trace these observed planes. To this end we adopted the same definitions as Auger et al. (2010b) and fitted the plane relations with the following form:

$$
\begin{aligned}
& \log \frac{R_{\mathrm{eff}}}{\mathrm{kpc}}=a+b \log \frac{\sigma_{e / 2}}{100 \mathrm{~km} \mathrm{~s}^{-1}}+c \log \Lambda, \\
& \Lambda=\frac{1}{2 \pi} \frac{L_{V}}{10^{9} L_{\odot}}\left(\frac{R_{\mathrm{eff}}}{\mathrm{kpc}}\right)^{-2}(\text { for } \mathrm{FP}), \\
& \Lambda=\frac{1}{2 \pi} \frac{M_{*}}{10^{9} M_{\odot}}\left(\frac{R_{\mathrm{eff}}}{\mathrm{kpc}}\right)^{-2}\left(\text { for } \mathrm{M}_{*} \mathrm{P}\right), \\
& \Lambda=\frac{4}{\pi} \frac{M_{\mathrm{tot}}\left(\leqslant 0.5 R_{\mathrm{eff}}\right)}{10^{10} M_{\odot}}\left(\frac{R_{\mathrm{eff}}}{\mathrm{kpc}}\right)^{-2}(\text { for } \mathrm{MP}) .
\end{aligned}
$$

In Eq. (11), $L_{V}$ is the Sersic luminosity measured in the rest-frame Johnson- $V$ band. A measurement uncertainty of $5 \%$ was assumed for each of the five quantities of the simulated galaxies. The fitting of the coefficients $a, b$ and $c$ was done using of the LTS_PLANEFIT program described in Cappellari et al. (2013), which combines the Least Trimmed Squares robust technique of Rousseeuw \& Van Driessen (2006) with a least-squares fitting algorithm which allows for errors in all variables as well as intrinsic scatter.

Fig. 6] shows the FP relation of the selected early-type galaxy sample at $z=0.3$ from the simulation. The blue circles filled with black dots indicate individual galaxies. The
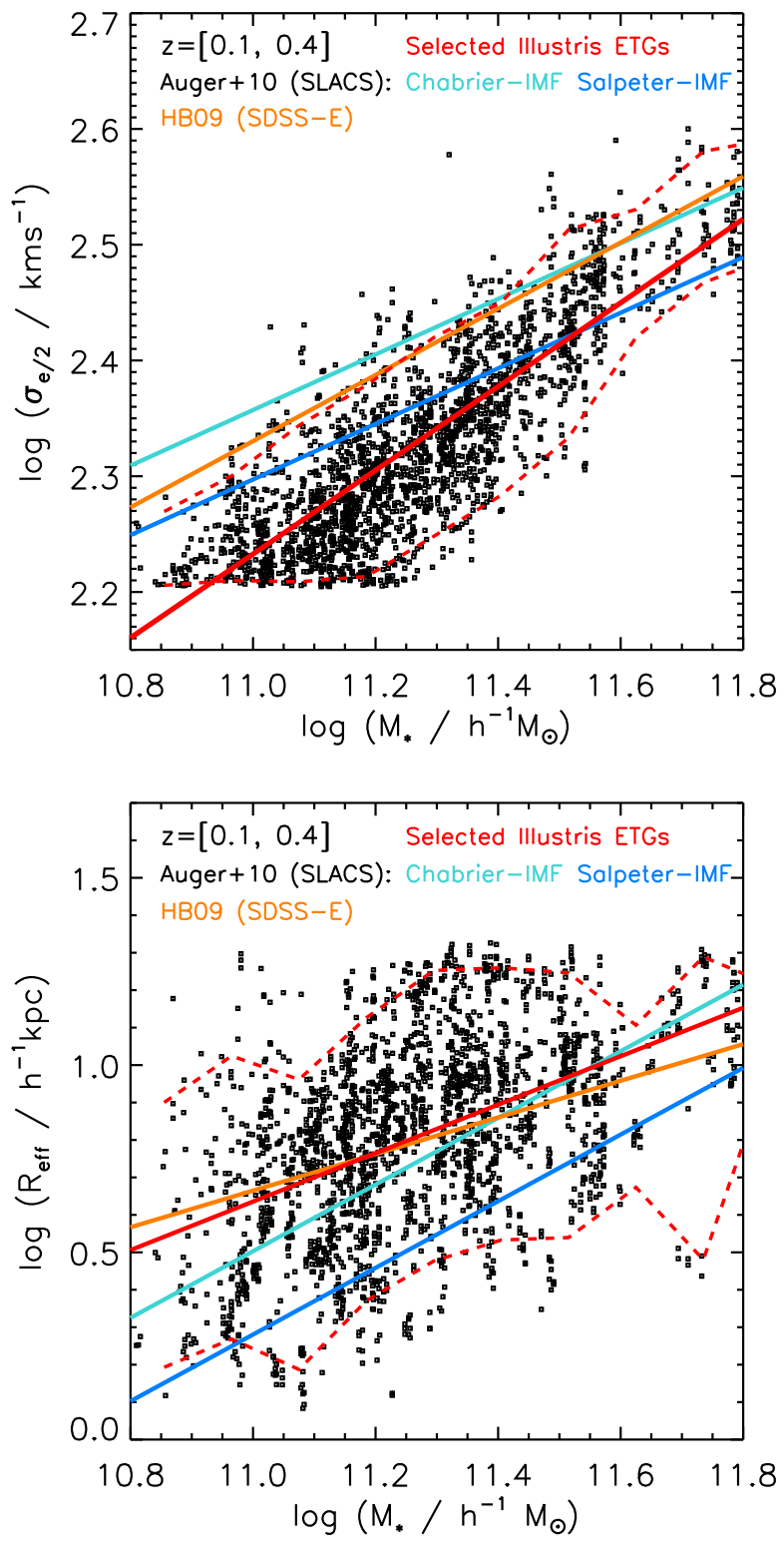

Figure 5. The $\sigma_{e / 2}-M_{*}$ (top panel) and $R_{\text {eff }}-M_{*}$ (bottom panel) relations. The black squares mark the simulation data of the selected early-type galaxies at $z=0.1-0.4$. The lower cut in stellar velocity distribution (in the top panel) is due to our selection criteria. The solid and the dashed red lines indicate the best linear fit to the data and the $90 \%$ boundaries of the distribution, respectively. For comparison, the solid blue and cyans lines represent the best linearly fitted relations for the SLACS galaxies (Auger et al. 2010b), assuming either Salpeter IMFs or Chabrier IMFs, respectively. The solid orange lines indicate the linearly fitted relations for the observed early-type galaxies from the SDSS survey (Hyde \& Bernardi 2009a).

red solid and dashed lines show $2.6 \sigma$ and $1 \sigma$ scatter, respectively, around the best-fitting relation, which is given by the black solid line. Galaxies indicated by green symbols are outside $3 \sigma$ of the fitted relation. For the $\mathrm{FP}$ relation, we found best-fitting coefficients equal to $a=0.10 \pm 0.02$, $b=1.42 \pm 0.05$ and $c=-0.65 \pm 0.01$. For the $\mathrm{M}_{*} \mathrm{P}$, we obtained $a=0.43 \pm 0.01, b=1.11 \pm 0.04$ and $c=-0.54 \pm 0.01$; 


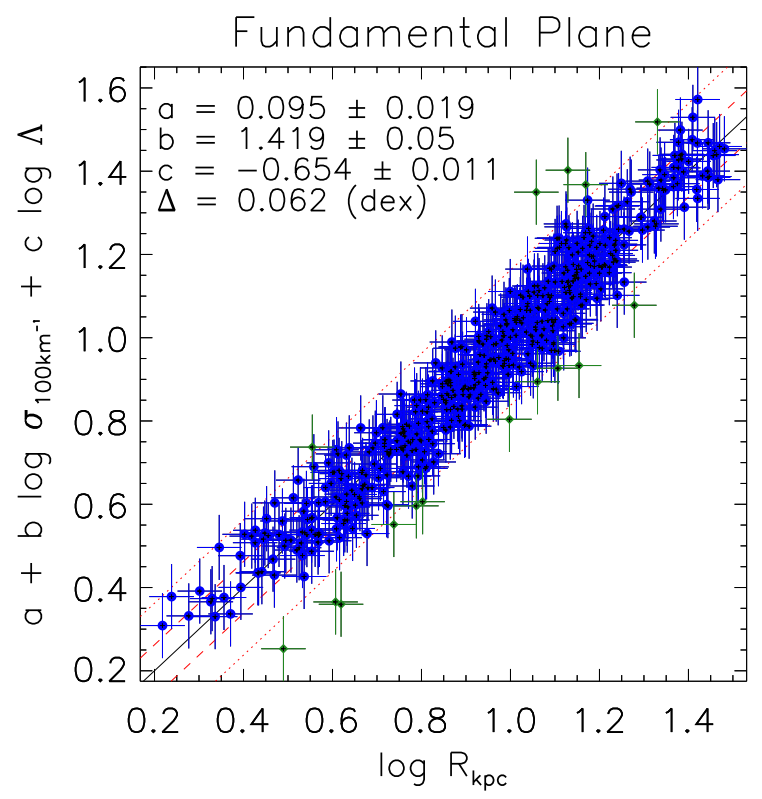

Figure 6. The fundamental plane relation of the selected earlytype galaxy sample at $z=0.3$ from the simulation. $R_{\mathrm{kpc}}$ is $R_{\text {eff }} / \mathrm{kpc}$ and $\sigma_{100 \mathrm{~km} \mathrm{~s}^{-1}}$ is $\sigma_{e / 2} /\left(100 \mathrm{~km} \mathrm{~s}^{-1}\right)$. A measurement uncertainty of $5 \%$ was assumed for each "observed" quantity. The blue circles filled with black dots indicate individual simulated galaxies. The red solid and dashed lines give the $2.6 \sigma$ and $1 \sigma$ bounds, respectively, from the best-fitting relation given by the black solid line. Galaxies indicated by green symbols are outside $3 \sigma$ of the fitted relation. The fitting and plotting software LTS_PLANEFIT used here was provided by Cappellari et al. (2013).

and for MP, $a=-0.04 \pm 0.02, b=1.63 \pm 0.06$ and $c=-0.96 \pm 0.02$.

The fitting coefficient $a$ represents the normalizations of the plane relations; while $b$ and $c$ depict the slopes of the plane relations. In particular, coefficients $b$ and $c$ depend on the sample selection, observational bands, and fitting methods (see Bernardi et al. 2003). Observationally, $b$ ranges from 0.99 to 1.52 , and $c$ ranges from -0.88 to -0.74 for the $\mathrm{FP}$; for the MP, $b$ varies from 1.77 to 1.86 , and $c$ ranges from -1.30 to -0.83 (Bernardi et al. 2003; Bolton et al.; Bolton et al. 2008b; Auger et al. 2010b; Cappellari et al. 2013). Within the fitting uncertainties, our bestfitting values for $a, b$ and $c$ are not in stark disagreement with observational results. A more detailed study of the FP relation of the Illustris early-type galaxies will be presented in a separate paper ( $\mathrm{Li}$ et al. in preparation).

\section{STELLAR ORBITAL ANISOTROPIES}

One of the major uncertainties in interpreting kinematical data is the stellar orbital anisotropy, which cannot be directly measured or constrained with high accuracy (e.g., Li et al. 2016) as it is degenerate with galaxy density slopes, unless the latter can be determined independently. When combined with single-aperture stellar kinematics, strong lensing studies often have to assume zero orbital anisotropies (but see e.g., Barnabè et al. 2009, 2011 for 2-D kinematics studies of the SLACS lenses). In this section, we thus investigate the dependencies of the anisotropy parameter $\beta$ and its redshift evolution since $z=1.0$ to check the validity of this assumption.

For spherically symmetric systems, the anisotropy parameter $\beta$ can be written as (Binney \& Tremaine 2008)

$$
\beta=1-\frac{\overline{V_{\phi}^{2}}+\overline{V_{\theta}^{2}}}{2 \overline{V_{r}^{2}}}=1-\frac{\sigma_{\phi}^{2}+\sigma_{\theta}^{2}}{2 \sigma_{r}^{2}},
$$

where $\overline{V_{\phi}^{2}}, \overline{V_{\theta}^{2}}$ and $\overline{V_{r}^{2}}$ are the second velocity moments, and $\sigma_{\phi}^{2}, \sigma_{\theta}^{2}$ and $\sigma_{r}^{2}$ are the velocity dispersions measured in the azimuthal $\hat{\phi}$, polar $\hat{\theta}$ and radial $\hat{r}$ directions of a spherical coordinate system. By definition, $\sigma^{2} \equiv \overline{V^{2}}-\bar{V}^{2}$, where $\bar{V}$ is the mean velocity. The second equality sign in Eq. (14) is valid for stationary non-rotating systems, where $\overline{V_{\phi}}, \overline{V_{\theta}}$ and $\overline{V_{r}}$ vanish. Note that observationally, as the measurements are carried out for the light components, $\overline{V_{\phi}}=0, \overline{V_{\theta}}=0$ and $\overline{V_{r}}=0$ by construction. We followed the same convention, measuring $\beta$ through $\sigma^{2}$ instead of $\overline{V^{2}}$. In this sense, $\beta$ is constructed to measure the anisotropy of the velocity dispersion. $\beta=0$ corresponds to the "isotropic" case, and $\beta>0(\beta<0)$ describes a radially (tangentially) anisotropic orbital distribution.

For each simulated galaxy, measurements of $\beta$ were made (for stellar particles) within 3 -D radii of $0.5 R_{\text {eff }}$ and $2.0 R_{\text {eff }}$ from the centres of galaxies. Fig. 7 shows the dependence of $\beta$ on $\sigma_{e / 2}$ for the selected galaxies at $z=0.3$. The blue and red curves present the distributions of $\beta(r \leqslant$ $\left.0.5 R_{\text {eff }}\right)$ and $\beta\left(r \leqslant 2.0 R_{\text {eff }}\right)$, respectively. The solid and dashed lines indicate the medians and the $90 \%$ percentiles, respectively. The stellar orbits of more massive galaxies tend to be more radially anisotropic than those of their lowermass counterparts. Observationally, Koopmans et al. (2009) applied two independent techniques to measure the logarithmic density slopes for SLACS early-type galaxie: 4 . The combination of the two measurements provided a (weak) constraint on the orbital anisotropies, $\langle\beta\rangle=0.45 \pm 0.25$, consistent with the values we measured for their counterparts in the Illustris simulation.

It is interesting to note that the different behaviour of the average $\beta$ in low- and high-mass galaxies is possibly related to the recent star formation histories. To demonstrate this, we consider in Fig. 8 the central (cold) gas fractions versus $\sigma_{e / 2}$ for the same galaxy sample at $z=0.3$. The median and $90 \%$ boundaries of the distribution are given by the solid and dashed lines, respectively. The blue and red curves show the distributions of the cold (HI) and total gas fractions, respectively, measured within a 3-D radius of $R_{\text {eff }}$ from the galaxy centres. We can see that more massive galaxies that tend to have higher radial anisotropies also contain less cold gas in their central regions, while the less massive galaxies with higher tangential velocity contributions have on average higher central gas fractions. The former were also seen to have Johnson $B-V$ colours redder than the latter. We also note that galaxies at higher redshifts are markedly bluer and contain higher fractions of central cold gas than their lower redshift counterparts.

These correlations provide a consistent picture. As the cold gas is channelled down to the centre, star-formation

4 The majority of the SLACS galaxies are located at lower redshifts $(z<0.3)$ and have $\sigma_{e / 2}$ peaks around $\sim 250 \pm 40 \mathrm{~km} \mathrm{~s}^{-1}$. 


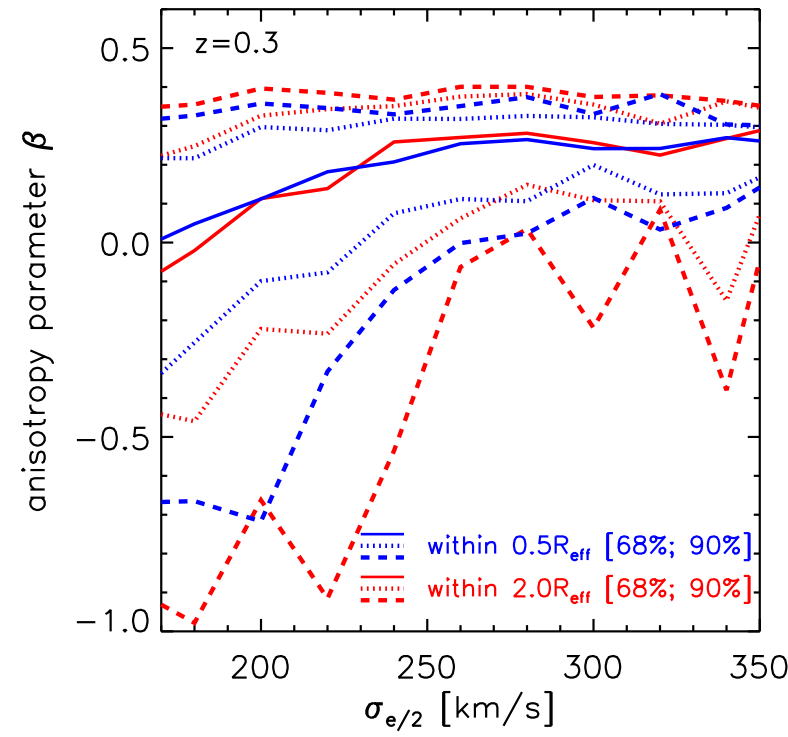

Figure 7. The anisotropy parameter $\beta$ as a function of the central stellar velocity dispersion $\sigma_{e / 2}$, measured for the selected earlytype galaxies at $z=0.3$. The blue and red curves present the distributions of $\beta\left(r \leqslant 0.5 R_{\text {eff }}\right)$ and $\beta\left(r \leqslant 2.0 R_{\text {eff }}\right)$, respectively. The solid, dotted and dashed lines indicate the medians, the $68 \%$ and $90 \%$ boundaries of the distributions, respectively.

activity preferably happens on tangential orbits as a consequence of gas accretion and rotational support. When the system (passively) evolves, more radial anisotropies emerge. In this case, one would expect that the stellar orbits at higher redshifts are more tangentially dominated than their lower-redshift counterparts.

Indeed, this can be clearly seen in Fig.9, which shows the redshift evolution of $\beta$ since $z=1$.0. In order to quantify this redshift evolution, we randomly assigned a galaxy from a given snapshot at $z_{0}$ with a redshift of $z=z_{0} \pm \Delta z / 2$, where $\Delta z \leqslant 0.05$. We then fit $\beta$ versus $z$ using a linear regression approach, which resulted in $\partial \beta\left(r \leqslant 0.5 R_{\text {eff }}\right) / \partial z=$ $-0.41 \pm 0.02$ with a linear correlation coefficient $r=-0.33$ and $\partial \beta\left(r \leqslant 2.0 R_{\text {eff }}\right) / \partial z=-0.37 \pm 0.02$ with $r=-0.26$. We verify that changing the range of $\Delta z$ from 0.01 to 0.1 makes no difference in the linear regression results.

We mention in passing that the observed correlation between $\beta$ and $\sigma_{e / 2}$ could strongly depend on the details of the adopted galactic wind and AGN feedback models, which, as shown in Genel et al. (2015), efficiently affect the gas distribution and determine the stellar angular momentum and thus orbital anisotropies. Observational constraints on the distribution of $\beta$ are crucial in establishing the validity of various feedback models.

\section{PROJECTED CENTRAL DARK MATTER FRACTIONS}

The projected central dark matter fraction $f_{\mathrm{dm}}$ of observed galaxies has often been constrained through combined measurements of the stellar and the total masses. A galaxy's stellar mass can be obtained using the SPS method applied to

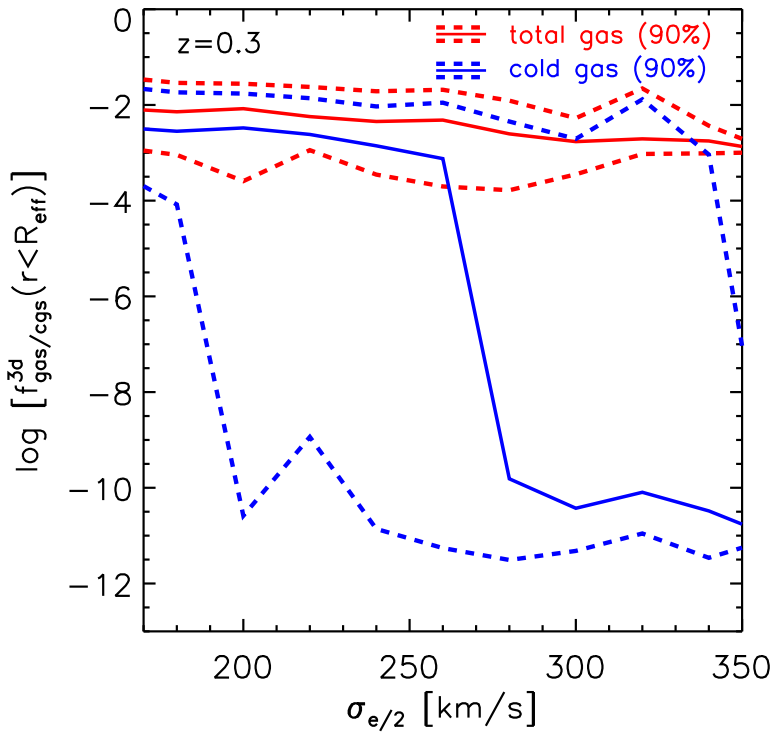

Figure 8. The central (cold) gas fractions versus $\sigma_{e / 2}$ for the early-type galaxy sample at $z=0.3$. The median and $90 \%$ boundaries of the distribution are given by the solid and the dashed lines, respectively. The blue and red curves show the distributions of the cold (HI) and of the total gas fractions within a 3-D radius of $R_{\text {eff }}$, respectively.

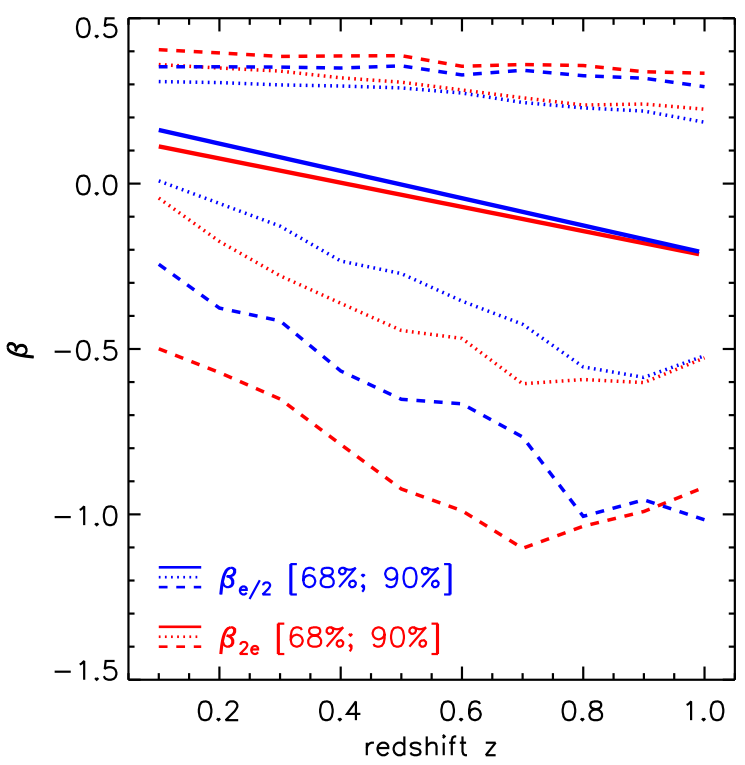

Figure 9. The redshift evolution of the anisotropy parameter $\beta$ measured for the early-type galaxy samples at $z=0.1-1.0$ from the simulation. The blue and red curves represent the distributions of $\beta\left(\leqslant 0.5 R_{\text {eff }}\right)$ and $\beta\left(\leqslant 2.0 R_{\text {eff }}\right)$, respectively. The solid, dotted and dashed lines indicate the best linear fit to the data, the $68 \%$ and $90 \%$ boundaries of either distribution, respectively. Linear regression resulted in $\partial \beta\left(r \leqslant 0.5 R_{\text {eff }}\right) / \partial z=$ $-0.41 \pm 0.02$ with a linear correlation coefficient $r=-0.33$ and $\partial \beta\left(r \leqslant 2.0 R_{\text {eff }}\right) / \partial z=-0.37 \pm 0.02$ with $r=-0.26$. 
multi-band photometric data, provided that the IMF is independently constrained. Constraints on the total mass may come from stellar kinematics and/or strong lensing measurements. As already discussed in Sect.4, this is often complicated due to the lack of knowledge about stellar orbital anisotropies. In addition, it also needs to assume parameterized density profiles either of the total matter distribution (e.g., Koopmans et al. 2006; Auger et al. 2010b) or of the individual dark and luminous components (e.g., Cappellari et al. 2013; Sonnenfeld et al. 2015; Li et al. 2016).

In this section, we first compare the projected central dark matter fraction of the early-type Illustris galaxies to the ones derived for the SLACS (Auger et al. 2010b) and SL2S (Sonnenfeld et al. 2015) galaxies. To this end, we use the quantity $f_{\mathrm{dm}}(R \leqslant 5 \mathrm{kpc})$, which is defined as the projected dark matter fraction within a fixed aperture of $5 \mathrm{kpc}$. The choice of a fixed aperture for comparison purposes instead of, say, $R_{\text {eff }}$, is to eliminate systematic differences due to possible sample bias.

Fig. 10] shows $f_{\mathrm{dm}}(R \leqslant 5 \mathrm{kpc})$ as a function of the central velocity dispersion $\sigma_{e / 2}$. The blue curves give the distribution of the directly measured $f_{\mathrm{dm}}(R \leqslant 5 \mathrm{kpc})$ for the selected early-type galaxies at $z=0.1-1.0$, whereas the red curves indicate the distribution of measurements for the same galaxies but obtained by modelling the total matter density distributions with power-law profiles (see Sect.6.1.3 for details). The solid and the dashed lines give the median and the $90 \%$ boundaries of the simulation distributions. The black squares indicate measurements for the SLACS and SL2S galaxy samples, where the error bars show $1 \sigma$ error of the data. Specifically, a dark halo component was modelled by a NFW profile, and a de-projected best-fitting de Vaucouleur distribution was adopted to model the stellar distribution. The sum of the two was then used to fit both strong lensing and kinematics data (Sonnenfeld et al. 2015).

As can be seen from the figure, the distribution of the measurements derived under the power-law profile assumption (in red) has larger scatter than the true distribution (in blue). But both distributions indicate that dark matter on average contributes with $40 \%-50 \%$ to the (projected) total matter distributions in centres of early-type Illustris galaxies. This fraction is higher than suggested by the observational results. Such tension poses a potential challenge to the stellar formation and feedback models adopted by the simulation.

Observations also suggest that the projected central dark matter fraction within the effective radius $f_{\mathrm{dm}}(R \leqslant$ $\left.R_{\text {eff }}\right)$ is mass-dependent: the more massive galaxies are, the larger is their dark matter fraction. A noticeable positive correlation was also found between $f_{\mathrm{dm}}\left(R \leqslant R_{\text {eff }}\right)$ and $R_{\text {eff }}$, albeit with large scatter (e.g., Tortora et al. 2009; Napolitano et al. 2010; Humphrev \& Buote 2010; Graves \& Faber 2010; Auger et al. 2010b; Shu et al. 2015). Using a significantly larger statistical sample of early-type galaxies from the simulation, we investigated such dependences.

Fig.11 presents $f_{\mathrm{dm}}(\leqslant R)$ versus $\sigma_{e / 2}$ (top panel) and versus $R_{\text {eff }}$ (bottom panel) for the early-type galaxy sample selected at $z=0.3$. Blue and red represent the fractions measured within a radius of $0.5 R_{\text {eff }}$ and $R_{\text {eff }}$, respectively. The solid and the dashed lines give the best linear fit to the data and the $90 \%$ boundaries of the simulation distribution.
Linear regression resulted in $\partial f_{\mathrm{dm}}\left(R \leqslant 0.5 R_{\mathrm{eff}}\right) / \partial \sigma_{e / 2}=$ $0.0001 \pm 0.0001$ with a linear correlation coefficient $r=0.05$; $\partial f_{\mathrm{dm}}\left(R \leqslant R_{\text {eff }}\right) / \partial \sigma_{e / 2}=0.0002 \pm 0.0001$ with $r=0.09$ and $\partial f_{\mathrm{dm}}\left(R \leqslant 0.5 R_{\text {eff }}\right) / \partial \lg R_{\text {eff }}=0.40 \pm 0.01$ with $r=0.89$; $\partial f_{\mathrm{dm}}\left(R \leqslant R_{\text {eff }}\right) / \partial \lg R_{\text {eff }}=0.41 \pm 0.01$ with $r=0.90$.

As can be seen, for the early-type galaxy sample, the dependence on the stellar velocity dispersions is much weaker than on galaxy sizes. The latter shows a tight and clear positive correlation between the two quantities. Similar dependences were also found by Remus et al. (2016), where earlytype galaxies selected from the Magneticum Pathfinder Simulations (Dolag et al. 2015) were studied.

These dependences suggest that the projected central dark matter fraction in terms of $f_{\mathrm{dm}}\left(R \leqslant R_{\mathrm{eff}}\right)$ [or $f_{\mathrm{dm}}(R \leqslant$ $\left.0.5 R_{\text {eff }}\right)$ ] has a very mild mass dependence for our earlytype galaxy samples. The clear positive correlation between $f_{\mathrm{dm}}\left(R \leqslant R_{\text {eff }}\right)$ [or $f_{\mathrm{dm}}\left(R \leqslant 0.5 R_{\text {eff }}\right)$ ] and $\lg R_{\text {eff }}$ may purely be an aperture effect: the dark matter fraction drops with decreasing radius as baryons dominate more and more towards the galactic centre (also see Grillo 2010; and Fig. 3 of Xu et al. 2016).

We also studied the redshift dependence of the projected central dark matter fraction $f_{\mathrm{dm}}(\leqslant R)$ for the selected early-type galaxy samples in different redshift bins between $z=0.1$ to $z=1.0$. The result is shown in Fig. 12 , where the solid, dotted and dashed lines indicate the best linear fit to the data, the $68 \%$ and $90 \%$ boundaries of the distribution, respectively. Using linear regression approach, we found that both $\partial f_{\mathrm{dm}}\left(R \leqslant 0.5 R_{\text {eff }}\right) / \partial z$ and $\partial f_{\mathrm{dm}}\left(R \leqslant R_{\text {eff }}\right) / \partial z$ are equal to $0.04 \pm 0.01$ and both fits have linear correlation coefficients $r=0.09$.

Fair comparisons with observations (or studies using simulations of the same kind) would require applying identical sample selection criteria. Dye et al. (2014) reported a similar increasing trend of $f_{\mathrm{dm}}\left(R \leqslant 0.5 R_{\text {eff }}\right)$ with redshift for a galaxy sample from the Herschel Astrophysical Terahertz Large Area Survey (see also Sonnenfeld et al. 2015, Fig. 6). The result from the simulation is not in stark contrast with observations. We note that the early-type galaxy samples at different redshifts were selected according to their $\sigma_{e / 2}$, which also evolve with redshift for individual galaxies. Therefore, the redshift trends found here hold for a statistical sample defined as such, but not necessarily for individual galaxies (also see Remus et al. 2016).

\section{CENTRAL MATTER DENSITY PROFILES}

As already shown in Sect. 5, baryons contribute a large fraction of the total matter distribution in centres of early-type galaxies. Fig.13 shows the density distribution of an earlytype galaxy at $z=0.3$ (the same galaxy as presented in Fig. 3). The black, red and blue symbols indicate the density distributions of the total, dark matter and baryonic matter, respectively. The profile of baryons is much steeper than that of dark matter in the central region. Interestingly, the projected galactocentric radii where typical strong lensing and stellar kinematics data are available coincide with the radii where the radial profiles of dark matter and baryons intercept (e.g., see Fig. 3 of Xu et al. 2016). In this radial range (normally $0.5-2.0 R_{\mathrm{E}}$, corresponding to the inner few $\mathrm{kpc}$ ), the slope of the total density profile depends on both 


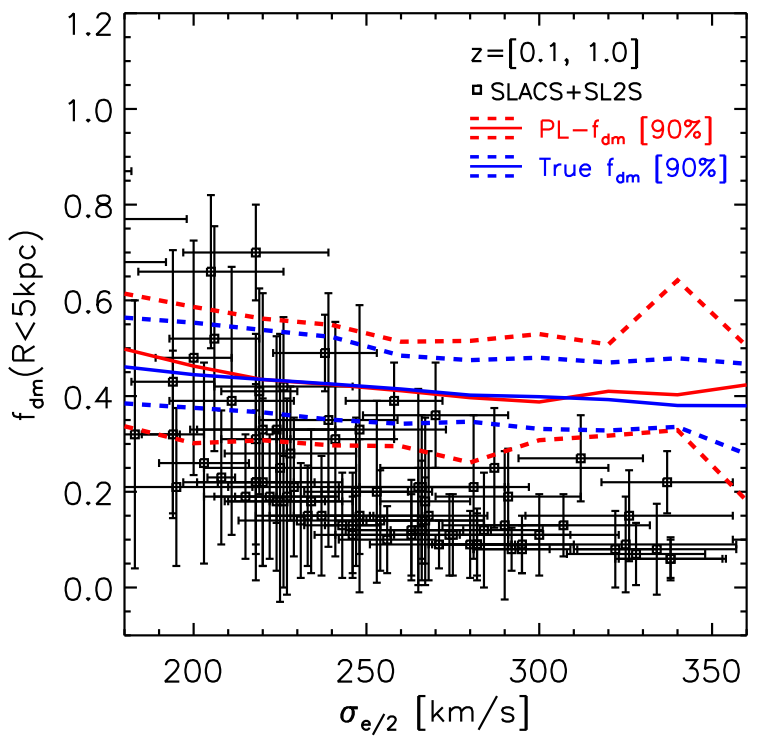

Figure 10. The projected central dark matter fraction $f_{\mathrm{dm}}(R \leqslant$ $5 \mathrm{kpc})$ as a function of the central velocity dispersion $\sigma_{e / 2}$. The blue curves present the distribution of the directly measured $f_{\mathrm{dm}}(R \leqslant 5 \mathrm{kpc})$ for the selected early-type galaxies at $z=0.1-1.0$. The red curves indicate the distribution of the measurements for the same galaxies but obtained by assuming the total matter density distributions of galaxies to be power laws. The solid and the dashed lines give the median and the $90 \%$ boundaries of the simulation distributions. The black squares show measurements for the SLACS and SL2S galaxy samples, where the error bars indicate $1 \sigma$ error of the data. For the observed galaxy sample, a dark halo component was modelled by a NFW profile, and a de-projected best-fitting de Vaucouleur distribution was adopted to model the stellar distribution. The sum of the two was then used to fit both strong lensing and kinematics data (see Sonnenfeld et al. 2015 for details).

components. Its quantification can be problematic because the sum of the two components does not necessarily obey simple global power-law distributions, i.e., no single slope can describe the overall distribution.

For a simulated galaxy, one can find approximate slope estimators within given radial ranges. For example, an average slope $\gamma^{\mathrm{AV}}\left(r_{1}, r_{2}\right)$ of the density profile between two radii $r_{1}$ and $r_{2}$ can be expressed using a power-law interpolation (the superscript "AV" refers to "average"):

$$
\gamma^{\mathrm{AV}}\left(r_{1}, r_{2}\right) \equiv \frac{\ln \left[\rho\left(r_{2}\right) / \rho\left(r_{1}\right)\right]}{\ln \left(r_{1} / r_{2}\right)}
$$

One can also define $\gamma^{\mathrm{PL}}\left(r_{1}, r_{2}\right)$ as the local logarithmic slope of the power-law profile that best fits the radial density distribution between $r_{1}$ and $r_{2}$ (the superscript "PL" refers to "power-law"). In particular, this definition has been adopted in many studies on simulated galaxies (e.g., Nipoti et al. 2009a; Johansson et al. 2012; Remus et al. 2013; Li et al. 2016) when comparing to observations.

Another definition is a mass-weighted density slope $\gamma^{\mathrm{MW}}(r)$ (the superscript "MW" refers to "mass-weighted",
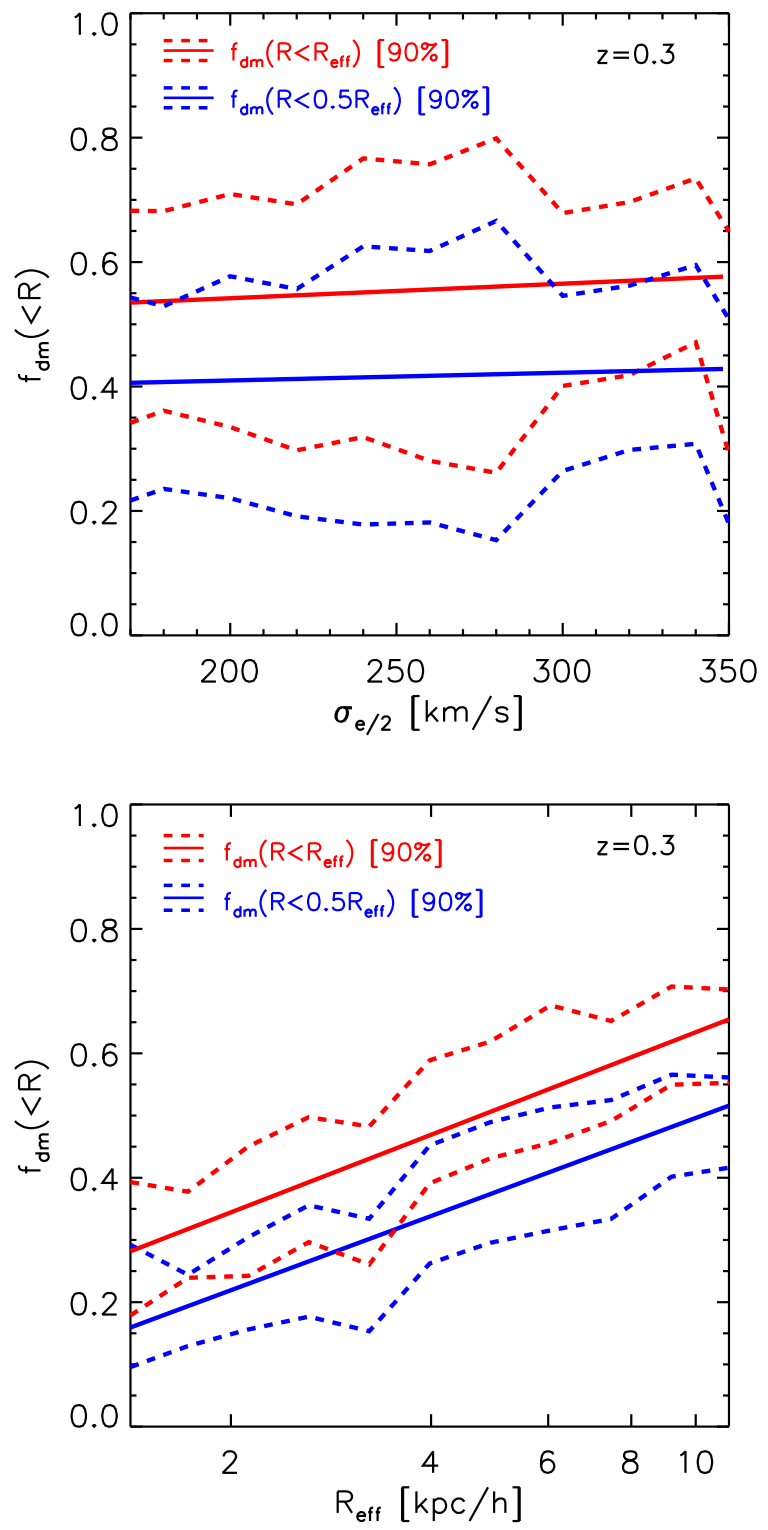

Figure 11. The projected central dark matter fraction $f_{\mathrm{dm}}(\leqslant R)$ as a function of the central velocity dispersion $\sigma_{e / 2}$ (top panel) and of the effective radius $R_{\text {eff }}$ (bottom panel) for the selected early-type galaxy sample at $z=0.3$. Blue and red represent the fractions measured within a projected radius of $0.5 R_{\text {eff }}$ and $R_{\text {eff }}$, respectively. The solid and the dashed lines give the best linear fit to the data and the $90 \%$ boundaries of the simulation distribution, respectively. Linear regression resulted in $\partial f_{\mathrm{dm}}(R \leqslant$ $\left.0.5 R_{\text {eff }}\right) / \partial \sigma_{e / 2}=0.0001 \pm 0.0001$ with a linear correlation coefficient $r=0.05 ; \partial f_{\mathrm{dm}}\left(R \leqslant R_{\text {eff }}\right) / \partial \sigma_{e / 2}=0.0002 \pm 0.0001$ with $r=0.09$ and $\partial f_{\mathrm{dm}}\left(R \leqslant 0.5 R_{\text {eff }}\right) / \partial \lg R_{\text {eff }}=0.40 \pm 0.01$ with $r=0.89 ; \partial f_{\mathrm{dm}}\left(R \leqslant R_{\mathrm{eff}}\right) / \partial \lg R_{\mathrm{eff}}=0.41 \pm 0.01$ with $r=0.90$.

see Dutton \& Treu 2014):

$$
\begin{aligned}
\gamma^{\mathrm{MW}}(r) & \equiv \frac{1}{M(r)} \int_{0}^{r} 4 \pi x^{2} \rho(x) \gamma(x) \mathrm{d} x \\
& =3-\frac{4 \pi r^{3} \rho(r)}{M(r)}=3-\frac{\mathrm{d} \ln M(r)}{\mathrm{d} \ln r}
\end{aligned}
$$




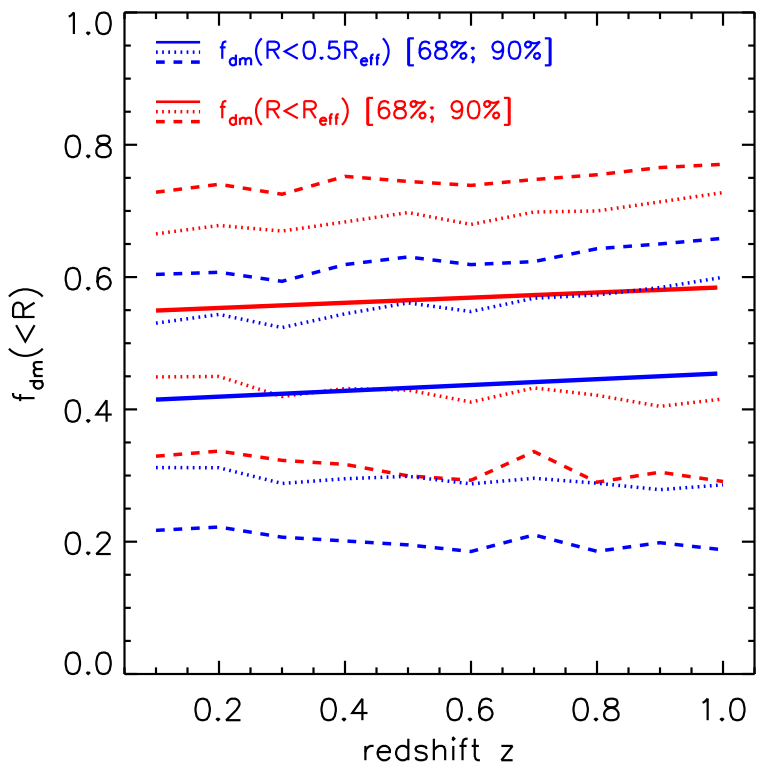

Figure 12. The redshift evolution of the central dark matter fractions $f_{\mathrm{dm}}\left(R \leqslant 0.5 R_{\text {eff }}\right)$ (blue) and $f_{\mathrm{dm}}\left(R \leqslant R_{\text {eff }}\right)$ (red) measured for the selected early-type galaxy samples at $z=0.1-1.0$. The solid, dotted and dashed lines indicate the best linear fit to the data, the $68 \%$ and $90 \%$ boundaries of either distribution, respectively. For the entire sample, both $\partial f_{\mathrm{dm}}\left(R \leqslant 0.5 R_{\mathrm{eff}}\right) / \partial z$ and $\partial f_{\mathrm{dm}}\left(R \leqslant R_{\text {eff }}\right) / \partial z$ are equal to $0.04 \pm 0.01$ and both fits have linear correlation coefficient $r=0.09$.

where the local slope $\gamma(r)$ is given by

$$
\gamma(r) \equiv-\frac{\mathrm{d} \ln \rho(r)}{\mathrm{d} \ln r},
$$

and the 3-D enclosed total mass $M(r)$ is given by

$$
M(r) \equiv \int_{0}^{r} 4 \pi x^{2} \rho(x) \mathrm{d} x .
$$

Note that for a matter density distribution that follows a perfect power law, i.e., $\rho(r) \propto r^{-\gamma^{\mathrm{PL}}}, \gamma^{\mathrm{MW}}(r)=\gamma^{\mathrm{PL}}$ at all radii $r$.

Despite different definitions, all the above-mentioned slope estimators quantify some intrinsic matter density distribution to first-order. The interpretation of the resulting measurements under these definitions is straightforward and model-independent. However, they cannot be applied to observed galaxies, unlike the brightness distribution, which can be directly measured as long as the galaxy is spatially resolved. The (total) matter density distribution can only be determined indirectly by dynamical methods, for example gravitational lensing or stellar kinematics, and through fitting parameterized models based on certain assumptions.

For galaxies at lower redshifts where 2-D kinematical data (e.g., the integral-field spectroscopic data) are available, one can implement sophisticated dynamical methods (e.g., Barnabè et al. 2011; Cappellari et al. 2015). Assuming parameterized density profiles within radial ranges (e.g., from $\sim 0.1 R_{\text {eff }}$ to a few $\left.R_{\text {eff }}\right)$ for data fitting purposes, the method allows simultaneous fitting to the matter distribution as well as the stellar orbital anisotropies. In particular, Li et al. (2016) investigated the validity of such techniques using the Illustris simulation. They found that although the

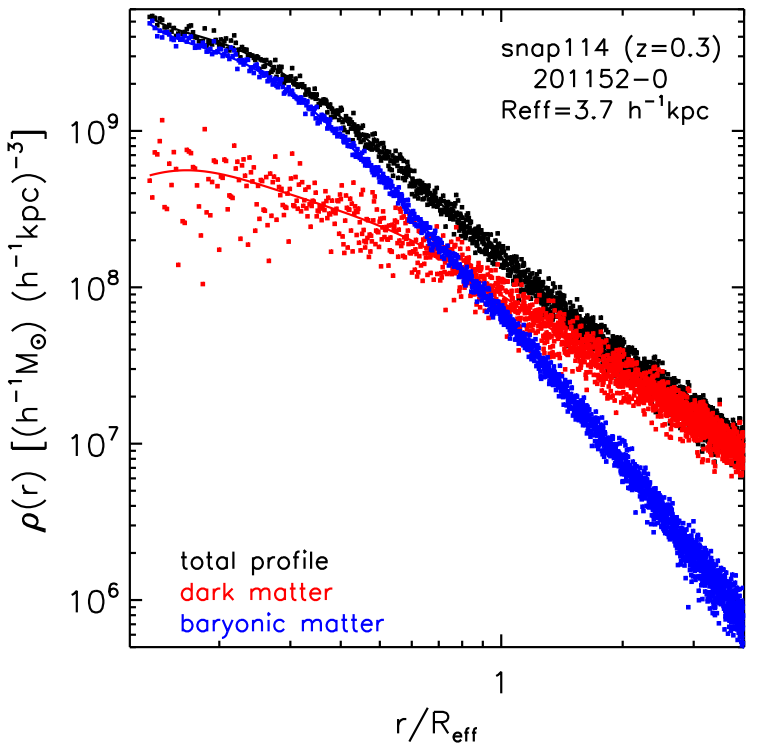

Figure 13. Matter density distribution of an early-type galaxy at $z=0.3$ (the same galaxy as presented in Fig. 3). The black, red and blue points indicate the density distributions of the total, dark matter and baryonic matter, respectively.

orbital anisotropies cannot be accurately recovered and degeneracies exist between the dark matter and stellar components, the total mass distributions and their density slopes

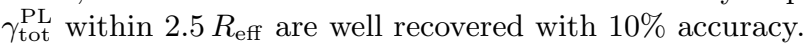

For galaxies at higher redshifts where only singleaperture kinematical data are available, simple approaches that use multiple mass measurements at different radii to make predictions about matter density slopes can be adopted. For example, in the SLACS (Auger et al. 2010b) and SL2S (Sonnenfeld et al. 2013, 2015) surveys, the central density slopes were derived for the observed lensing galaxies assuming spherical symmetry, power-law profiles, and isotropic orbital distributions $(\beta=0)$. The derived slopes could therefore suffer from more systematic biases than those using 2-D kinematics methods.

In order to make fair comparisons, one should apply the observational estimators also to the simulated samples. In this work, we adopted a simple approach along these lines that combines strong lensing and single-aperture kinematics for simulated early-type galaxies. In Sect. 6.1, we present the derived slopes, compare them with observational results, and discuss two associated major systematic effects. In Sect. 6.2, different slope estimators (as presented above) of the total matter density distributions are compared. In Sect. 6.3, we present the inner density slopes of the dark matter and stellar distributions of the early-type Illustris galaxies, and in Sect. 6.4, their cosmic evolution is discussed.

\subsection{Total density slopes from combining strong lensing and single-aperture kinematics}

For making fair comparisons of the total power-law slopes between the simulation and, in particular, the SLACS and SL2S survey results, we adopted a similar practice as used in these studies. Here we first briefly describe the main features 
of the method (details can be found in e.g., Koopmans et al. 2006; Auger et al. 2010b; Sonnenfeld et al. 2013).

The total matter distribution of a galaxy is assumed to be spherically symmetric, with a radial profile that follows a power law, i.e., $\rho(r) \propto r^{-\gamma^{\prime}}$. The stellar distribution is obtained by de-projecting the Sersic profile that best fits the surface brightness distribution (see Sect.2.3). This latter component is assumed to be a massless tracer sitting in the gravitational potential of the former. The stellar orbital anisotropy $\beta$, defined by Eq. (14), is assumed to be zero. Two "measurements" are made: (1) the mass $M_{\mathrm{E}}$ projected within the Einstein radius $R_{\mathrm{E}}$ (the strong lensing constraint); (2) the line-of-sight stellar velocity dispersion measured within a circular aperture of radius $1.5^{\prime \prime}$, same as used for the SLACS data (the stellar kinematics constraint). For any given power-law slope $\gamma^{\prime}$, measurement (1) constrains the normalization of the total matter density profile, with which the radial distribution of the stellar velocity dispersion can be derived by solving the spherical Jeans equation. The slope $\gamma_{0}^{\mathrm{LD}}$ (where the superscript "LD" stands for "strong lensing and dynamics" and the subscript 0 refers to the assumption of $\beta=0$ ) that results in the best fit to measurement (2) is then taken as the power-law slope of the total density profile. We searched for $\gamma_{0}^{\mathrm{LD}}$ within $[1.2,2.8]$ with a step of 0.02 . This leads to the differences between the bestfitting and the "observed" velocity dispersion in most cases smaller than $1 \mathrm{~km} / \mathrm{s}$, and in all cases no larger than $2 \mathrm{~km} / \mathrm{s}$, much smaller than the observational uncertainty (2\%-10\%).

\subsubsection{Comparisons to observations}

Fig.14 presents the total matter density slope $\gamma_{0}^{\mathrm{LD}}$ as a function of $R_{\text {eff }}$ (top panel), $\sigma_{e / 2}$ (middle panel), and $\Sigma_{*} \equiv$ $M_{*} /\left(2 \pi R_{\text {eff }}^{2}\right)$ (bottom panel). The solid and dashed lines indicate the median and the $90 \%$ boundaries of the distributions for the selected early-type galaxies between $z=0.1$ and $z=1.0$. The blue and black squares with their error bars indicate measurements for the SLACS (Auger et al. 2010b) and SL2S (Sonnenfeld et al. 2013) galaxies, respectively.

The simulation reproduces the general observational trends and scatter. The dependencies are noticeable: $\gamma_{0}^{\mathrm{LD}}$ on average decreases with increasing $R_{\text {eff }}$ but increases with increasing $\sigma_{e / 2}$ and $\Sigma_{*}$. In particular, higher-mass (higher$\left.\sigma_{e / 2}\right)$ galaxies on average have larger $\gamma_{0}^{\mathrm{LD}}$ with smaller scatter in comparison to their lower-mass (lower- $\sigma_{e / 2}$ ) counterparts. We note that, however, such a mass dependence is in part due to the correlation between the measurements of the velocity dispersion and the density slope, as well as other systematic biases (Sect. 6.1.2 and 6.1.3). In Sect.6.2, mass dependences of different slope estimators are presented and discussed.

The measurements of $\gamma_{0}^{\mathrm{LD}}$ for the simulated sample are overall shallower than observations. We note that although the selected Illustris galaxy sample shares a similar range of $\sigma_{e / 2} \in[160,400] \mathrm{km} \mathrm{s}^{-1}$ with the observed sample, they have rather different probability distributions. The former increases in number towards lower $\sigma_{e / 2}$ while the latter almost has a peak around $\sim 250 \mathrm{~km} \mathrm{~s}^{-1}$. For this reason, we present quantitative comparisons made at given $\sigma_{e / 2}$ (within a small $\sigma_{e / 2}$ range): for galaxies with $\sigma_{e / 2} \in[220,280] \mathrm{km} \mathrm{s}^{-1},\left\langle\gamma_{0}^{\mathrm{LD}}\right\rangle=1.92 \pm 0.18$ (rms) for the Illustris galaxy sample, while $\left\langle\gamma_{0}^{\mathrm{LD}}\right\rangle=2.07 \pm 0.18(\mathrm{rms})$ for
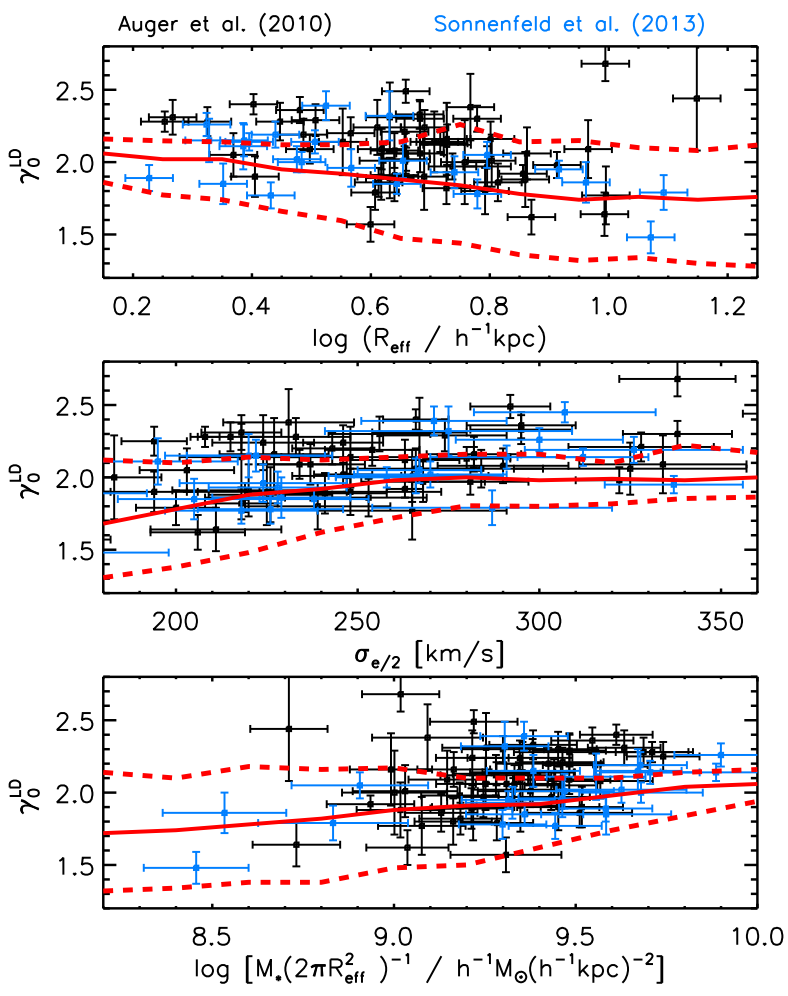

Figure 14. The total matter density slope $\gamma_{0}^{\mathrm{LD}}$ (see Sect.6.2) as a function of $R_{\text {eff }}$ (top panel), $\sigma_{e / 2}$ (middle panel), and $\Sigma_{*} \equiv M_{*} /\left(2 \pi R_{\text {eff }}^{2}\right)$ (bottom panel). The solid and the dashed lines indicate the median and the $90 \%$ boundaries of the distributions from the selected early-type galaxies at $z=0.1-1.0$. The blue and black squares show the observational results for the SLACS (Auger et al. 2010b) and SL2S (Sonnenfeld et al. 2013) samples, respectively, where the error bars indicate $1 \sigma$ error of the data.

the combined SLACS and SL2S galaxy sample. For galaxies with $\sigma_{e / 2} \in[250,300] \mathrm{km} \mathrm{s}^{-1},\left\langle\gamma_{0}^{\mathrm{LD}}\right\rangle=1.98 \pm 0.14(\mathrm{rms})$ for the Illustris sample, and $\left\langle\gamma_{0}^{\mathrm{LD}}\right\rangle=2.13 \pm 0.19$ (rms) for the observation sample.

The notably shallower $\gamma_{0}^{\mathrm{LD}}$ for the simulated galaxy sample is in fact consistent with lower central velocity dispersion $\sigma_{e / 2}$ and higher central dark matter fraction $f_{\mathrm{dm}}$ when comparing to the observational sample. This could indicate potentially inaccurate modelling of the involved baryonic physics processes in galaxy centres. We must note, however, the ad-hoc choice of a unique source redshift that was assigned to each lens redshift resulted in averagely smaller $R_{\mathrm{E}} / R_{\mathrm{eff}}$ distribution of the simulated galaxy sample compared to the observation. As a mild increase of $\gamma_{0}^{\mathrm{LD}}$ with increasing $R_{\mathrm{E}} / R_{\text {eff }}$ (i.e., steeper slopes at larger radii) was observed, such a systematic difference in $R_{\mathrm{E}} / R_{\text {eff }}$ would also cause a lower average of $\gamma_{0}^{\mathrm{LD}}$ for the simulation sample.

\subsubsection{Biases from the isotropic orbital assumption}

Combining strong lensing and single-aperture stellar kinematics to derive the total density slopes, $\beta=0$ is commonly assumed due to a lack of sufficient observational constraints. To see the effect of a non-zero anisotropy parameter $\beta$, we also calculated the total-density power-law slopes $\gamma_{\beta}^{\mathrm{LD}}$ un- 


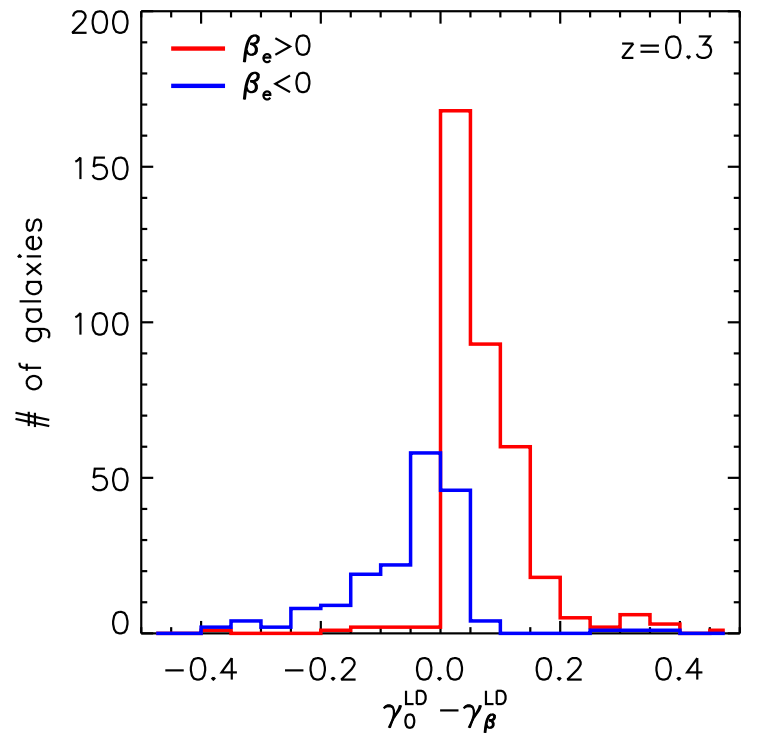

Figure 15. Histograms of $\gamma_{0}^{\mathrm{LD}}-\gamma_{\beta}^{\mathrm{LD}}$ for the selected early-type galaxy sample at $z=0.3$. Red and blue lines indicate galaxies that have $\beta>0$ and $\beta<0$ (measured within $R_{\text {eff }}$ ), respectively.

der the same assumptions as before, but using the true $\beta$ measured for the simulated galaxies. Fig.15 shows the histograms of $\gamma_{0}^{\mathrm{LD}}-\gamma_{\beta}^{\mathrm{LD}}$ for the selected early-type galaxy sample at $z=0.3$. Red and blue lines indicate galaxies that have $\beta>0$ and $\beta<0$ (measured within $R_{\text {eff }}$ ), respectively. The former distribution (for radially anisotropic galaxies) peaks at $\gamma_{0}^{\mathrm{LD}}-\gamma_{\beta}^{\mathrm{LD}}>0$, while the latter (for tangentially anisotropic galaxies) peaks at $\gamma_{0}^{\mathrm{LD}}-\gamma_{\beta}^{\mathrm{LD}}<0$. This demonstrates that the true slopes of radially anisotropic systems tend to be overestimated assuming $\beta=0$, while those of tangential ones tend to be underestimated (see also Koopmans $2006 ; 2009)$.

It is worth noting that, as shown in Fig. 7 and Fig. 9, $\beta$ markedly depends on stellar velocity dispersion and also evolves with redshift. This leads to possible systematic biases of the observational measurements. The total density slopes of higher- (lower-) mass galaxies or galaxies at lower(higher-) redshifts are likely to be overestimated (underestimated) by $\gamma_{0}^{\mathrm{LD}}$ when assuming $\beta=0$.

\subsubsection{Robustness of the power-law assumption}

As shown by Xu et al. (2016), true profiles of realistic galaxies deviate from power-law distributions. In order to validate the robustness of the applied power-law assumption despite this fact, we first defined a curvature parameter for the 3-D enclosed mass distribution $M(r)$ (defined by Eq. (18)) between $r_{1}$ and $r_{2}$ :

$$
\xi_{M_{3 \mathrm{~d}}}\left(r_{1}, r_{2}\right) \equiv \frac{M\left(\sqrt{r_{1} r_{2}}\right)}{\sqrt{M\left(r_{1}\right) M\left(r_{2}\right)}} .
$$

This curvature parameter $\xi_{M_{3 \mathrm{~d}}}\left(r_{1}, r_{2}\right)$ directly quantifies the closeness of $M(r)$, and thus $\rho(r)$, to a power-law distribution between $r_{1}$ and $r_{2}$. If the deviation of $\xi_{M_{3 \mathrm{~d}}}\left(r_{1}, r_{2}\right)$ from unity is large, then the power-law approximation is poor. We set $\left[r_{1}, r_{2}\right]$ to be $[0.5,2.0] R_{\mathrm{E}}$, which is the most relevant radial range for approaches that combine strong lensing and stellar kinematics.

We calculated $\xi_{M_{3 \mathrm{~d}}}\left(0.5 R_{\mathrm{E}}, 2.0 R_{\mathrm{E}}\right)$ for all the selected early-type galaxies from the simulation. Its dependence on $\sigma_{e / 2}$ is presented in the left-most panel of Fig.[16] The crosses indicate the simulation results at $z=0.3$, the solid and the dashed lines indicate the median and the $90 \%$ boundaries of this distribution. In fact, both $\xi_{M_{3 \mathrm{~d}}}\left(0.5 R_{\mathrm{E}}, 2.0 R_{\mathrm{E}}\right)$ and $\xi_{M_{3 \mathrm{~d}}}\left(0.5 R_{\text {eff }}, 2.0 R_{\text {eff }}\right)$ show decreasing trends towards larger systems. In this case, the power-law approximation becomes worse for galaxies with central velocity dispersion $\sigma_{e / 2} \lesssim 250 \mathrm{~km} \mathrm{~s}^{-1}$. As shown by Xu et al. (2016), this leads to significantly biased determinations of the Hubble constant $H_{0}$ when power-law mass models are used to describe the lensing galaxies as they artificially break the so-called "mass sheet degeneracy" (e.g., Falco et al. 1985; Schneider \& Sluse 2013).

The breakdown of the power-law approximation can also lead to biased estimates of other derived quantities, e.g., the $2-\mathrm{D}$ enclosed mass distribution $M_{2 \mathrm{~d}}(\leqslant R)$ and the projected dark matter fraction $f_{\mathrm{dm}}(\leqslant R)$, if they were derived assuming a power-law total density profile with slope $\gamma_{0}^{\mathrm{LD}}$ (for $f_{\mathrm{dm}}$, the dark matter mass is obtained by subtracting the observationally-constrained stellar mass from the total mass projected within a given aperture). In order to identify potential biases in these quantities, we further calculate two ratios: $(1)$ between $M_{2 \mathrm{~d}}^{\mathrm{PL}}(\leqslant R)$ that is derived under the power-law assumption and the true mass distribution $M_{2 \mathrm{~d}}^{\text {true }}(\leqslant R)$; and $(2)$ between $f_{\mathrm{dm}}^{\mathrm{PL}}(\leqslant R)$ that is derived under the power-law assumption and the true fraction $f_{\mathrm{dm}}^{\text {true }}(\leqslant R)$. The "true" quantities are directly measured for simulated galaxies. Note that by construction, $M_{2 \mathrm{~d}}^{\mathrm{PL}}\left(\leqslant R_{\mathrm{E}}\right)=M_{2 \mathrm{~d}}^{\text {true }}\left(\leqslant R_{\mathrm{E}}\right)$. However, this is not necessarily the case at other radii, unless the true distribution is indeed a power law.

We measured the two ratios within different aperture radii for the selected early-type Illustris galaxies. In Fig.16. the middle and right-most panels show the enclosed mass ratio $M_{2 \mathrm{~d}}^{\mathrm{PL}} / M_{2 \mathrm{~d}}^{\text {true }}$ versus $\sigma_{e / 2}$, and the projected dark matter fraction ratio $f_{\mathrm{dm}}^{\mathrm{PL}} / f_{\mathrm{dm}}^{\text {true }}$ versus $\sigma_{e / 2}$, respectively (for the same galaxy sample at $z=0.3$ ). We note that the ratios deviate from unity due to a combination of two effects: (1) poor approximations of the power-law models for lower-mass galaxies; and (2) biased (power-law) slope estimates due to single-aperture kinematics data and the stellar isotropy assumption. These combined effects could lead to significantly biased estimates of the enclosed mass and the projected dark matter fraction, especially when contraints are made for lower-mass galaxies. The deviations from unity as well as the scatter also increase with redshift up to $z=1.0$.

\subsection{Different estimators of the total density slopes}

To investigate the variations among different total-slope estimators, we calculated several sets of slopes for the simulated galaxies within different radial ranges, namely, the simple power-law dynamical slopes $\gamma_{0}^{\mathrm{LD}}$ and $\gamma_{\beta}^{\mathrm{LD}}$, the mass-weighted slopes $\gamma^{\mathrm{MW}}\left(R_{\text {eff }}\right)$ and $\gamma^{\mathrm{MW}}\left(2.0 R_{\text {eff }}\right)$, the power-law fitted slopes $\gamma^{\mathrm{PL}}\left(0.5 R_{\text {eff }}, R_{\text {eff }}\right)$ and $\gamma^{\mathrm{PL}}\left(0.5 R_{\text {eff }}, 2.0 R_{\text {eff }}\right)$, and finally the average slopes $\gamma^{\mathrm{AV}}\left(0.5 R_{\text {eff }}, R_{\text {eff }}\right)$ and $\gamma^{\mathrm{AV}}\left(0.5 R_{\text {eff }}, 2.0 R_{\text {eff }}\right)$.

Table 1 presents the stellar mass-weighted mean and 

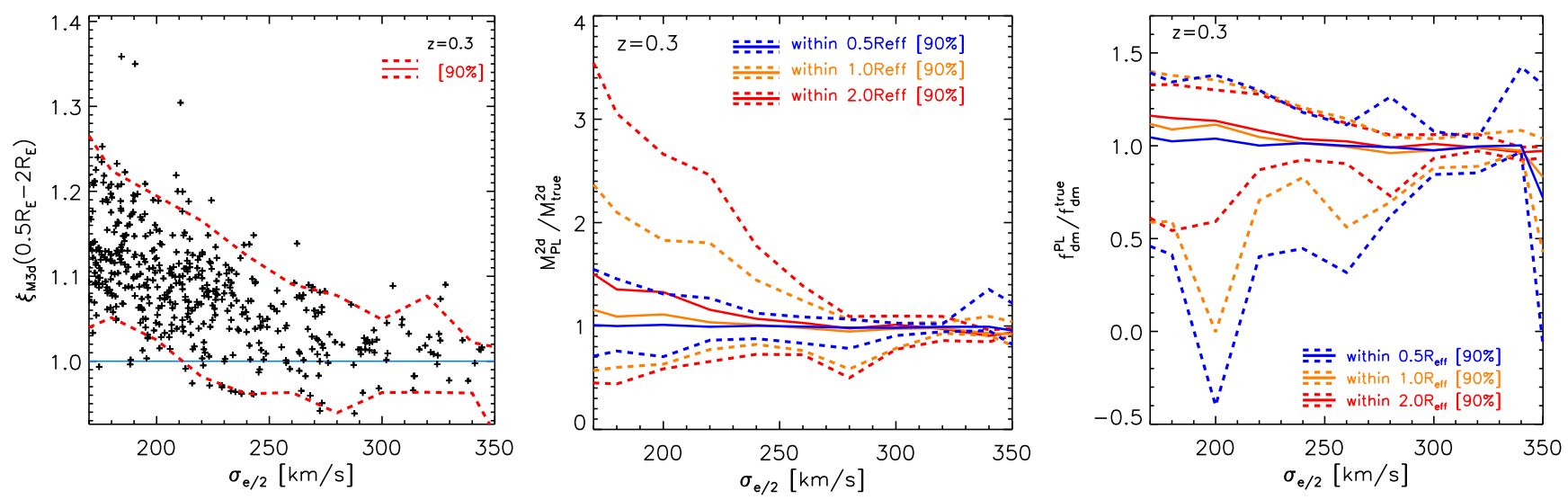

Figure 16. The curvature parameter $\xi_{M_{3 \mathrm{~d}}}\left(0.5 R_{\mathrm{E}}, 2.0 R_{\mathrm{E}}\right)$ (left panel), the enclosed mass ratio $M_{2 \mathrm{~d}}^{\mathrm{PL}} / M_{2 \mathrm{~d}}^{\text {true }}$ (middle panel) and the projected dark matter fraction $f_{\mathrm{dm}}^{\mathrm{PL}} / f_{\mathrm{dm}}^{\text {true }}$ (right panel), as a function of the central stellar velocity dispersion $\sigma_{e} / 2$ for the early-type galaxy sample at $z=0.3$. In all three panels, the solid and the dashed lines indicate the median and the $90 \%$ boundaries of the distributions. The blue, red and orange curves present measurements made within different aperture sizes as specified in the panels. Note that the median values of the ratios measured within $0.5 R_{\text {eff }}$ are close to 1.0 , this is because the simulation sample has on average $\left\langle R_{\mathrm{E}} / R_{\text {eff }}\right\rangle \sim 0.5$, and by construction $M_{2 \mathrm{~d}}^{\mathrm{PL}}\left(R \leqslant R_{\mathrm{E}}\right)=M_{2 \mathrm{~d}}^{\mathrm{true}}\left(R \leqslant R_{\mathrm{E}}\right)$. In addition, a small fraction of galaxies have negative $f_{\mathrm{dm}}^{\mathrm{PL}}$ (as can be seen from the right panel), which originates in the power-law assumption. For these galaxies, the power-law model that best fits both the strong lensing and kinematical constraints predicts total masses smaller than the stellar masses within the studied aperture.

Table 1. Stellar mass-weighted mean and standard deviation of the density slopes for the selected early-type galaxy sample at $z=0.3$

\begin{tabular}{l|c|c}
\hline slope estimator & mean & standard deviation \\
\hline$\gamma_{0}^{\mathrm{LD}}$ & 1.83 & 0.24 \\
$\gamma_{\beta}^{\mathrm{LD}}$ & 1.80 & 0.23 \\
$\gamma^{\mathrm{MW}}\left(R_{\text {eff }}\right)$ & 1.95 & 0.14 \\
$\gamma^{\mathrm{MW}}\left(2.0 R_{\text {eff }}\right)$ & 1.99 & 0.14 \\
$\gamma^{\mathrm{PL}}\left(0.5 R_{\text {eff }}, R_{\text {eff }}\right)$ & 2.08 & 0.27 \\
$\gamma^{\mathrm{PL}}\left(0.5 R_{\text {eff }}, 2.0 R_{\text {eff }}\right)$ & 2.07 & 0.26 \\
$\gamma^{\mathrm{AV}}\left(0.5 R_{\text {eff }}, R_{\text {eff }}\right)$ & 2.08 & 0.26 \\
$\gamma^{\mathrm{AV}}\left(0.5 R_{\text {eff }}, 2.0 R_{\text {eff }}\right)$ & 2.06 & 0.22 \\
\hline$\gamma_{\mathrm{dm}}^{\mathrm{PL}}\left(0.5 R_{\text {eff }}, R_{\text {eff }}\right)$ & 1.49 & 0.22 \\
$\gamma_{\mathrm{dm}}^{\mathrm{PL}}\left(0.5 R_{\text {eff }}, 2.0 R_{\text {eff }}\right)$ & 1.58 & 0.17 \\
$\gamma_{*}^{\mathrm{PL}}\left(0.5 R_{\text {eff }}, R_{\text {eff }}\right)$ & 2.74 & 0.30 \\
$\gamma_{*}^{\mathrm{PL}}\left(0.5 R_{\text {eff }}, 2.0 R_{\text {eff }}\right)$ & 2.87 & 0.26 \\
\hline
\end{tabular}

standard deviation of each above-mentioned slope estimator for the selected early-type galaxy sample at $z=0.3$. In Fig.17 we plot, in particular, four typical slopes measured around and within $R_{\text {eff }}$, for the same galaxy sample, as a function of $\sigma_{e / 2}$. The red, orange, green and blue curves indicate the distributions of $\gamma_{0}^{\mathrm{LD}}, \gamma_{\beta}^{\mathrm{LD}}, \gamma^{\mathrm{MW}}\left(R_{\text {eff }}\right)$ and $\gamma^{\mathrm{PL}}\left(0.5 R_{\text {eff }}, R_{\text {eff }}\right)$, respectively. The solid and the dashed line styles show the median and the $68 \%$ range of each distribution, respectively. The thicker dashed and dotted lines present the best linear fits to the data.

We see that within a large fraction of the velocity range investigated here, the median slopes are all about isothermal. In particular, the median and scatter of the intrinsic density slope estimators $\gamma^{\mathrm{PL}}$ and $\gamma^{\mathrm{AV}}$ are consistent with the observational results from 2-D kinematical data (e.g., Barnabè et al. 2011; Cappellari et al. 2015).

Interestingly, systematic discrepancies are seen among different slope estimators. For most galaxies, since the local slope $\gamma(r)$ (defined by Eq. (17)) tends to decrease, i.e., the profile turning flatter, towards smaller $r$ (see also Dutton \& Treu 2014 for more discussion), one finds $\gamma^{\mathrm{MW}}\left(R_{\mathrm{eff}}\right)<\gamma^{\mathrm{PL}}\left(0.5 R_{\text {eff }}, R_{\text {eff }}\right)$ as expected. Between the two simple power-law dynamical slopes, the fact that on average $\gamma_{0}^{\mathrm{LD}}>\gamma_{\beta}^{\mathrm{LD}}$ (within a large span of $\sigma_{e / 2}$ ) can be explained by the stellar orbital anisotropies, because the majority of the galaxy sample has radial anisotropies (as can be seen from Fig.(7). For galaxies with $\sigma_{e / 2} \lesssim 250 \mathrm{~km} \mathrm{~s}^{-1}$, a moderate disagreement exists between the simple dynamical slope $\gamma^{\mathrm{LD}}$ and the intrinsic slope $\gamma^{\mathrm{MW}}\left(R_{\text {eff }}\right)$. This difference increases with redshift and can be attributed to the poor approximations of the power-law models for lower-mass galaxies.

Specifically, as can be seen from Fig.16 the curvature parameter fulfils $\xi_{M_{3 \mathrm{~d}}}\left(0.5 R_{\mathrm{E}}, 2.0 R_{\mathrm{E}}\right)>1.0$ for the majority of lower-mass galaxies. Mathematically, $\xi_{M_{3 \mathrm{~d}}}>1.0$ means that, to first-order approximation, $M(r)$ is concaveupward, lying above the power-law interpolation between the two radii. The local mass slope $\frac{\mathrm{d} \ln M(r)}{\mathrm{d} \ln r}$ therefore decreases with increasing $r$, which according to Eq. (16) corresponds to an increase of $\gamma_{\mathrm{tot}}^{\mathrm{MW}}(r)$ within the same radial range. For galaxies with $\xi_{M_{3 \mathrm{~d}}}>1.0\left(\xi_{M_{3 \mathrm{~d}}}<1.0\right)$, the larger the radius $r$, the larger (smaller) the slope $\gamma^{\mathrm{MW}}(r)$.

As pointed out by Dutton \& Treu (2014), for a perfect power-law distribution, the density slopes $\gamma^{\mathrm{LD}}$ that are derived under the power-law assumption are essentially the same as $\gamma^{\mathrm{MW}}$. For a realistic galaxy, as the power-law model could be a poor approximation, this, however, is not necessarily the case. $\gamma^{\mathrm{LD}}$ rather measures some averaged $\gamma_{\text {tot }}^{\mathrm{MW}}(r)$ between $0.5 R_{\mathrm{E}}$ and $2.0 R_{\mathrm{E}}$. As $\xi_{M_{3 \mathrm{~d}}}>1.0$ holds for the majority of lower-mass galaxies, $\gamma^{\mathrm{MW}}\left(0.5 R_{\mathrm{E}}\right) \lesssim \gamma^{\mathrm{LD}} \lesssim \gamma^{\mathrm{MW}}\left(2.0 R_{\mathrm{E}}\right)$ can therefore be in general expected. For these galaxies, we also found an average radius ratio of $\left\langle R_{\text {eff }} / R_{\mathrm{E}}\right\rangle \sim 2.0$, which eventually led to the general trend of $\gamma^{\mathrm{LD}} \lesssim \gamma^{\mathrm{MW}}\left(R_{\text {eff }}\right)$ seen for galaxies with $\sigma_{e / 2} \lesssim 250 \mathrm{~km} \mathrm{~s}^{-1}$.

It is also interesting to note that different slope esti- 


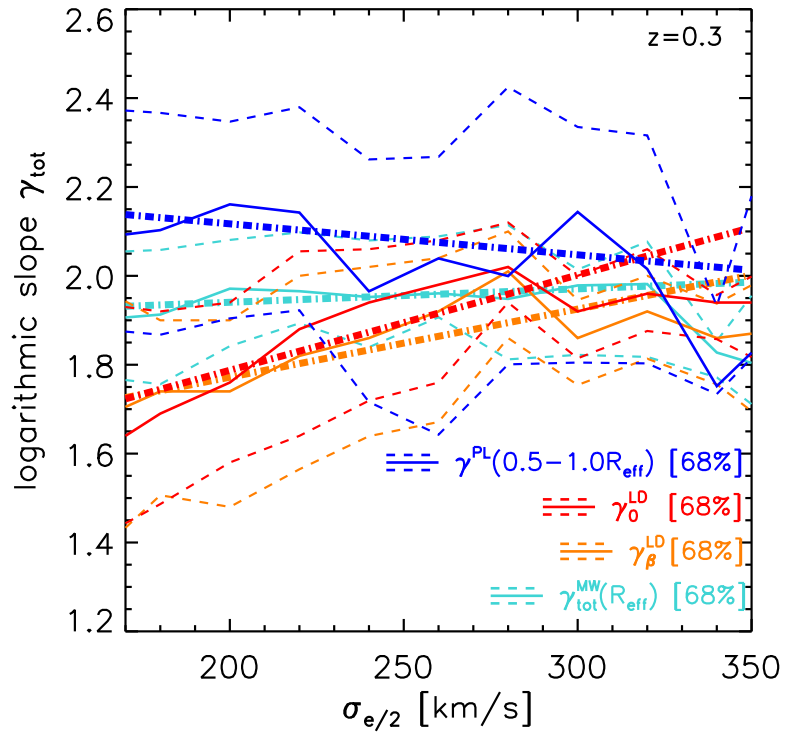

Figure 17. Total density slopes measured using different methods, as a function of the central stellar velocity dispersion $\sigma_{e / 2}$, for the selected early-type galaxy sample at $z=0.3$. The red, orange, green and blue curves indicate the distributions of $\gamma_{0}^{\mathrm{LD}}, \gamma_{\beta}^{\mathrm{LD}}, \gamma^{\mathrm{MW}}\left(R_{\text {eff }}\right)$ and $\gamma^{\mathrm{PL}}\left(0.5 R_{\text {eff }}, R_{\text {eff }}\right)$, respectively. The solid and the dashed line styles show the median and the $68 \%$ boundaries of each distribution, respectively. The thicker dashed and dotted lines present the best linear fits to the data. Linear regression of $\gamma$ as a function of $\sigma_{e / 2}$ resulted in $\partial \gamma_{0}^{\mathrm{LD}} / \partial \sigma_{e / 2}=0.0021 \pm 0.0002$ with a linear correlation coefficient $r=0.36 ; \partial \gamma_{\beta}^{\mathrm{LD}} / \partial \sigma_{e / 2}=0.0015 \pm 0.0002$ with $r=$ $0.27 ; \partial \gamma^{\mathrm{MW}}\left(R_{\mathrm{eff}}\right) / \partial \sigma_{e / 2}=0.0003 \pm 0.0001$ with $r=0.10$ and $\partial \gamma_{\text {tot }}^{\mathrm{PL}}\left(0.5 R_{\mathrm{eff}}, R_{\mathrm{eff}}\right) / \partial \sigma_{e / 2}=-0.0007 \pm 0.0003$ with $r=-0.11$.

mators have different mass dependences. As both the stellar anisotropy $\beta$ and the profile curvature $\xi_{\mathrm{M}_{3 \mathrm{~d}}}$ depend on mass, the assumptions of isotropic orbits and/or power-law profiles affect the mass dependence of the lensing and dynamic slope $\gamma^{\mathrm{LD}}$. Specifically, for the early-type galaxies selected at $z=0.3$, linear regression of $\gamma^{\mathrm{LD}}$ as a function of $\sigma_{e / 2}$ resulted in $\partial \gamma_{0}^{\mathrm{LD}} / \partial \sigma_{e / 2}=0.0021 \pm 0.0002$ with a linear correlation coefficient $r=0.36$; and $\partial \gamma_{\beta}^{\mathrm{LD}} / \partial \sigma_{e / 2}=0.0015 \pm 0.0002$ with $r=0.27$, indicating positive correlations with $\sigma_{e / 2}$.

On the contrary, the true density slope estimator $\gamma^{\mathrm{PL}}$ shows decreasing trends with increasing $\sigma_{e / 2}$ (see also, e.g., Humphrey \& Buote 2010; Remus et al. 2013, 2016). In particular, for the galaxy sample at $z=$ 0.3 , linear regression of $\gamma^{\mathrm{PL}}$ as a function of $\sigma_{e / 2}$ resulted in $\partial \gamma_{\text {tot }}^{\mathrm{PL}}\left(0.5 R_{\mathrm{eff}}, R_{\mathrm{eff}}\right) / \partial \sigma_{e / 2}=-0.0007 \pm 0.0003$ with a linear correlation coefficient $r=-0.11$; and $\partial \gamma^{\mathrm{PL}}\left(0.5 R_{\text {eff }}, 2.0 R_{\text {eff }}\right) / \partial \sigma_{e / 2}=-0.0010 \pm 0.0002$ with $r=$ -0.18 . We also note that such dependences on $\sigma_{e / 2}$ were observed across all the redshifts studied here.

Relating the anti-correlation between $\gamma^{\mathrm{PL}}$ and $\sigma_{e / 2}$ with previously studied $\sigma_{e / 2}$ dependences (see Sect.4), we see that lower-mass galaxies have higher central gas fractions, bluer colours, more tangentially anisotropic orbits and slightly steeper inner slopes than their more massive counterparts.

\subsection{Inner slope estimates of the dark matter and the stellar distributions}

In centres of galaxies, both dark matter and stellar components shape the total density profile, which approximately follows an isothermal distribution. In order to see how much the slopes of individual components deviate from isothermal, and in particular, how much the NFW profile is modified due to the presence of baryons, we calculated $\gamma_{\mathrm{dm}}^{\mathrm{PL}}\left(0.5 R_{\mathrm{eff}}, R_{\mathrm{eff}}\right)$ and $\gamma_{*}^{\mathrm{PL}}\left(0.5 R_{\text {eff }}, R_{\text {eff }}\right)$ for the simulated galaxies. Table 1 reports the stellar mass-weighted mean and standard deviation of the stellar and dark matter slopes for the selected early-type galaxy sample at $z=0.3$.

Their dependences on the central stellar velocity dispersion $\sigma_{e / 2}$ are shown in Fig.18, where the solid and the dashed lines indicate the median and the $90 \%$ boundaries of either distribution, respectively; and the thicker dashed and dotted lines present the best linear fits to the data. Linear regression of $\gamma_{\mathrm{dm}}$ and $\gamma_{*}$ as functions of $\sigma_{e / 2}$ resulted in $\partial \gamma_{\mathrm{dm}}^{\mathrm{PL}}\left(0.5 R_{\text {eff }}, R_{\text {eff }}\right) / \partial \sigma_{e / 2}=$ $-0.0004 \pm 0.0002$ with a linear correlation coefficient $r=-0.06 ; \partial \gamma_{\mathrm{dm}}^{\mathrm{PL}}\left(0.5 R_{\mathrm{eff}}, 2.0 R_{\mathrm{eff}}\right) / \partial \sigma_{e / 2}=-0.0009 \pm$ 0.0002 with $r=-0.22$ for the dark matter density slopes, and $\partial \gamma_{*}^{\mathrm{PL}}\left(0.5 R_{\text {eff }}, R_{\text {eff }}\right) / \partial \sigma_{e / 2}=-0.0002 \pm$ 0.0003 with a linear correlation coefficient $r=-0.03$; $\partial \gamma_{*}^{\mathrm{PL}}\left(0.5 R_{\text {eff }}, 2.0 R_{\text {eff }}\right) / \partial \sigma_{e / 2}=-0.0004 \pm 0.0002$ with $r=$ -0.07 for the stellar density slopes.

In addition, the stellar component, with a mean slope of $\left\langle\gamma_{*}^{\mathrm{PL}}\left(0.5 R_{\text {eff }}, R_{\text {eff }}\right)\right\rangle \sim 2.74$, is significantly steeper than the total matter distribution (for which $\left\langle\gamma^{\mathrm{PL}}\left(0.5 R_{\text {eff }}, R_{\text {eff }}\right)\right\rangle \sim$ $2.08)$. The inner dark matter slope is notably steeper than the predicted logarithmic slope of unity for an NFW profile at small radii. A mean slope of $\left\langle\gamma_{\mathrm{dm}}^{\mathrm{PL}}\left(0.5 R_{\text {eff }}, R_{\text {eff }}\right)\right\rangle \sim 1.49$ is well consistent with both observations (e.g., Sonnenfeld et al. 2012; Cappellari et al. 2013; Oguri et al. 2014; Bruderer et al. 2016) and published simulations (e.g., Johansson et al. 2012; Remus et al. 2013).

\subsection{Evolutionary trends}

Fig.19 shows the cosmic evolution of the matter density slopes measured for the selected early-type galaxies from the Illustris simulation. Again we applied linear regression to the slopes studied above as functions of redshift $z$. The best linear fit resulted in $\partial \gamma_{\mathrm{dm}}^{\mathrm{PL}}\left(0.5 R_{\mathrm{eff}}, R_{\mathrm{eff}}\right) / \partial z=0.18 \pm 0.01$ with a correlation coefficient $r=0.20$ for the dark matter slope evolution; $\partial \gamma_{*}^{\mathrm{PL}}\left(0.5 R_{\text {eff }}, R_{\text {eff }}\right) / \partial z=0.28 \pm 0.02$ with $r=0.20$ for the stellar slope evolution. Both become shallower with decreasing redshift.

For the total matter density slopes, $\partial \gamma_{0}^{\mathrm{LD}} / \partial z=-0.03 \pm$ 0.01 with $r=-0.03 ; \partial \gamma_{\beta}^{\mathrm{LD}} / \partial z=0.14 \pm 0.01$ with $r=$ $0.15 ; \partial \gamma^{\mathrm{MW}}\left(R_{\mathrm{eff}}\right) / \partial z=0.12 \pm 0.01$ with $r=0.25$ and $\partial \gamma^{\mathrm{PL}}\left(0.5 R_{\text {eff }}, R_{\text {eff }}\right) / \partial z=0.11 \pm 0.01$ with $r=0.11$. As can be seen, most slope estimators also indicate a shallower trend towards lower redshifts. In particular, the mean magnitude and evolutionary trend of $\gamma^{\mathrm{PL}}$ found for the earlytype Illustris galaxies are consistent with results from previous cosmological simulations and theoretical studies (e.g., Johansson et al. 2012; Remus et al. 2013, 2016; Sonnenfeld et al. 2014).

It is noteworthy to point out that unlike the redshift evolution of the intrinsic slope estimators, a mild increase 


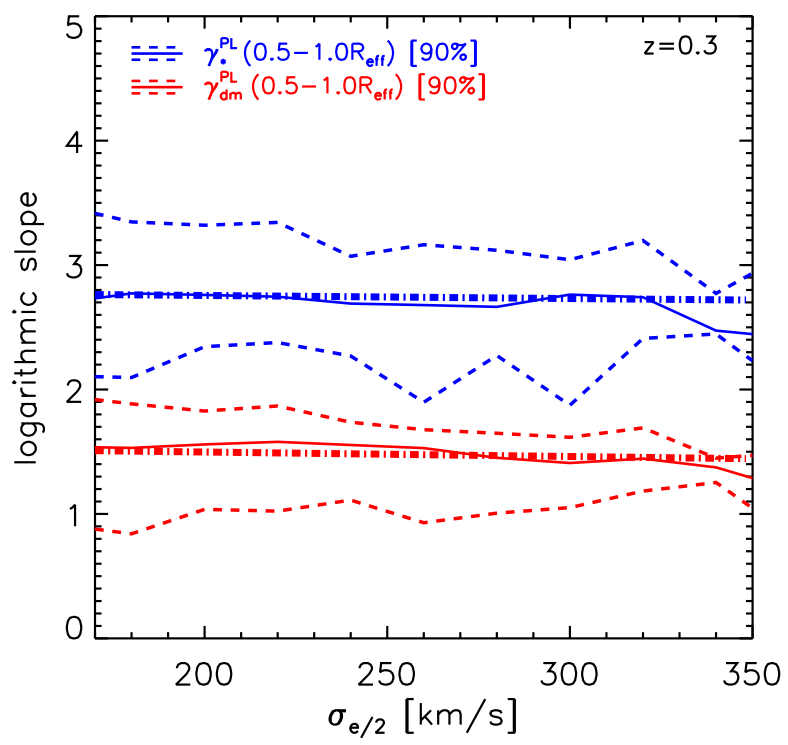

Figure 18. The power-law fitted slopes of the inner dark matter distribution $\gamma_{\mathrm{dm}}^{\mathrm{PL}}\left(0.5 R_{\mathrm{eff}}, R_{\mathrm{eff}}\right)$ (red) and of the inner stellar distribution $\gamma_{*}^{\mathrm{PL}}\left(0.5 R_{\mathrm{eff}}, R_{\mathrm{eff}}\right)$ (blue), as a function of the central stellar velocity dispersion $\sigma_{e / 2}$, for the selected early-type galaxy sample at $z=0.3$. The solid and the dashed line styles indicate the median and the $90 \%$ boundaries of either distribution, respectively. The thicker dashed and dotted lines present the best linear fits to the data. Linear regression of $\gamma_{\mathrm{dm}}$ and $\gamma_{*}$ as functions of $\sigma_{e / 2}$ resulted in $\partial \gamma_{\mathrm{dm}}^{\mathrm{PL}}\left(0.5 R_{\mathrm{eff}}, R_{\mathrm{eff}}\right) / \partial \sigma_{e / 2}=$ $-0.0004 \pm 0.0002$ with a linear correlation coefficient $r=$ $-0.06 ; \partial \gamma_{\mathrm{dm}}^{\mathrm{PL}}\left(0.5 R_{\mathrm{eff}}, 2.0 R_{\mathrm{eff}}\right) / \partial \sigma_{e / 2}=-0.0009 \pm 0.0002$ with $r=-0.22$ for the dark matter density slopes, and $\partial \gamma_{*}^{\mathrm{PL}}\left(0.5 R_{\text {eff }}, R_{\text {eff }}\right) / \partial \sigma_{e / 2}=-0.0002 \pm 0.0003$ with a linear correlation coefficient $r=-0.03 ; \partial \gamma_{*}^{\mathrm{PL}}\left(0.5 R_{\text {eff }}, 2.0 R_{\text {eff }}\right) / \partial \sigma_{e / 2}=$ $-0.0004 \pm 0.0002$ with $r=-0.07$ for the stellar density slopes.

of $\gamma_{0}^{\mathrm{LD}}$ was seen at lower redshifts for the early-type Illustris galaxies. Interestingly, this is roughly consistent with strong lensing observations. Currently, the only observationally constrained redshift evolution (up to $z=1.0$ ) comes from combining strong lensing techniques with singleaperture stellar kinematics. The derived slopes $\gamma_{0}^{\mathrm{LD}}$ for the SLACS, SL2S and BELLS galaxy samples consistently show a mild steepening towards lower redshifts (e.g., Koopmans et al. 2006; Auger et al. 2010b; Ruff et al. 2011; Bolton et al. 2012; Sonnenfeld et al. 2013, 2015). We stress that such an apparent evolutionary trend of $\gamma_{0}^{\mathrm{LD}}$ may not represent the true evolution of the total central density slopes of early-type galaxies, because the derivation involves various model assumptions (e.g., spherical symmetry, isotropic stellar orbits and power-law distributions) and thus suffers from systematic biases (see Sect.6.1.2, 6.1.3 and Fig.19).

On the other hand, we also caution that $\gamma_{0}^{\mathrm{LD}}$ derived for the simulated sample cannot be directly compared to the observational results due to sampling bias. In particular, the simulation sample and the observational sample have different probability distributions for $\sigma_{e / 2}$ and (normalized) $R_{\mathrm{E}}$ in such a way that the former sample would experience a relatively lower mean of $\gamma_{0}^{\mathrm{LD}}$ because of the existence of a larger fraction of lower-mass systems and/or systems with smaller (normalized) $R_{\mathrm{E}}$. A fair comparison between the simulation and observations would require (1) strictly adopting observational criteria to select simulation samples, and (2) 2-D kinematics data and high-resolution imaging data for a large number of galaxies out to high redshifts. This could be facilitated by future Integral-Field-Unit deep surveys.

\section{CONCLUSIONS AND DISCUSSION}

In this work, we used the highest resolution run of the Illustris simulation project (Vogelsberger et al. 2014a, b; Genel et al. 2014; Sijacki et al. 2015; Nelson et al. 2015) to study the stellar orbital anisotropies, the central dark matter fractions and the central radial density slopes of early-type galaxies from $z=1.0$ to the present. The early-type galaxies were identified according to their radial surface brightness distributions (in the SDSS $g, r$ and $i$ bands) as those systems which can be better fit by de Vaucouleurs profiles than by exponential profiles (see Sect. 2.4).

In particular, we selected central early-types galaxies with stellar velocity dispersions within an observationally motivated range equal to $\sigma_{e / 2} \in[160,400] \mathrm{kms}^{-1}$ (e.g., Bolton et al. 2008a). The resulting galaxy sample roughly reproduces the observed mass-size-velocity dispersion relations (see Sect.3.2). A variety of galaxy properties of this simulation sample were compared to observed early-type galaxies from existing galaxy surveys and strong lensing surveys. Below we summarize the main findings of our analysis:

- Towards galaxy centres, baryons dominate the shape of the total matter distribution. The ratio between the ellipticities of the luminous and the total distributions is on average $(b / a)_{\text {gal }} /(b / a)_{\text {tot }} \sim 0.9$, measured within half of the effective radius. As the radius increases, the total matter distribution becomes rounder and this ratio becomes smaller. The misalignment angle $\Delta \phi_{\mathrm{RA}}$ between the luminous and the total matter distributions is consistent with zero and shows a standard deviation of $\lesssim 10$ degree. In general, the inner regions of low-redshift galaxies show better alignment between the luminous and the total matter distributions than higher-redshift galaxies (see Sect. 3.1).

- We measured the velocity anisotropy parameter $\beta$ as defined in Eq. (14) for the simulated early-type galaxies, assuming spherically symmetric density distributions. We found that higher-mass galaxies and galaxies at lower redshifts tend to have more radially anisotropic stellar orbits $(\beta>0)$ compared to their lower-mass and higher-redshift counterparts, consistent with their redder $B-V$ colours and smaller central (cold) gas fractions, which indicates the existence of relatively old stellar populations that are primarily passively evolving (see Sect. 4).

- We measured the projected dark matter fraction $f_{\mathrm{dm}}$ within $5 \mathrm{kpc}$ from the galaxy centres to be $40 \%-50 \%$ for the Illustris early-type galaxies. This range is noticeably higher than suggested by measurements from the SLACS and SL2S surveys. The projected dark matter fractions measured within the effective radius $R_{\text {eff }}$ show a very mild dependence on the central velocity dispersion $\sigma_{e / 2}$, but a clear positive correlation with $\lg R_{\text {eff }}$. The latter could be an aperture effect due to increasing $f_{\mathrm{dm}}$ at larger radii (see Sect. 5).

- We applied a common technique used observationally 

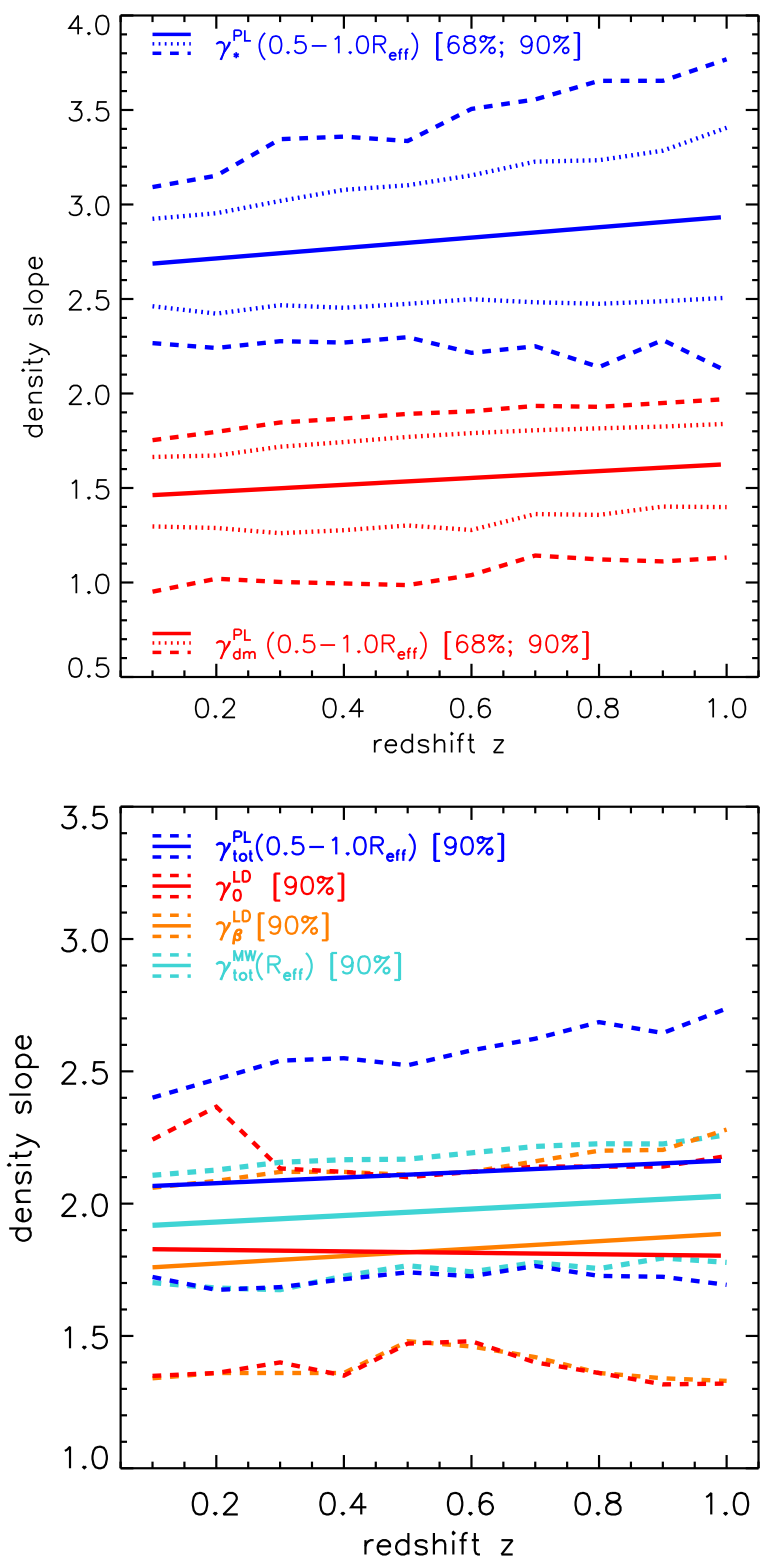

Figure 19. The cosmic evolution of the matter density slopes measured for the selected early-type galaxies from the Illustris simulation. Top panel: the red and blue curves present the slopes, $\gamma_{\mathrm{dm}}^{\mathrm{PL}}\left(0.5 R_{\text {eff }}, R_{\text {eff }}\right)$ and $\gamma_{*}^{\mathrm{PL}}\left(0.5 R_{\text {eff }}, R_{\text {eff }}\right)$, of the dark matter and stellar distributions, respectively. Bottom panel: the red, orange, green and blue curves give the slopes of $\gamma_{0}^{\mathrm{LD}}, \gamma_{\beta}^{\mathrm{LD}}$, $\gamma^{\mathrm{MW}}\left(R_{\text {eff }}\right)$ and $\gamma^{\mathrm{PL}}\left(0.5 R_{\text {eff }}, R_{\text {eff }}\right)$, respectively. The solid, dotted and dashed lines show the best linear fit to the data, the $68 \%$ and $90 \%$ boundaries of the distributions, respectively. Note that linear regression resulted in $\partial \gamma_{\mathrm{dm}}^{\mathrm{PL}}\left(0.5 R_{\mathrm{eff}}, R_{\mathrm{eff}}\right) / \partial z=0.18 \pm 0.01$ with a correlation coefficient $r=0.20$ for the dark matter slope evolution; $\partial \gamma_{*}^{\mathrm{PL}}\left(0.5 R_{\text {eff }}, R_{\text {eff }}\right) / \partial z=0.28 \pm 0.02$ with $r=0.20$ for the stellar slope evolution. For the total matter density slopes, $\partial \gamma_{0}^{\mathrm{LD}} / \partial z=-0.03 \pm 0.01$ with $r=-0.03 ; \partial \gamma_{\beta}^{\mathrm{LD}} / \partial z=0.14 \pm 0.01$ with $r=0.15 ; \partial \gamma^{\mathrm{MW}}\left(R_{\text {eff }}\right) / \partial z=0.12 \pm 0.01$ with $r=0.25$ and $\partial \gamma^{\mathrm{PL}}\left(0.5 R_{\mathrm{eff}}, R_{\mathrm{eff}}\right) / \partial z=0.11 \pm 0.01$ with $r=0.11$. that combines strong lensing with single-aperture kinematics to constrain the total (central) density slopes $\gamma_{0}^{\mathrm{LD}}$ of the simulated galaxies. The inferred $\gamma_{0}^{\mathrm{LD}}$ is on average shallower than in observations, for which the mean distribution is much closer to isothermal (see Auger et al. 2010b, Sonnenfeld et al. 2013). However, the simulation roughly reproduces the observed dependences of $\gamma_{0}^{\mathrm{LD}}$ on other galaxy properties (e.g., effective radius, stellar velocity dispersion, and surface density, see Sect.6.1.1).

- The slope $\gamma_{0}^{\mathrm{LD}}$ derived using the above-mentioned simple practical technique suffers from systematic biases due to two major assumptions, i.e., the isotropic stellar orbits and the power-law density model. As a result, (1) radially (tangentially) anisotropic orbits cause $\gamma_{0}^{\mathrm{LD}}$ to overestimate (underestimate) the true density slopes (see Sect.6.1.2); and (2) the poor approximation of the power-law assumption causes $\gamma_{0}^{\mathrm{LD}}$ to diverge from the true density slope, in particular for lower-mass galaxies with $\sigma_{e / 2} \lesssim 250 \mathrm{~km} \mathrm{~s}^{-1}$ (see Sect. 6.1.3). These model assmuptions could have also introduced biased mass dependence and biased redshift evolution as probed by $\gamma_{0}^{\mathrm{LD}}$ (see Sect. 6.4).

- We compared slope $\gamma_{0}^{\mathrm{LD}}$ to several different slope estimators $\left(\gamma^{\mathrm{MW}}, \gamma^{\mathrm{PL}}\right.$ and $\left.\gamma^{\mathrm{AV}}\right)$, which measure some intrinsic slopes of the central density profiles. In particular, the power-law fitted slope $\gamma^{\mathrm{PL}}\left(R_{\text {eff }}\right)$ decreases with increasing $\sigma_{e / 2}$ and is on average (slightly) steeper than isothermal, consistent with the observational results from 2-D kinematical data (e.g., Barnabè et al. 2011; Cappellari et al. 2015). In comparison, both $\gamma_{\text {tot }}^{\mathrm{MW}}\left(R_{\mathrm{eff}}\right)$ and $\gamma_{0}^{\mathrm{LD}}$ are shallower than isothermal; and $\gamma_{\text {tot }}^{\mathrm{MW}}\left(R_{\mathrm{eff}}\right)>\gamma_{0}^{\mathrm{LD}}$ was observed especially for lower-mass galaxies $\left(\sigma_{e / 2} \lesssim 250 \mathrm{~km} \mathrm{~s}^{-1}\right)$. The difference is due to a combination of two effects, which hold for the majority of the selected galaxy samples: (1) the power-law assumption breaks down and, in particular, the curvature parameter exceeds unity, i.e., $\xi_{\mathrm{M}_{3 \mathrm{~d}}}>1.0$, essentially resulting in steeper (local) density slopes at larger radii; and (2) on average, the effective radii $R_{\text {eff }}$ are larger than the Einstein radii $R_{\mathrm{E}}$, which eventually makes $\gamma^{\mathrm{MW}}\left(R_{\text {eff }}\right)$ larger than $\gamma_{0}^{\mathrm{LD}}$, as the latter quantity is essentially normalized at $R_{\mathrm{E}}$ (see Sect.6.2).

- The baryonic component is much more centrally concentrated than the total matter distribution. Due to the existence of baryons, the inner dark matter slope is notably steeper than the NFW prediction (see Sect.6.3). For the selected early-type galaxy samples from the simulation, the density slopes, either of the individual dark matter and baryonic components, or of the sum of the two, become shallower with cosmic time (see Sect.6.4).

- Several disagreements between the simulation and observational results that we found in this work seem to be related: the simulation predicted some higher central dark matter fractions, which would have suppressed the dominanting role of baryons and thus led to somehow shallower total density profiles in the inner regions of galaxies. The tension pose a potential challenge to the stellar formation and feedback models adopted by the simulation.

It is worth noting that for the selected early-type Illustris galaxies, we obtained self-consistent findings regarding the mass dependences and redshift evolutions of their colours, central cold gas (HI) fractions, stellar orbital anisotropies and central total density slopes. The early-type 
galaxies at higher redshifts are seen to be bluer and to contain a higher fraction of cold gas in their central regions. These features indicate star formation activity in their recent histories, which also leads to steeper central density profiles and more tangentially anisotropic stellar orbits. In comparison, their lower-redshift counterparts host much older stellar populations with redder colours and smaller cold gas fractions. In particular, as a consequence of another 7 Gyrs of passive evolution, their central density profiles become shallower and develop more radially anisotropic orbits. To test these theoretical predictions, 2-D kinematical data and high-resolution multi-band imaging data are required for a large number of galaxies, and out to high redshifts.

\section{ACKNOWLEDGEMENTS}

We would like to thank Stefan Hilbert, Hongyu Li, Yiping Shu, Adam Bolton and Matthias Bartelmann for various useful discussions. We would like to acknowledge the constructive discussion held at the TAP group meeting, in particular we thank Robert Grand, Ruediger Pakmor, Christine Simpson and Thomas Guillet. We would also like to thank the anonymous referee for very insightful and useful comments to improve the quality of the paper. DDX and VS would like to thank the Klaus Tschira Foundation. VS acknowledges support through the European Research Council under ERC-StG grant EXAGAL-308037. DS acknowledges funding support from a Back to Belgium grant from the Belgian Federal Science Policy (BELSPO). MV gratefully acknowledges support of the Alfred P. Sloan Foundation and the MIT RSC Reed fund. 


\section{APPENDIX A: SUMMARY OF THE CATALOGUE FIELDS}

We calculated a variety of properties (including galaxy types, sizes, morphologies, photometries, matter contents and distributions, and measurements on strong lensing and stellar kinematics) for all the Illustris galaxies that have stellar masses $M_{*} \gtrsim 10^{10} M_{\odot}$ at redshift $z=[0.1,0.2,0.3,0.4,0.5,0.6,0.7,0.8,0.9,1.0]$. Accordingly we defined artificial source redshifts at $z_{\mathrm{s}}=[0.5,0.6,0.7,0.9,2.0,2.0,2.0,2.0,2.0,2.0]$ in order to calculate the expected Einstein radii $R_{\mathrm{E}}$ for the galaxies. These choices of $z_{\mathrm{s}}$ were motived by the lens-source redshift distributions of the SLACS and SL2S surveys. All these data are publicly available at the Illustris website (www.illustris-project.org). In this Appendix, we give an overview of the catalogued quantities in Table A1 to Table A6 where we only provide descriptions of the projection-dependent quantities in their $\mathrm{X}$-projection (those for the $\mathrm{Y}$ - and Z-projections follow the same fashion) for the sake of simplicity.

In the catalogue, all radii involved are centred on the galactic centre of light (see Table. A3), which is also referred to as the "galaxy centre". All properties that were evaluated at a given radius were obtained through interpolation using the polynomial functions that were fitted to the corresponding radial distributions (assuming a circular/spherical symmetry)

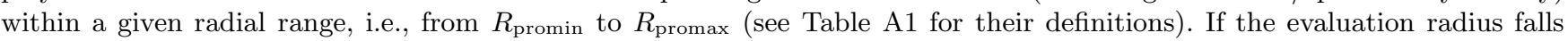
beyond this range, then the evaluation was actually made at the closer boundary radius of either $R_{\text {promin }}$ or $R_{\text {promax }}$. For density slopes that were measured between a given radial range of $\left(r_{1}, r_{2}\right)$, if $r_{2}<R_{\text {promin }}$ or $r_{1}>R_{\text {promax }}$ was true, then the slope was set to be the default value of 1E10. In addition, any fractional quantity should be a number between 0.0 and 1.0; however the interpolated value could go beyond this range if the interpolation radius is close to the boundaries where the polynomial fit becomes divergent. In this case, the interpolated fractions would be set to be the default value of -1.0. Tables given below further clarify any other specific cases where the default values would be reached.

We note that apart from the online catalogue, galaxy surface brightness distribution, as well as the best-fitted Sersic, de Vaucouleurs and exponential profiles (see Sect.2 for details) can be available by sending e-mail request to the author. In addition, the following radial profiles (and the polynomial fitting functions) of the projected/3d matter distribution that were extracted under the assumption of a circular/spherical symmetry (in regardless of galaxy types) within a radial range between $R_{\text {promin }}$ and $R_{\text {promax }}$ can be also available by sending e-mail request to the author. These profiles include: (1) the convergence (surface density) distribution $\kappa(R)$ at a given projected radius of $R,(2)$ the mean convergence distribution $\bar{\kappa}(\leqslant R) \equiv 2 \int_{0}^{R} R^{\prime} \kappa\left(R^{\prime}\right) \mathrm{d} R^{\prime} / R^{2}$, (3) the projected local dark matter fraction $f_{\mathrm{dm}}^{2 D}(R)$, (4) the projected cumulative dark matter fraction $\overline{f_{\mathrm{dm}}^{2 D}}(\leqslant R)$, (5) the total matter density distribution $\rho(r)$ at a 3D radius of $r,(6)$ the local dark matter fraction $f_{\mathrm{dm}}^{3 D}(r),(7)$ the cumulative dark matter fraction $\overline{f_{\mathrm{dm}}^{3 D}}(\leqslant r),(8)$ the cumulative gas fraction $\overline{f_{\text {gas }}^{3 D}}(\leqslant r)$, (9) the cumulative cold gas $(\mathrm{HI})$ fraction $\overline{f_{\mathrm{cgs}}^{3 D}}(\leqslant r)$. Note that all the mass fraction distributions are with respect to the total matter distribution. 
Table A1. Galaxy size measurements. Note that all quantities below are calculated from the galactic centre of light (see Table.A3) and are in unit of arcsec. For more details, see Sect. 2.3 and Sect. 2.4.

\begin{tabular}{|c|c|}
\hline Group Name & Description \\
\hline R_promin & $\begin{array}{l}\text { The minimum of the radial range, within which radial distributions of relevant quantities were measured } \\
\text { (and used for interpolation); set to be the angular size (at the snapshot redshift) corresponding to a physical } \\
\text { scale of } 0.7 \mathrm{kpc} \text {, which is the softening length of the simulation }\end{array}$ \\
\hline R_promax & $\begin{array}{l}\text { The maximum of the radial range, within which radial distributions of relevant quantities were measured (and } \\
\text { used for interpolation); set to be the angular size (at the snapshot redshift) corresponding to } \min (5 \times \mathrm{hsmr}, 30 \mathrm{kpc}) \text {, } \\
\text { where hsmr is the half-stellar-mass radius of the galaxy subhalo as calculated by SUBFIND }\end{array}$ \\
\hline Rein_x & The Einstein radius in X-projection; set to be 0.0 if Rein_x $<$ R_promin \\
\hline Rc50_x & $\begin{array}{l}\text { The radius within which the projected cumulative dark matter fraction is } 50 \% \text { in X-projection; } \\
\text { set to be } 0.0 \text { if Rc50_x }<\text { R_promin; or set to be } 1 \mathrm{E} 10 \text { if Rc50_x }>\text { R_promax }\end{array}$ \\
\hline R150_x & $\begin{array}{l}\text { The radius at which the projected local dark matter fraction is } 50 \% \text { in X-projection; } \\
\text { set to be } 0.0 \text { if } \mathrm{R} 150 \_\mathrm{x}<\mathrm{R} \text {-promin; or set to be } 1 \mathrm{E} 10 \text { if } \mathrm{R} 150 \_\mathrm{x}>\mathrm{R} \_ \text {promax }\end{array}$ \\
\hline Reff_ser_x & The effective radius by fitting Sersic profile in rest-frame SDSS r-band in X-projection \\
\hline Reff_dev_x & The effective radius by fitting de Vaucouleurs profile in rest-frame SDSS r-band in X-projection \\
\hline Reff_exp_x & The effective radius by fitting exponential profile in rest-frame SDSS r-band in X-projection \\
\hline Reff_of_sdss_umod_x & The Sersic-fitted effective radius in observer-frame SDSS u-band in X-projection \\
\hline Reff_of_sdss_u_x & $\begin{array}{l}\text { The radius which encloses half of the total luminosity measured in observer-frame SDSS u-band within } \\
\text { a projected radius of } 30 \mathrm{kpc} \text { from galaxy centre in X-projection }\end{array}$ \\
\hline Reff_of_sdss_gmod_x & The Sersic-fitted effective radius in observer-frame SDSS g-band in X-projection \\
\hline Reff_of_sdss_g_x & $\begin{array}{l}\text { The radius which encloses half of the total luminosity measured in observer-frame SDSS g-band within } \\
\text { a projected radius of } 30 \mathrm{kpc} \text { from galaxy centre in X-projection }\end{array}$ \\
\hline Reff_of_sdss_rmod_x & The Sersic-fitted effective radius in observer-frame SDSS r-band in X-projection \\
\hline Reff_of_sdss_r_x & $\begin{array}{l}\text { The radius which encloses half of the total luminosity measured in observer-frame SDSS r-band within } \\
\text { a projected radius of } 30 \mathrm{kpc} \text { from galaxy centre in X-projection }\end{array}$ \\
\hline Reff_of_sdss_imod_x & The Sersic-fitted effective radius in observer-frame SDSS i-band in X-projection \\
\hline Reff_of_sdss_i_x & $\begin{array}{l}\text { The radius which encloses half of the total luminosity measured in observer-frame SDSS i-band within } \\
\text { a projected radius of } 30 \mathrm{kpc} \text { from galaxy centre in X-projection }\end{array}$ \\
\hline Reff_of_sdss_zmod_x & The Sersic-fitted effective radius in observer-frame SDSS z-band in X-projection \\
\hline Reff_of_sdss_z_x & $\begin{array}{l}\text { The radius which encloses half of the total luminosity measured in observer-frame SDSS z-band within } \\
\text { a projected radius of } 30 \mathrm{kpc} \text { from galaxy centre in X-projection }\end{array}$ \\
\hline Reff_rf_sdss_gmod_x & The Sersic-fitted effective radius in rest-frame SDSS g-band in X-projection \\
\hline Reff_rf_sdss_g_x & $\begin{array}{l}\text { The radius which encloses half of the total luminosity measured in rest-frame SDSS g-band within } \\
\text { a projected radius of } 30 \mathrm{kpc} \text { from galaxy centre in X-projection }\end{array}$ \\
\hline Reff_rf_sdss_rmod_x & The Sersic-fitted effective radius in rest-frame SDSS r-band in X-projection \\
\hline Reff_rf_sdss_r_x & $\begin{array}{l}\text { The radius which encloses half of the total luminosity measured in rest-frame SDSS r-band within } \\
\text { a projected radius of } 30 \mathrm{kpc} \text { from galaxy centre in X-projection }\end{array}$ \\
\hline Reff_rf_sdss_imod_x & The Sersic-fitted effective radius in rest-frame SDSS i-band in X-projection \\
\hline Reff_rf_sdss_i_x & $\begin{array}{l}\text { The radius which encloses half of the total luminosity measured in rest-frame SDSS i-band within } \\
\text { a projected radius of } 30 \mathrm{kpc} \text { from galaxy centre in X-projection }\end{array}$ \\
\hline Reff_of_hst_bmod_x & The Sersic-fitted effective radius in observer-frame HST B-F435w in X-projection \\
\hline Reff_of_hst_b_x & $\begin{array}{l}\text { The radius which encloses half of the total luminosity measured in observer-frame HST B-F435w within } \\
\text { a projected radius of } 30 \mathrm{kpc} \text { from galaxy centre in X-projection }\end{array}$ \\
\hline Reff_of_hst_vmod_x & The Sersic-fitted effective radius in observer-frame HST V-F606w in X-projection \\
\hline Reff_of_hst_v_x & $\begin{array}{l}\text { The radius which encloses half of the total luminosity measured in observer-frame HST V-F606w within } \\
\text { a projected radius of } 30 \mathrm{kpc} \text { from galaxy centre in X-projection }\end{array}$ \\
\hline Reff_of_hst_imod_x & The Sersic-fitted effective radius in observer-frame HST I-F814w in X-projection \\
\hline Reff_of_hst_i_x & $\begin{array}{l}\text { The radius which encloses half of the total luminosity measured in observer-frame HST I-F814w within } \\
\text { a projected radius of } 30 \mathrm{kpc} \text { from galaxy centre in X-projection }\end{array}$ \\
\hline Reff_rf_john_bmod_x & The Sersic-fitted effective radius in rest-frame Johnson B-band in X-projection \\
\hline Reff_rf_john_b_x & $\begin{array}{l}\text { The radius which encloses half of the total luminosity measured in rest-frame Johnson B-band within } \\
\text { a projected radius of } 30 \mathrm{kpc} \text { from galaxy centre in X-projection }\end{array}$ \\
\hline Reff_rf_john_vmod_x & The Sersic-fitted effective radius in rest-frame Johnson V-band in X-projection \\
\hline Reff_rf_john_v_x & $\begin{array}{l}\text { The radius which encloses half of the total luminosity measured in rest-frame Johnson V-band within } \\
\text { a projected radius of } 30 \mathrm{kpc} \text { from galaxy centre in X-projection }\end{array}$ \\
\hline
\end{tabular}


Table A2. Galaxy photometry. Note that all magnitudes below are absolute AB magnitudes. The surface brightnesses are in unit of mag $\operatorname{arcsec}^{-2}$. For more details, see Sect. 2.3 and Sect. 2.4 for details.

\begin{tabular}{|c|c|}
\hline Group Name & Description \\
\hline Sersic_m_x & The Sersic index of the best-fitted Sersic profile in rest-frame SDSS r-band in X-projection \\
\hline IRe_ser_x & The surface brightness at Reff_ser_x in rest-frame SDSS r-band in X-projection \\
\hline IRe_dev_x & The surface brightness at Reff_dev_x in rest-frame SDSS r-band in X-projection \\
\hline IRe_exp_x & The surface brightness at Reff_exp_x in rest-frame SDSS r-band in X-projection \\
\hline Mag_of_sdss_umod_x & The magnitude in observer-frame SDSS u-band derived from best-fitted Sersic model in X-projection \\
\hline Mag_of_sdss_u_x & $\begin{array}{l}\text { The magnitude in observer-frame SDSS u-band derived from direct measurement } \\
\text { within a projected radius of } 30 \mathrm{kpc} \text { from galaxy centre in X-projection }\end{array}$ \\
\hline Mag_of_sdss_gmod_x & The magnitude in observer-frame SDSS g-band derived from best-fitted Sersic model in X-projection \\
\hline Mag_of_sdss_g_x & $\begin{array}{l}\text { The magnitude in observer-frame SDSS g-band derived from direct measurement } \\
\text { within a projected radius of } 30 \mathrm{kpc} \text { from galaxy centre in X-projection }\end{array}$ \\
\hline Mag_of_sdss_rmod_x & The magnitude in observer-frame SDSS r-band derived from best-fitted Sersic model in X-projection \\
\hline Mag_of_sdss_r_x & $\begin{array}{l}\text { The magnitude in observer-frame SDSS r-band derived from direct measurement } \\
\text { within a projected radius of } 30 \mathrm{kpc} \text { from galaxy centre in X-projection }\end{array}$ \\
\hline Mag_of_sdss_imod_x & The magnitude in observer-frame SDSS i-band derived from best-fitted Sersic model in X-projection \\
\hline Mag_of_sdss_i_x & $\begin{array}{l}\text { The magnitude in observer-frame SDSS i-band derived from direct measurement } \\
\text { within a projected radius of } 30 \mathrm{kpc} \text { from galaxy centre in X-projection }\end{array}$ \\
\hline Mag_of_sdss_zmod_x & The magnitude in observer-frame SDSS z-band derived from best-fitted Sersic model in X-projection \\
\hline Mag_of_sdss_z_x & $\begin{array}{l}\text { The magnitude in observer-frame SDSS z-band derived from direct measurement } \\
\text { within a projected radius of } 30 \mathrm{kpc} \text { from galaxy centre in X-projection }\end{array}$ \\
\hline Mag_rf_sdss_gmod_x & The magnitude in rest-frame SDSS g-band derived from best-fitted Sersic model in X-projection \\
\hline Mag_rf_sdss_g_x & $\begin{array}{l}\text { The magnitude in rest-frame SDSS g-band derived from direct measurement } \\
\text { within a projected radius of } 30 \mathrm{kpc} \text { from galaxy centre in X-projection }\end{array}$ \\
\hline Mag_rf_sdss_rmod_x & The magnitude in rest-frame SDSS r-band derived from best-fitted Sersic model in X-projection \\
\hline Mag_rf_sdss_r_x & $\begin{array}{l}\text { The magnitude in rest-frame SDSS r-band derived from direct measurement } \\
\text { within a projected radius of } 30 \mathrm{kpc} \text { from galaxy centre in X-projection }\end{array}$ \\
\hline Mag_rf_sdss_imod_x & The magnitude in rest-frame SDSS i-band derived from best-fitted Sersic model in X-project \\
\hline Mag_rf_sdss_i_x & $\begin{array}{l}\text { The magnitude in rest-frame SDSS i-band derived from direct measurement } \\
\text { within a projected radius of } 30 \mathrm{kpc} \text { from galaxy centre in X-projection }\end{array}$ \\
\hline Mag_of_hst_bmod_x & The magnitude in observer-frame HST B-F435w derived from best-fitted Sersic model in X-projection \\
\hline Mag_of_hst_b_x & $\begin{array}{l}\text { The magnitude in observer-frame HST B-F435w derived from direct measurement } \\
\text { within a projected radius of } 30 \mathrm{kpc} \text { from galaxy centre in X-projection }\end{array}$ \\
\hline Mag_of_hst_vmod_x & The magnitude in observer-frame HST V-F606w derived from best-fitted Sersic model in X-pr \\
\hline Mag_of_hst_v_x & $\begin{array}{l}\text { The magnitude in observer-frame HST V-F606w derived from direct measurement } \\
\text { within a projected radius of } 30 \mathrm{kpc} \text { from galaxy centre in X-projection }\end{array}$ \\
\hline Mag_of_hst_imod_x & The magnitude in observer-frame HST I-F814w derived from best-fitted Sersic model in X-projection \\
\hline Mag_of_hst_i_x & $\begin{array}{l}\text { The magnitude in observer-frame HST I-F814w derived from direct measurement } \\
\text { within a projected radius of } 30 \mathrm{kpc} \text { from galaxy centre in X-projection }\end{array}$ \\
\hline Mag_rf_john_bmod_x & The magnitude in rest-frame Johnson B-band derived from best-fitted Sersic model in X-pro \\
\hline Mag_rf_john_b_x & $\begin{array}{l}\text { The magnitude in rest-frame Johnson B-band derived from direct measurement } \\
\text { within a projected radius of } 30 \mathrm{kpc} \text { from galaxy centre in X-projection }\end{array}$ \\
\hline Mag_rf_john_vmod_x & The magnitude in rest-frame Johnson V-band derived from best-fitted Sersic model in X-pro \\
\hline Mag_rf_john_v_x & $\begin{array}{l}\text { The magnitude in rest-frame Johnson V-band derived from direct measurement } \\
\text { within a projected radius of } 30 \mathrm{kpc} \text { from galaxy centre in X-projection }\end{array}$ \\
\hline MagB_ep5_x & $\begin{array}{l}\text { The rest-frame Johnson-B magnitude measured within a projected radius of } 0.5 \times \text { Reff_rf_john_bmod_x } \\
\text { from galaxy centre in X-projection }\end{array}$ \\
\hline MagB_e1_x & $\begin{array}{l}\text { The rest-frame Johnson-B magnitude measured within a projected radius of Reff_rf_john_bmod_x from } \\
\text { galaxy centre in X-projection }\end{array}$ \\
\hline MagB_e2_x & $\begin{array}{l}\text { The rest-frame Johnson-B magnitude measured within a projected radius of } 2.0 \times \text { Reff_rf_john_bmod_x } \\
\text { from galaxy centre in X-projection }\end{array}$ \\
\hline MagV_ep5_x & $\begin{array}{l}\text { The rest-frame Johnson-V magnitude measured within a projected radius of } 0.5 \times \text { Reff_rf_john_vmod_x } \\
\text { from galaxy centre in X-projection }\end{array}$ \\
\hline MagV_e1_x & $\begin{array}{l}\text { The rest-frame Johnson-V magnitude measured within a projected radius of Reff_rf_john_vmod_x from } \\
\text { galaxy centre in X-projection }\end{array}$ \\
\hline MagV_e2_x & $\begin{array}{l}\text { The rest-frame Johnson-V magnitude measured within a projected radius of } 2.0 \times \text { Reff_rf_john_vmod_x } \\
\text { from galaxy centre in X-projection }\end{array}$ \\
\hline
\end{tabular}




\section{$24 X u$ et al.}

Table A3. Galaxy morphologies. For more details, see Sect. 2.2.

\begin{tabular}{|c|c|}
\hline Group Name & Description \\
\hline Galxc_x & $\begin{array}{l}\text { The } \mathrm{x} \text {-coordinate (in the plane of projection) of the light centre in the rest-frame Johnson-V band in X-projection, } \\
\text { measured within a projected radius of } 3.0 \times \mathrm{hsmr} \text { from and with respect to the centre of subhalo as calculated by SUBFIND }\end{array}$ \\
\hline Galyc_x & $\begin{array}{l}\text { The y-coordinate (in the plane of projection) of the light centre in the rest-frame Johnson-V band in X-projection, } \\
\text { measured within a projected radius of } 3.0 \times \mathrm{hsmr} \text { from and with respect to the centre of subhalo as calculated by SUBFIND }\end{array}$ \\
\hline Galb2a_ep5_x & $\begin{array}{l}\text { The axial ratio of the projected (rest-frame Johnson- } \mathrm{V} \text { band) light distribution measured within } \\
\text { a radius of } 0.5 \times \text { Reff_rf_john_vmod_x from galaxy centre in X-projection }\end{array}$ \\
\hline Galb2a_e1_x & $\begin{array}{l}\text { The axial ratio of the projected (rest-frame Johnson-V band) light distribution measured within } \\
\text { a radius of Reff_rf_john_vmod_x from galaxy centre in X-projection }\end{array}$ \\
\hline Galb2a_e2_x & $\begin{array}{l}\text { The axial ratio of the projected (rest-frame Johnson-V band) light distribution measured within } \\
\text { a radius of } 2.0 \times \text { Reff_rf_john_vmod_x from galaxy centre in X-projection }\end{array}$ \\
\hline Subb2a_ep5_x & $\begin{array}{l}\text { The axial ratio of the projected total matter distribution measured within a radius of } \\
0.5 \times \text { Reff_rf_john_vmod_x from galaxy centre in X-projection }\end{array}$ \\
\hline Subb2a_e1_x & $\begin{array}{l}\text { The axial ratio of the projected total matter distribution measured within a radius of } \\
\text { Reff_rf_john_vmod_x from galaxy centre in X-projection }\end{array}$ \\
\hline Subb2a_e2_x & $\begin{array}{l}\text { The axial ratio of the projected total matter distribution measured within a radius of } \\
2.0 \times \text { Reff_rf_john_vmod_x from galaxy centre in X-projection }\end{array}$ \\
\hline GalRA_ep5_x & $\begin{array}{l}\text { The orientation angle of the projected (rest-frame Johnson- } \mathrm{V} \text { band) light distribution measured } \\
\text { within a radius of } 0.5 \times \text { Reff_rf_john_vmod_x from galaxy centre in X-projection }\end{array}$ \\
\hline GalRA_e1_x & $\begin{array}{l}\text { The orientation angle of the projected (rest-frame Johnson-V band) light distribution measured } \\
\text { within a radius of Reff_rf_john_vmod_x from galaxy centre in X-projection }\end{array}$ \\
\hline GalRA_e2_x & $\begin{array}{l}\text { The orientation angle of the projected (rest-frame Johnson- } \mathrm{V} \text { band) light distribution measured } \\
\text { within a radius of } 2.0 \times \text { Reff_rf_john_vmod_x from galaxy centre in X-projection }\end{array}$ \\
\hline SubRA_ep5_x & $\begin{array}{l}\text { The orientation angle of the projected total matter distribution measured within a radius of } \\
0.5 \times \text { Reff_rf_john_vmod_x from galaxy centre in X-projection }\end{array}$ \\
\hline SubRA_e1_x & $\begin{array}{l}\text { The orientation angle of the projected total matter distribution measured within a radius of } \\
\text { Reff_rf_john_vmod_x from galaxy centre in X-projection }\end{array}$ \\
\hline SubRA_e2_x & $\begin{array}{l}\text { The orientation angle of the projected total matter distribution measured within a radius of } \\
2.0 \times \text { Reff_rf_john_vmod_x from galaxy centre in X-projection }\end{array}$ \\
\hline TypeDec_x & Galaxy Type in X-projection: 1 for early-type; 0 for late-type; -1 if lack of resolution for surface brightness fitting \\
\hline
\end{tabular}


Table A4. Matter contents and fractions. Note that all masses below are in unit of $h^{-1} M_{\odot}$; all fractions are with respect to the total matter. To derive the percentage of HI out of the total gas content, one needs to take, e.g., Fcgs3_e1_x/Fgas3_e1_x.

\begin{tabular}{|c|c|}
\hline Group Name & Description \\
\hline Mrein_x & The mass projected within a radius of Rein_x from galaxy centre in X-projection; set to be 0.0 if Rein_x $<$ R_promin \\
\hline MstarB_e1_x & The stellar mass projected within a radius of Reff_rf_john_bmod_x from galaxy centre in X-projection \\
\hline MstarV_ep5_x & The stellar mass projected within a radius of $0.5 \times$ Reff_rf_john_vmod_x from galaxy centre in X-projection \\
\hline MstarV_e1_x & The stellar mass projected within a radius of Reff_rf_john_vmod_x from galaxy centre in X-projection \\
\hline MstarV_e2_x & The stellar mass projected within a radius of $2.0 \times$ Reff_rf_john_vmod_x from galaxy centre in X-projection \\
\hline MtotB_e2_x & The total mass projected within a radius of $2.0 \times$ Reff_rf_john_bmod_x from galaxy centre in X-projection \\
\hline MtotV_ep5_x & The total mass projected within a radius of $0.5 \times$ Reff_rf_john_vmod_x from galaxy centre in X-projection \\
\hline MtotV_e1_x & The total mass projected within a radius of Reff_rf_john_vmod_x from galaxy centre in X-projection \\
\hline MtotV_e2_x & The total mass projected within a radius of $2.0 \times$ Reff_rf_john_vmod_x from galaxy centre in X-projection \\
\hline Fdm2in5kpc_x & $\begin{array}{l}\text { The cumulative dark matter fraction within a projected radius of } 5 \mathrm{kpc} \text { from galaxy centre in X-projection; } \\
\text { set to be }-1.0 \text { if the angular scale of } 5 \mathrm{kpc} \text { is larger than R_promax }\end{array}$ \\
\hline Fdm2_e1_x & $\begin{array}{l}\text { The cumulative dark matter fraction within a projected radius of Reff_rf_john_vmod_x } \\
\text { from galaxy centre in X-projection }\end{array}$ \\
\hline Fdm2_e2_x & $\begin{array}{l}\text { The cumulative dark matter fraction within a projected radius of } 2.0 \times \text { Reff_rf_john_vmod_x } \\
\text { from galaxy centre in X-projection }\end{array}$ \\
\hline Fdm3_ep5_x & The cumulative dark matter fraction within a 3D radius of $0.5 \times$ Reff_rf_john_vmod_x from galaxy centre \\
\hline Fdm3_e1_x & The cumulative dark matter fraction within a 3D radius of Reff_rf_john_vmod_x from galaxy centre \\
\hline Fdm3_e2_x & The cumulative dark matter fraction within a 3D radius of $2.0 \times$ Reff_rf_john_vmod_x from galaxy centre \\
\hline Fgas3_ep5_x & The cumulative gas fraction within a 3D radius of $0.5 \times$ Reff_rf_john_vmod_x from galaxy centre \\
\hline Fgas3_e1_x & The cumulative gas fraction within a 3D radius of Reff_rf_john_vmod_x from galaxy centre \\
\hline Fgas3_e2_x & The cumulative gas fraction within a 3D radius of $2.0 \times$ Reff_rf_john_vmod_x from galaxy centre \\
\hline Fcgs3_ep5_x & The cumulative cold gas (HI) fraction within a 3D radius of $0.5 \times$ Reff_rf_john_vmod_x from galaxy centre \\
\hline Fcgs3_e1_x & The cumulative cold gas (HI) fraction within a 3D radius of Reff_rf_john_vmod_x from galaxy centre \\
\hline
\end{tabular}


Table A5. Stellar kinematics measurements. Note that all velocities below are in unit of $\mathrm{km} \mathrm{s}^{-1}$ and with respect to the centre-of-mass velocity of its host dark matter subhalo.

\begin{tabular}{|c|c|}
\hline Group Name & Description \\
\hline Vmean_mav_x & $\begin{array}{l}\text { The stellar-mass-weighted stellar line-of-sight mean velocity measured within a projected radius } \\
\text { of } 1.5 \text { arcsec from galaxy centre in X-projection }\end{array}$ \\
\hline Vmean_lav_x & $\begin{array}{l}\text { The (rest-frame SDSS-r band) luminosity-weighted stellar line-of-sight mean velocity measured } \\
\text { within a projected radius of } 1.5 \text { arcsec from galaxy centre in X-projection }\end{array}$ \\
\hline Vmean_ep5_x & $\begin{array}{l}\text { The (rest-frame SDSS-r band) luminosity-weighted stellar line-of-sight mean velocity measured } \\
\text { within a projected radius of } 0.5 \times \text { Reff_rf_john_vmod_x from galaxy centre in X-projection }\end{array}$ \\
\hline Vmean_e1_x & $\begin{array}{l}\text { The (rest-frame SDSS-r band) luminosity-weighted stellar line-of-sight mean velocity measured } \\
\text { within a projected radius of Reff_rf_john_vmod_x from galaxy centre in X-projection }\end{array}$ \\
\hline Vmean_e2_x & $\begin{array}{l}\text { The (rest-frame SDSS-r band) luminosity-weighted stellar line-of-sight mean velocity measured } \\
\text { within a projected radius of } 2.0 \times \text { Reff_rf_john_vmod_x from galaxy centre in X-projection }\end{array}$ \\
\hline Vsigma_mav_x & $\begin{array}{l}\text { The stellar-mass-weighted stellar line-of-sight velocity dispersion measured within a projected } \\
\text { radius of } 1.5 \text { arcsec from galaxy centre in X-projection }\end{array}$ \\
\hline Vsigma_lav_x & $\begin{array}{l}\text { The (rest-frame SDSS-r band) luminosity-weighted stellar line-of-sight velocity dispersion measured } \\
\text { within a projected radius of } 1.5 \text { arcsec from galaxy centre in X-projection }\end{array}$ \\
\hline Vsigma_ep5_x & $\begin{array}{l}\text { The (rest-frame SDSS-r band) luminosity-weighted stellar line-of-sight velocity dispersion measured } \\
\text { within a projected radius of } 0.5 \times \text { Reff_rf_john_vmod_x from galaxy centre in X-projection }\end{array}$ \\
\hline Vsigma_e1_x & $\begin{array}{l}\text { The (rest-frame SDSS-r band) luminosity-weighted stellar line-of-sight velocity dispersion measured } \\
\text { within a projected radius of Reff_rf_john_vmod_x from galaxy centre in X-projection }\end{array}$ \\
\hline Vsigma_e2_x & $\begin{array}{l}\text { The (rest-frame SDSS-r band) luminosity-weighted stellar line-of-sight velocity dispersion measured } \\
\text { within a projected radius of } 2.0 \times \text { Reff_rf_john_vmod_x from galaxy centre in X-projection }\end{array}$ \\
\hline Beta_mav_x & $\begin{array}{l}\text { The stellar-mass-weighted stellar orbital anisotropy parameter measured within a 3D radius of } \\
1.5 \text { arcsec from galaxy centre }\end{array}$ \\
\hline Beta_lav_x & $\begin{array}{l}\text { The (rest-frame SDSS-r band) luminosity-weighted stellar orbital anisotropy parameter measured } \\
\text { within a 3D radius of } 1.5 \text { arcsec from galaxy centre }\end{array}$ \\
\hline Beta_ep5_x & $\begin{array}{l}\text { The (rest-frame SDSS-r band) luminosity-weighted stellar orbital anisotropy parameter measured } \\
\text { within a } 3 \text { D radius of } 0.5 \times \text { Reff_rf_john_vmod_x from galaxy centre }\end{array}$ \\
\hline$\overline{\text { Beta_e1_x }}$ & $\begin{array}{l}\text { The (rest-frame SDSS-r band) luminosity-weighted stellar orbital anisotropy parameter measured } \\
\text { within a 3D radius of Reff_rf_john_vmod_x from galaxy centre }\end{array}$ \\
\hline Beta_e2_x & $\begin{array}{l}\text { The (rest-frame SDSS-r band) luminosity-weighted stellar orbital anisotropy parameter measured } \\
\text { within a 3D radius of } 2.0 \times \text { Reff_rf_john_vmod_x from galaxy centre }\end{array}$ \\
\hline
\end{tabular}


Table A6. Matter density slopes. For more details, see Sect. 6 .

\begin{tabular}{|c|c|}
\hline Group Name & Description \\
\hline slpMWtot_ep5_x & $\begin{array}{l}\text { The mass-weighted total density slope calculated using Eq. } 1 \text { of Dutton \& Treu 2014, evaluated } \\
\text { at a radius of } 0.5 \times \text { Reff_rf_john_vmod_x from galaxy centre }\end{array}$ \\
\hline slpMWtot_e1_x & $\begin{array}{l}\text { The mass-weighted total density slope calculated using Eq. } 1 \text { of Dutton \& Treu 2014, evaluated } \\
\text { at a radius of Reff_rf_john_vmod_x from galaxy centre }\end{array}$ \\
\hline slpMWtot_e2_x & $\begin{array}{l}\text { The mass-weighted total density slope calculated using Eq. } 1 \text { of Dutton \& Treu 2014, evaluated } \\
\text { at a radius of } 2.0 \times \text { Reff_rf_john_vmod_x from galaxy centre }\end{array}$ \\
\hline slp3tot_ep5_x & $\begin{array}{l}\text { The average total density slope calculated using Eq. (15) of the paper, between } 0.2-0.5 \text { times } \\
\text { Reff_rf_john_vmod_x }\end{array}$ \\
\hline slp3tot_e1_x & $\begin{array}{l}\text { The average total density slope calculated using Eq. (15) of the paper, between } 0.5-1.0 \text { times } \\
\text { Reff_rf_john_vmod_x }\end{array}$ \\
\hline slp3tot_e2_x & $\begin{array}{l}\text { The average total density slope calculated using Eq. (15) of the paper, between } 0.5-2.0 \text { times } \\
\text { Reff_rf_john_vmod_x }\end{array}$ \\
\hline slp3totPLf_ep5_x & The fitted power-law slope of the total density distribution between $0.2-0.5$ times Reff_rf_john_vmod_x \\
\hline slp3totPLf_e1_x & The fitted power-law slope of the total density distribution between $0.5-1.0$ times Reff_rf_john_vmod_x \\
\hline slp3totPLf_e2_x & The fitted power-law slope of the total density distribution between $0.5-2.0$ times Reff_rf_john_vmod_x \\
\hline slpJESER_x & $\begin{array}{l}\text { The total density slope derived by combining strong lensing measurement of Mrein_x and single-aperture } \\
\text { stellar kinematics data of Vsigma_lav_x, assuming the stellar orbital anisotropy is given by Beta_lav_x; } \\
\text { set to be } 1 \text { E10 if Rein_x }<\text { R_promin }\end{array}$ \\
\hline slpJEbeta0_x & $\begin{array}{l}\text { The total density slope derived by combining strong lensing measurement of Mrein_x and } \\
\text { single-aperture stellar kinematics data of Vsigma_lav_x, assuming isotropic stellar orbital distribution; } \\
\text { set to be } 1 \text { E10 if Rein_x }<\text { R_promin }\end{array}$ \\
\hline slp3dm_ep5_x & $\begin{array}{l}\text { The average dark matter density slope calculated using Eq. (15) of the paper, between } 0.2-0.5 \\
\text { times Reff_rf_john_vmod_x }\end{array}$ \\
\hline slp3dm_e1_x & $\begin{array}{l}\text { The average dark matter density slope calculated using Eq. (15) of the paper, between } 0.5-1.0 \\
\text { times Reff_rf_john_vmod_x }\end{array}$ \\
\hline slp3dm_e2_x & $\begin{array}{l}\text { The average dark matter density slope calculated using Eq. (15) of the paper, between } 0.5-2.0 \\
\text { times Reff_rf_john_vmod_x }\end{array}$ \\
\hline slp3dmPLf_ep5_x & $\begin{array}{l}\text { The fitted power-law slope of the dark matter density distribution between } 0.2-0.5 \text { times } \\
\text { Reff_rf_john_vmod_x }\end{array}$ \\
\hline slp3dmPLf_e1_x & $\begin{array}{l}\text { The fitted power-law slope of the dark matter density distribution between } 0.5-1.0 \text { times } \\
\text { Reff_rf_john_vmod_x }\end{array}$ \\
\hline slp3dmPLf_e2_x & $\begin{array}{l}\text { The fitted power-law slope of the dark matter density distribution between } 0.5-2.0 \text { times } \\
\text { Reff_rf_john_vmod_x }\end{array}$ \\
\hline slp3st_ep5_x & $\begin{array}{l}\text { The average stellar density slope calculated using Eq. (15) of the paper, } \\
\text { between } 0.2-0.5 \text { times Reff_rf_john_vmod_x }\end{array}$ \\
\hline slp3st_e1_x & $\begin{array}{l}\text { The average stellar density slope calculated using Eq. (15) of the paper, } \\
\text { between } 0.5-1.0 \text { times Reff_rf_john_vmod_x }\end{array}$ \\
\hline slp3st_e2_x & $\begin{array}{l}\text { The average stellar density slope calculated using Eq. (15) of the paper, } \\
\text { between } 0.5-2.0 \text { times Reff_rf_john_vmod_x }\end{array}$ \\
\hline slp3stPLf_ep5_x & The fitted power-law slope of the stellar density distribution between $0.2-0.5$ times Reff_rf_john_vmod_x \\
\hline slp3stPLf_e1_x & The fitted power-law slope of the stellar density distribution between $0.5-1.0$ times Reff_rf_john_vmod_x \\
\hline slp3stPLf_e2_x & The fitted power-law slope of the stellar density distribution between $0.5-2.0$ times Reff_rf_john_vmod_x \\
\hline
\end{tabular}




\section{REFERENCES}

Auger M. W., Treu T., Bolton A. S., Gavazzi R., Koopmans L. V. E., Marshall P. J., Moustakas L. A., Burles S., 2010b, ApJ, 724, 511

Auger M. W., Treu T., Gavazzi R., Bolton A. S., Koopmans L. V. E., Marshall P. J., 2010a, ApJ Letters, 721, L163

Barnabè M., Czoske O., Koopmans L. V. E., Treu T., Bolton A. S., 2011, MNRAS, 415, 2215

Barnabè M., Czoske O., Koopmans L. V. E., Treu T., Bolton A. S., Gavazzi R., 2009, MNRAS, 399, 21

Bernardi M., Meert A., Sheth R. K., Vikram V., HuertasCompany M., Mei S., Shankar F., 2013, MNRAS, 436, 697

Bernardi M., Sheth R. K., Annis J., Burles S., Eisenstein D. J., Finkbeiner D. P., Hogg D. W., Lupton R. H., Schlegel D. J., SubbaRao M. e. a., 2003, AJ, 125, 1866

Binney J., Tremaine S., 2008, Galactic Dynamics: Second Edition. Princeton University Press

Bolton A. S., Brownstein J. R., Kochanek C. S., Shu Y., Schlegel D. J., Eisenstein D. J., Wake D. A., Connolly N., Maraston C., Arneson R. A., Weaver B. A., 2012, ApJ, 757,82

Bolton A. S., Burles S., Koopmans L. V. E., Treu T., Gavazzi R., Moustakas L. A., Wayth R., Schlegel D. J., 2008a, ApJ, 682, 964

Bolton A. S., Burles S., Treu T., Koopmans L. V. E., Moustakas L. A., 2007, ApJ Letters, 665, L105

Bolton A. S., Treu T., Koopmans L. V. E., Gavazzi R., Moustakas L. A., Burles S., Schlegel D. J., Wayth R., 2008b, ApJ, 684, 248

Brownstein J. R., Bolton A. S., Schlegel D. J., Eisenstein D. J., Kochanek C. S., Connolly N., Maraston C., Pandey P., Seitz S., Wake D. A., Wood-Vasey W. M., Brinkmann J., Schneider D. P., Weaver B. A., 2012, ApJ, 744, 41

Bruderer C., Read J. I., Coles J. P., Leier D., Falco E. E., Ferreras I., Saha P., 2016, MNRAS, 456, 870

Bruzual G., Charlot S., 2003, MNRAS, 344, 1000

Calzetti D., Kinney A. L., Storchi-Bergmann T., 1994, ApJ, 429, 582

Cappellari M., Romanowsky A. J., Brodie J. P., Forbes D. A., Strader J., Foster C., Kartha S. S., Pastorello N., Pota V., Spitler L. R., Usher C., Arnold J. A., 2015, ApJ Letters, 804, L21

Cappellari M., Scott N., Alatalo K., Blitz L., Bois M., Bournaud F., Bureau M., Crocker A. F., Davies R. L., Davis T. A. e. a., 2013, MNRAS, 432, 1709

Cardelli J. A., Clayton G. C., Mathis J. S., 1989, ApJ, 345, 245

Chabrier G., 2003, Astronomical Society of the Pacific, Publications, 115, 763

Ciotti L., Bertin G., 1999, A\&A, 352, 447

Cole S., Aragon-Salamanca A., Frenk C. S., Navarro J. F., Zepf S. E., 1994, MNRAS, 271, 781

Davis M., Efstathiou G., Frenk C. S., White S. D. M., 1985, ApJ, 292, 371

de Vaucouleurs G., 1948, Annales d'Astrophysique, 11, 247

Devriendt J. E. G., Guiderdoni B., 2000, A\&A, 363, 851

Devriendt J. E. G., Guiderdoni B., Sadat R., 1999, A\&A, 350,381

Djorgovski S., Davis M., 1987, ApJ, 313, 59

Dolag K., Borgani S., Murante G., Springel V., 2009, MN-
RAS, 399, 497

Dolag K., Komatsu E., Sunyaev R., 2015, ArXiv e-prints Dressler A., Lynden-Bell D., Burstein D., Davies R. L., Faber S. M., Terlevich R., Wegner G., 1987, ApJ, 313, 42 Dubois Y., Gavazzi R., Peirani S., Silk J., 2013, MNRAS, 433, 3297

Dutton A. A., Treu T., 2014, MNRAS, 438, 3594

Dye S., Negrello M., Hopwood R., Nightingale J. W., Bussmann R. S., Amber S., Bourne N., Cooray A., Dariush A., Dunne L., Eales S. A., Gonzalez-Nuevo J., Ibar E., Ivison R. J., Maddox S., Valiante E., Smith M., 2014, MNRAS, 440, 2013

Faber S. M., Dressler A., Davies R. L., Burstein D., Lynden-Bell D., 1987, in Faber S. M., ed., Nearly Normal Galaxies. From the Planck Time to the Present Global scaling relations for elliptical galaxies and implications for formation. pp 175-183

Faber S. M., Willmer C. N. A., Wolf C., Koo D. C., Weiner B. J., Newman J. A., Im M., Coil A. L., Conroy C., Cooper M. C. e. a., 2007, ApJ, 665, 265

Falco E. E., Gorenstein M. V., Shapiro I. I., 1985, ApJ Letters, 289, L1

Gavazzi R., Treu T., Marshall P. J., Brault F., Ruff A., 2012, ApJ, 761, 170

Gavazzi R., Treu T., Rhodes J. D., Koopmans L. V. E., Bolton A. S., Burles S., Massey R. J., Moustakas L. A., 2007, ApJ, 667, 176

Genel S., Fall S. M., Hernquist L., Vogelsberger M., Snyder G. F., Rodriguez-Gomez V., Sijacki D., Springel V., 2015, ApJ Letters, 804, L40

Genel S., Vogelsberger M., Springel V., Sijacki D., Nelson D., Snyder G., Rodriguez-Gomez V., Torrey P., Hernquist L., 2014, MNRAS, 445, 175

Graves G. J., Faber S. M., 2010, ApJ, 717, 803

Grillo C., 2010, ApJ, 722, 779

Grillo C., 2012, ApJ Letters, 747, L15

Grillo C., Gobat R., 2010, MNRAS, 402, L67

Guiderdoni B., Rocca-Volmerange B., 1987, A\&A, 186, 1

Hilbert S., Xu D., Schneider P., Springel V., Vogelsberger M., Hernquist L., 2016, ArXiv e-prints

Hinshaw G., Larson D., Komatsu E., Spergel D. N., Bennett C. L., Dunkley J., Nolta M. R., Halpern M., Hill R. S., Odegard N. e. a., 2013, ApJ Suppl., 208, 19

Hoekstra H., Yee H. K. C., Gladders M. D., 2004, ApJ, 606,67

Humphrey P. J., Buote D. A., 2010, MNRAS, 403, 2143

Humphrey P. J., Buote D. A., Gastaldello F., Zappacosta L., Bullock J. S., Brighenti F., Mathews W. G., 2006, ApJ, 646, 899

Hyde J. B., Bernardi M., 2009a, MNRAS, 394, 1978

Hyde J. B., Bernardi M., 2009b, MNRAS, 396, 1171

Johansson P. H., Naab T., Ostriker J. P., 2012, ApJ, 754, 115

Kauffmann G., 1996, MNRAS, 281, 487

Kauffmann G., White S. D. M., Guiderdoni B., 1993, MNRAS, 264, 201

Kitzbichler M. G., White S. D. M., 2007, MNRAS, 376, 2 Koopmans L. V. E., Bolton A., Treu T., Czoske O., Auger M. W., Barnabè M., Vegetti S., Gavazzi R., Moustakas L. A., Burles S., 2009, ApJ Letters, 703, L51

Koopmans L. V. E., Treu T., Bolton A. S., Burles S., Moustakas L. A., 2006, ApJ, 649, 599 
La Barbera F., de Carvalho R. R., 2009, ApJ Letters, 699, L76

Li H., Li R., Mao S., Xu D., Long R. J., Emsellem E., 2016, MNRAS, 455, 3680

Lin Y.-T., Mohr J. J., 2004, ApJ, 617, 879

MacArthur L. A., Courteau S., Holtzman J. A., 2003, ApJ, 582,689

Mandelbaum R., van de Ven G., Keeton C. R., 2009, MNRAS, 398, 635

Napolitano N. R., Romanowsky A. J., Tortora C., 2010, MNRAS, 405, 2351

Navarro J. F., Frenk C. S., White S. D. M., 1997, ApJ, 490, 493

Nelson D., Pillepich A., Genel S., Vogelsberger M., Springel V., Torrey P., Rodriguez-Gomez V., Sijacki D., Snyder G. F., Griffen B., Marinacci F., Blecha L., Sales L., Xu D., Hernquist L., 2015, ArXiv e-prints

Nipoti C., Treu T., Auger M. W., Bolton A. S., 2009b, ApJ Letters, 706, L86

Nipoti C., Treu T., Bolton A. S., 2009a, ApJ, 703, 1531

Oguri M., Rusu C. E., Falco E. E., 2014, MNRAS, 439, 2494

Puchwein E., Springel V., Sijacki D., Dolag K., 2010, MNRAS, 406, 936

Remus R.-S., Burkert A., Dolag K., Johansson P. H., Naab T., Oser L., Thomas J., 2013, ApJ, 766, 71

Remus R.-S., Dolag K., Naab T., Burkert A., Hirschmann M., Hoffmann T. L., Johansson P. H., 2016, ArXiv e-prints Romanowsky A. J., Kochanek C. S., 1999, ApJ, 516, 18

Rousseeuw P., Van Driessen K., 2006, Data Mining and Knowledge Discovery, 12, 29

Ruff A. J., Gavazzi R., Marshall P. J., Treu T., Auger M. W., Brault F., 2011, ApJ, 727, 96

Rusin D., Kochanek C. S., Keeton C. R., 2003, ApJ, 595, 29

Rusu C. E., Oguri M., Minowa Y., Iye M., Inada N., Oya S., Kayo I., Hayano Y., Hattori M., Saito Y., Ito M., Pyo T.S., Terada H., Takami H., Watanabe M., 2016, MNRAS, 458, 2

Salpeter E. E., 1955, ApJ, 121, 161

Schaye J., Crain R. A., Bower R. G., Furlong M., Schaller M., Theuns T., Dalla Vecchia C., Frenk C. S. e. a., 2015, MNRAS, 446, 521

Schneider P., Sluse D., 2013, A\&A, 559, A37

Sérsic J. L., 1963, Boletin de la Asociacion Argentina de Astronomia La Plata Argentina, 6, 41

Shu Y., Bolton A. S., Brownstein J. R., Montero-Dorta A. D., Koopmans L. V. E., Treu T., Gavazzi R., Auger M. W., Czoske O., Marshall P. J., Moustakas L. A., 2015, ApJ, 803, 71

Sijacki D., Vogelsberger M., Genel S., Springel V., Torrey P., Snyder G. F., Nelson D., Hernquist L., 2015, MNRAS, 452,575

Sluse D., Chantry V., Magain P., Courbin F., Meylan G., 2012, A\&A, 538, A99

Sonnenfeld A., Nipoti C., Treu T., 2014, ApJ, 786, 89

Sonnenfeld A., Treu T., Gavazzi R., Marshall P. J., Auger M. W., Suyu S. H., Koopmans L. V. E., Auger M. W., Suyu S. H., Koopmans L. V. E., Bolton A. S., 2012, ApJ, 752,163

Sonnenfeld A., Treu T., Gavazzi R., Suyu S. H., Marshall

P. J., Auger M. W., Nipoti C., 2013, ApJ, 777, 98
Sonnenfeld A., Treu T., Marshall P. J., Suyu S. H., Gavazzi R., Auger M. W., Nipoti C., 2015, ApJ, 800, 94

Spiniello C., Koopmans L. V. E., Trager S. C., Czoske O., Treu T., 2011, MNRAS, 417, 3000

Springel V., 2010, MNRAS, 401, 791

Springel V., White S. D. M., Tormen G., Kauffmann G., 2001, MNRAS, 328, 726

Tortora C., Napolitano N. R., Romanowsky A. J., Capaccioli M., Covone G., 2009, MNRAS, 396, 1132

Treu T., Auger M. W., Koopmans L. V. E., Gavazzi R., Marshall P. J., Bolton A. S., 2010, ApJ, 709, 1195

Treu T., Koopmans L. V. E., 2004, ApJ, 611, 739

Vogelsberger M., Genel S., Springel V., Torrey P., Sijacki D., Xu D., Snyder G., Bird S., Nelson D., Hernquist L., 2014a, Nature, 509, 177

Vogelsberger M., Genel S., Springel V., Torrey P., Sijacki D., Xu D., Snyder G., Nelson D., Hernquist L., 2014b, MNRAS, 444, 1518

Wellons S., Torrey P., Ma C.-P., Rodriguez-Gomez V., Pillepich A., Nelson D., Genel S., Vogelsberger M., Hernquist L., 2016, MNRAS, 456, 1030

Wellons S., Torrey P., Ma C.-P., Rodriguez-Gomez V., Vogelsberger M., Kriek M., van Dokkum P., Nelson E., Genel S., Pillepich A., Springel V., Sijacki D., Snyder G., Nelson D., Sales L., Hernquist L., 2015, MNRAS, 449, 361

White S. D. M., Rees M. J., 1978, MNRAS, 183, 341

$\mathrm{Xu}$ D., Sluse D., Schneider P., Springel V., Vogelsberger M., Nelson D., Hernquist L., 2016, MNRAS, 456, 739

Zibetti S., White S. D. M., Schneider D. P., Brinkmann J., 2005, MNRAS, 358, 949 